GMTSAR: An InSAR Processing System Based on Generic Mapping Tools

D. Sandwell, R. Mellors, X. Tong, M. Wei, P. Wessel

April 27, 2011 
This document was prepared as an account of work sponsored by an agency of the United States government. Neither the United States government nor Lawrence Livermore National Security, LLC, nor any of their employees makes any warranty, expressed or implied, or assumes any legal liability or responsibility for the accuracy, completeness, or usefulness of any information, apparatus, product, or process disclosed, or represents that its use would not infringe privately owned rights. Reference herein to any specific commercial product, process, or service by trade name, trademark, manufacturer, or otherwise does not necessarily constitute or imply its endorsement, recommendation, or favoring by the United States government or Lawrence Livermore National Security, LLC. The views and opinions of authors expressed herein do not necessarily state or reflect those of the United States government or Lawrence Livermore National Security, LLC, and shall not be used for advertising or product endorsement purposes.

This work performed under the auspices of the U.S. Department of Energy by Lawrence Livermore National Laboratory under Contract DE-AC52-07NA27344. 
Scripps Institution of Oceanography Technical Report

\section{GMTSAR: An InSAR Processing System Based on Generic Mapping Tools}

David Sandwell ${ }^{(1)}$, Rob Mellors $^{(2)}$, Xiaopeng Tong ${ }^{(1)}$, Matt Wei $^{(3)}$, and Paul Wessel ${ }^{(4)}$

May 1, 2011

(1) Scripps Institution of Oceanography, University of California, San Diego, La Jolla, CA

(2) Lawrence Livermore National Laboratory, Livermore, CA

(3) Woods Hole Oceanographic Institution, Woods Hole, MA

(4) Department of Geology and Geophysics, University of Hawaii at Manoa, HI 
Table of Contents:

Abstract

1. Introduction

1.1 Objectives and limitations of GMTSAR

1.2 Algorithms: SAR, InSAR and the need for precise orbits

1.2.1 Proper focus

1.2.2 Transformation from geographic to radar coordinates

1.2.3 Image alignment

1.2.4 Flattening interferogram - no trend removal

2. Software

2.1 Standard products

2.2 Software design

3. Processing Examples

3.1 Two-pass processing

3.2 Stacking and time series

3.3 ScanSAR Interferometry

4. References

5. Problems

Appendix A. Principles of Synthetic Aperture Radar

Appendix B. SAR Image Formation

Appendic C. InSAR

Appendix D. ScanSAR processor and interferometry

Appendix E. Geolocation accuracy for Pinon corner reflectors

Appendix F. Installation of GMTSAR 
Abstract

GMTSAR is an open source (GNU General Public License) InSAR processing system designed for users familiar with Generic Mapping Tools (GMT). The code is written in $\mathrm{C}$ and will compile on any computer where GMT and NETCDF are installed. The system has three main components: 1) a preprocessor for each satellite data type (e.g., ERS, Envisat, and ALOS) to convert the native format and orbital information into a generic format; 2) an InSAR processor to focus and align stacks of images, map topography into phase, and form the complex interferogram; 3) a postprocessor, mostly based on GMT, to filter the interferogram and construct interferometric products of phase, coherence, phase gradient, and line-of-sight displacement in both radar and geographic coordinates. GMT is used to display all the products as postscript files and kml-images for Google Earth. A set of C-shell scripts has been developed for standard 2pass processing as well as image alignment for stacking and time series. ScanSAR processing is also possible but requires a knowledgeable user. Users are welcome to contribute to this effort.

\section{Introduction}

GMTSAR differs from other InSAR systems such as ROI_PAC, Gamma, and DORIS because it relies on sub-meter orbital accuracy to greatly simplify the SAR and InSAR processing algorithms. Moreover large batches of SAR images can be automatically processed with no human intervention. The down side of this approach is that SAR satellites having less accurate orbits (> 10 m, e.g., RADARSAT-1 and JERS-1) cannot be easily processed using GMTSAR. Reliance on precise orbits also greatly simplifies the code so only 4 essential programs (esarp, xcorr, phasediff, conv) are needed beyond the GMT package. Moreover, geometric and phase accuracy ensures that mosaics of many frames along a long swath or combinations of ScanSAR subswaths will abut seamlessly in geographic coordinates. This document contains three main sections and 6 appendices. Section 1 is an introduction to GMTSAR and the geometric model used to create SAR and InSAR products without using ground control or applying orbital adjustments. Section 2 is a description of the overall software design as well as the standard output products. Section 3 provides recipes for the most common types of InSAR processing including 2-pass InSAR, stacking/time series, and ScanSAR interferometry. Appendix 
A is an overview of principles of SAR processing. Appendix B is a detailed description of the range-Doppler SAR processor and the parameter file. Appendix C provides the theory for InSAR processing on a spherical Earth with no approximations. Appendix D describes the algorithm that we use for ScanSAR interferometry. Appendix E provides data on the geolocation accuracy of ERS, Envisat, and ALOS SAR imagery. Appendix F provides instructions for installation and testing the GMTSAR package.

\subsection{Objectives and limitations of GMTSAR}

Creating synthetic aperture radar (SAR) images and interferograms (InSAR) from satellite radar measurements requires specialized front-end computer codes for focusing imagery and computing interferometric phase. However, much of the back-end processing and display can be accomplished with existing generic codes. We have developed a SAR/InSAR processing system that starts with raw SAR data in radar coordinates, along with precise orbital information, and computes interferometric products in geographic coordinates. Our design goals are to have a modular and portable system based on a minimum set of new software. The system relies on precise orbital information, as discussed below, and a consistent geometric model to achieve proper focus and image alignment. The SAR processor code was originally derived from the Stanford/JPL FORTRAN and re-written in the C programming language ensuring compilation on many platforms using the standard gcc compiler. The remainder of the front-end code has been developed and refined over the past decade as part of the SIOSAR and ALOS_preprocess packages.

For the back-end processing we use the Generic Mapping Tools (GMT), which is an open source collection of programs designed for manipulating and displaying geographic data sets [Wessel and Smith, 1998]. It is widely used among the geophysical community, well documented, and continuously updated. GMT includes efficient and robust programs for regridding, filtering, and trend removal, which makes it highly useful for some aspects of InSAR processing. GMT uses the NETCDF file format that allows the exchange of files among computers having different architectures.

The combined front- and back-end processing is done using the c-shell scripting language which is available on all UNIX platforms. We encourage the development of new modules and InSAR processing recipes from the broader InSAR community. The 
GMTSAR package also includes the capability for ScanSAR processing and is flexible enough for performing time series analysis on stacks of interferograms.

\subsection{Algorithms: SAR, InSAR, and the need for precise orbits}

Synthetic aperture radar (SAR) satellites collect swaths of side-looking radar echoes at a sufficiently high range resolution and along-track sampling rate to form high resolution imagery (Appendix A). The range resolution of the raw radar data is determined by the pulse length (or 1/bandwidth) and the incidence angle; a typical range resolution is $20 \mathrm{~m}$. The along-track or azimuth resolution of the outgoing microwave pulse is diffraction limited to an angle corresponding to the wavelength of the radar (e.g. $0.05 \mathrm{~m}$ ) divided by the length of the aperture (e.g. $10 \mathrm{~m})$. When this beam pattern is projected onto the surface of the earth at a range of say $850 \mathrm{~km}$, it illuminates $4250 \mathrm{~m}$ in the along-track dimension so the raw radar data are horribly out of focus in azimuth. The image can be focused on a point reflector on the ground by coherently summing thousands of consecutive echoes thus creating a synthetic aperture perhaps $4250 \mathrm{~m}$ long. Proper focus is achieved by summing the complex numbers along a constant range. The focused image contains both amplitude (backscatter) and phase (range) information for each pixel. The computer code to focus the raw radar echo data is called a SAR processor (esarp) and proper focus relies on having an accurate orbital trajectory for the satellite (Appendix B). For example the image will be out of focus if the range between the antenna and reflector has an error greater than about $1 / 4$ wavelength (e.g. $0.01 \mathrm{~m})$ over a distance of the synthetic aperture $(4250 \mathrm{~m})$.

A complex radar interferogram (InSAR) is created by multiplying the reference image by the complex conjugate of the repeat image (Appendix $C$ ). For the ideal case where the trajectories of the reference and repeat images are exactly coincident, the phase difference between the two images will reflect differences in range between the antenna and a target pixel. This could be due to line-of-sight motion of the target pixel or a travel time change due to the atmosphere or ionosphere. Normally there is an offset between the trajectories of the reference and repeat orbits. In this case the phase of the complex interferogram has an additional component due to the shape of the earth. The corrections for the curvature and topography of the earth are combined into one formula in the code phasediff (Appendix $\mathrm{C}$ ). This topographic phase can be removed from the interferogram 
if independent topography information is available [e.g., SRTM Farr et al., 2007]. An accurate orbit is needed to project the topography model from a latitude, longitude, and ellipsoidal height coordinate system into the range and azimuth coordinates of the radar image. An accurate orbit is also needed to correct the phase of the repeat image for the non-zero interferometric baseline since orbital error maps directly into interferometric phase.

The GMTSAR software will only provide reliable output for interferometric satellites where precise orbital information $(<1 \mathrm{~m}$ precision) is available. Today this includes (ERS-1, ERS-2, Envisat, ALOS, and TerraSAR-X). Note that RADARSAT-1 and JERS-1 cannot be easily processed with this software because their orbits are not accurate enough. The precise orbital information is used in 4 aspects of the InSAR processing discussed in the following text and is the key to robust and efficient software.

\subsubsection{Proper focus}

First proper focus of the SAR image involves the coherent summation of rangealigned echoes over the length of the synthetic aperture as shown in Figure 1.

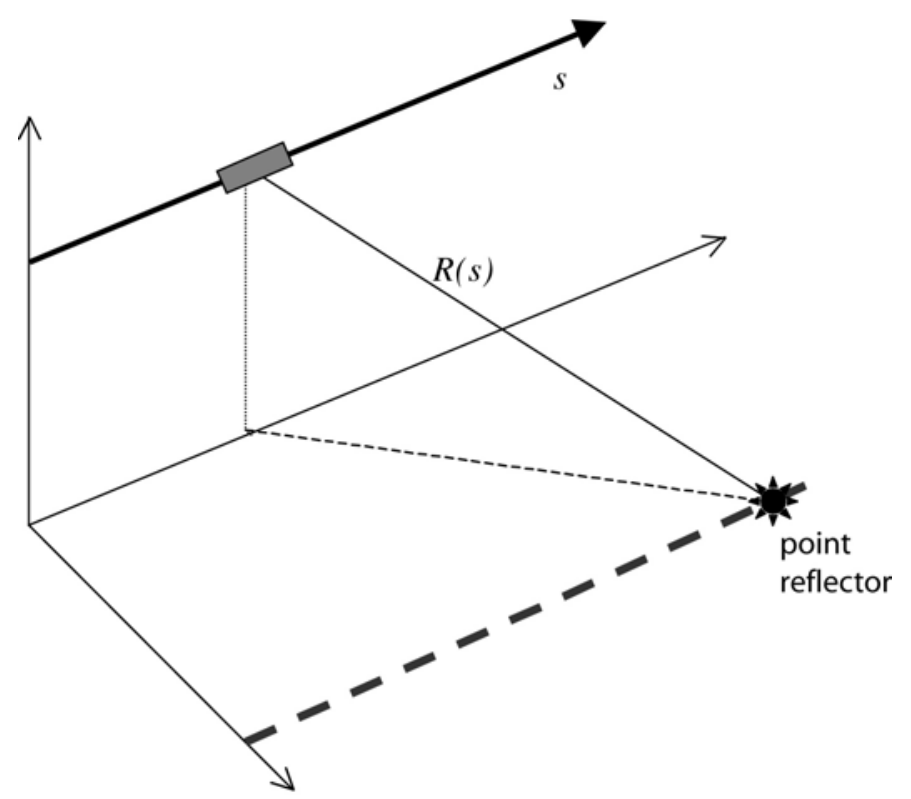

Figure 1. Geometry of SAR antenna flying over a point reflector on the ground. The range $R$ varies with slow time $s$ as measured by the precise orbit provided in an earth-fixed coordinate system. 
Therefore we need to calculate the range as a function of slow time $s$. First consider the case of a straight line orbit passing over a fixed point reflector. The range to the reflector would vary as a hyperbola with slow time. In the actual case the orbital path is elliptical and the earth is rotating so the range versus time function is a more complicated function. However, because the synthetic aperture is short $(<5 \mathrm{~km})$ compared with the nominal range from the satellite to the reflector $(\sim 800 \mathrm{~km})$, a 3-parameter parabolic approximation is commonly used to model range versus time [Curlander and McDonough, 1991; Appendix B]

$$
R(s)=R_{o}+\dot{R}_{o}\left(s-s_{o}\right)+\frac{\ddot{R}_{o}}{2}\left(s-s_{o}\right)^{2}+\ldots
$$

where $R_{o}$ is the closest approach of the spacecraft to the point reflector and $s_{o}$ is the time of closest approach. These three parameters $R_{o}, \dot{R}_{o}$, and $\ddot{R}_{o}$ are needed to focus an image (Appendix B). In terms of the SAR processor, these are called the near range $R_{o}$, the Doppler centroid $f_{D C}$ and the Doppler frequency rate $f_{R}$ which are related to the coefficients of this polynomial

$$
f_{D C}=\frac{-2 \dot{R}}{\lambda} \text { and } f_{R}=\frac{-2 \ddot{R}}{\lambda}
$$

where $\lambda$ is the wavelength of the radar. When a SAR image is focused at zero Doppler, the range rate at position $s_{o}$ is by definition zero.

\subsubsection{Transformation from geographic to radar coordinates}

The second use of precise orbital information is to map every point on the surface of the earth (lon, lat, topography) into the range and azimuth coordinates of the radar. This mapping provides a lookup table for transforming between geographic and radar coordinates. The algorithm for creating this mapping is conceptually simple but can be computationally expensive. The precise orbital information is used to determine the range from the satellite to a topography grid cell. The time of the minimum range is found using a Golden section search algorithm [Press et al., 1992]. A second order 
polynomial is fit to this range versus time function about the time of minimum range (equation (1)). If the SAR image is focused at zero Doppler then the range coordinate of the mapping is simply the minimum range and the azimuth coordinate is the time of the minimum range. Of course, by definition the range-rate, or Doppler, is zero at this azimuth position. If the radar image was focused at a Doppler centroid other than zero then equation (2) can be used to calculate the non-zero range-rate at this Doppler.

$\dot{R}=-\frac{\lambda f_{D C}}{2}$

Next by taking the derivative of the parabolic approximation one can determine the along-track time shift that will be produced by focusing at a non-zero Doppler. This is given by

$\Delta s=-\frac{\lambda f_{D C}}{2 \ddot{R}}$.

The corresponding range correction is given by

$\Delta R=\frac{\ddot{R}}{2} \Delta s^{2}$

This mapping from earth to radar coordinates described in equations 1-5 is perhaps the only really new aspect of GMTSAR and it allows a large simplification of the front-end processing code with respect to a more tolerant package such as ROI_PAC.

These two corrections are needed in the transformation from geographic to radar coordinates when the image is focussed at a Doppler other than zero. The accuracy of this approach where no ground control points are used has been determined using corner reflectors at Pinon Flat Observatory (Table 1). We have verified the accuracy of our approach using 27 ascending ALOS FBD/FBS images as well as 8 descending ScanSAR images (Table 2 and Appendix E). The results show a systematic range bias perhaps related to the ionosphere and troposphere delays (i.e., the dry troposphere should be -5.8 $\mathrm{m})$. The rms variation is typically $6 \mathrm{~m}$ in range and $4 \mathrm{~m}$ in azimuth. For the ScanSAR 
data the azimuth positions are 5 times less accurate due to the lower sampling density in each of the 5 subswaths. Since the highest resolution global topography data for the Earth has a pixel size of $30 \mathrm{~m}$, the mapping from lon, lat, topography into the range, azimuth, and topographic phase can be applied to non-zero baseline interferograms without any positional or phase adjustments. This provides a great simplification to the code because the topographic phase does not need to be spatially warped to match the master image of the interferogram. Although in some cases a range shift of up to one pixel is needed to account for the propagation delay through the atmosphere and ionosphere.

Table 1. Coordinates of Radar Reflectors

\begin{tabular}{|l|l|l|l|l|}
\hline \multicolumn{2}{|l|}{ position } & \multicolumn{2}{l|}{ orientation } \\
\hline lat & lon & height & elev. & azi. \\
\hline 33.612246 & -116.456768 & 1258.990 & $39^{\circ}$ & $257.5^{\circ}$ \\
\hline 33.612253 & -116.457893 & 1257.544 & $39^{\circ}$ & $102.5^{\circ}$ \\
\hline 33.607373 & -116.451836 & 1254.537 & $39^{\circ}$ & $102.5^{\circ}$ \\
\hline
\end{tabular}

Latitude and longitude in decimal degrees and height in meters relative to the WGS-84 coordinate system and ellipsoid. The survey point is the apex (lowest corner) of each reflector. There should be a correction for the offset between the phase center of the reflector and the apex.
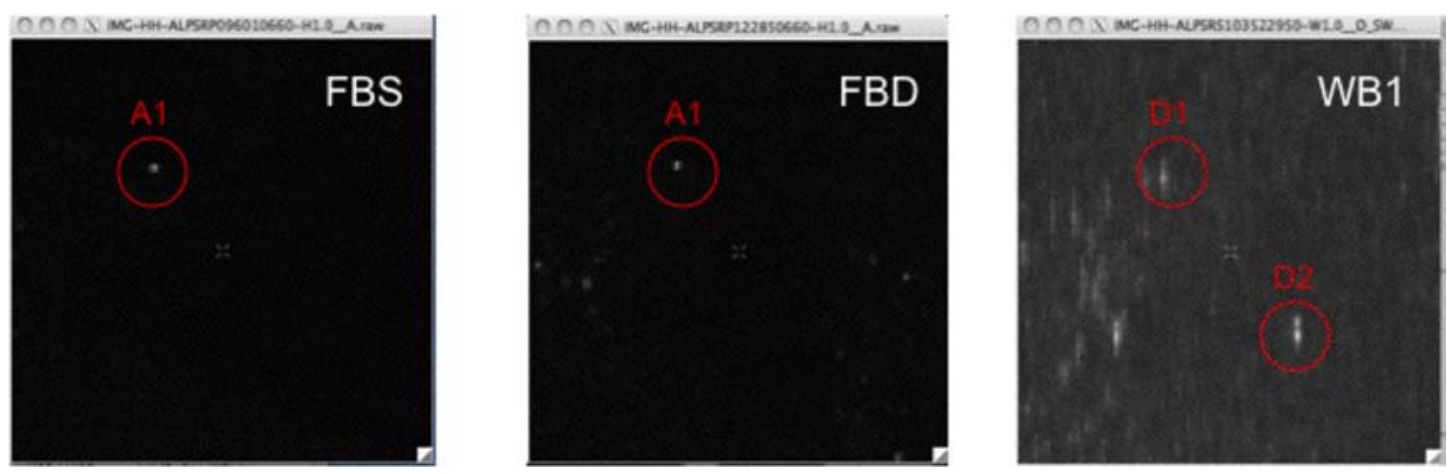

\begin{tabular}{|c|c|c|c|}
\hline & FBS & FBD & WB1 \\
\hline $\mathrm{N}$ & 18 & 9 & 8 \\
\hline ground-range & $-11.5 \pm 5.5 \mathrm{~m}$ & $-14.9 \pm 5.8 \mathrm{~m}$ & $-5.6 \pm 8.4 \mathrm{~m}$ \\
\hline azimuth & $1.3 \pm 3.7 \mathrm{~m}$ & $2.4 \pm 4.2 \mathrm{~m}$ & $-9.55 \pm 18.3 \mathrm{~m}$ \\
\hline
\end{tabular}

Table 2. Comparison between the position of radar corner reflectors predicted from ALOS orbital data and position seen in 35 focused SAR images. Three types of ALOS PALSAR data were used - fine beam single polarization (FBS), fine bean dual polarization (FBD) and 5-subswath ScanSAR (WB1). The mean and rms differences are consistent with all the error being due to propagation delays. The rms differences are consistent with point reflector resolution of the imagery. Orbital errors are probably less than 1 meter. 


\subsubsection{Image alignment}

The third use of the precise orbital information is to align the reference and repeat image to sub-pixel accuracy in order to form the interferogram. The corner reflector analysis above suggests that the geolocation based on the satellite orbit is only accurate to about 2 pixels in range and 1 pixel in azimuth so it is not accurate enough for subpixel image registration. Nevertheless this accuracy provides a very good starting point for a 2-dimensional image cross correlation algorithm (xcorr). Indeed our software xcorr, described below, uses a search window size of 64 pixels and has never failed to provide accurate co-registration even in cases where the interferometric coherence is close to zero.

\subsubsection{Flattening interferogram - no trend removal}

The fourth use of the precise orbit is for calculating the parallel and perpendicular components of the interferometric baseline that are needed for removing the interferometric phase for the range differences to every pixel in the image. This correction depends on both the orbital trajectory and the shape of the earth including the ellipsoidal, geoidal, and topographic components given in Appendix C. Another unique aspect of GMTSAR is that interferograms for long swaths of data can be calculated on a frame-by-frame basis and seamlessly reassembled in geographic co-ordinates. This is only possible because the baselines, orbital heights, and topographic data are all seamless at the frame boundaries. This seamless recombination of interferograms requires that the user select a common earth radius and near range for the entire swath in the file configure.txt. This feature is especially useful for ScanSAR-to-ScanSAR interferometry because each of the ScanSAR subswaths can be processed independently at their original azimuth sampling rate (i.e. PRF) and then re-assembled in geographic coordinates without adjustment. Frame-by-frame processing also has an advantage because the file size of one complex SAR image and its interferometric products can be far less than the 2 Gbyte file limit on 32-bit computers. Secondly, one can easily take advantage of multiple processors that are available on many computers to process multiple frames of interferometric products simultaneously, without having to rewrite any computer code. 


\section{Software}

\subsection{Standard Products}

Before introducing the main software components it is useful to describe the standard products created by the GMTSAR system using the script process 2 pass.csh.

name.PRM - Ascii file generated by the pre processing software. It contains all the information needed to focus and align a single look complex (SLC) image with a master image. This file contains all the important SAR and InSAR processing parameters and is more completely described in Appendix B.

name.raw - File contains the raw signal data. Each row of signal data has a 412 byte header record containing timing and other information followed by the radar echo stored as unsigned 2-byte complex numbers ( 1 byte real and 1 byte imaginary).

name.SLC - File contains the focused single look complex (SLC) image. The signed 4byte complex numbers are stored as unsigned short integers (2-bytes real and 2-bytes imaginary). The numbers are scaled to utilize the full dynamics range of a short integer. (An 8-byte complex output option is also provided by the SAR processor.)

trans.dat - File contains the mapping between range, azimuth coordinates and longitude, latitude, topography coordinates. The topography is calculated with respect to a local spherical approximation to the earth $($ Appendix $C)$ and therefore large negative and positive numbers are possible.

The format is: range azimuth topography longitude latitude $11301.58758718451 .999997-272.883315-115.15666732 .373333$ $11308.90945018451 .999997-273.413527-115.15611132 .373333$

topo_ra.grd - Netcdf format grid of range, azimuth, and topography.

real.grd or imag.grd - Netcdf grids of range, azimuth, and the real or imaginary part of the complex interferogram (files are deleted when no longer needed).

phase.grd - Netcdf grid of range, azimuth, and interferometric phase modulus $2 \pi$. corr.grd - Netcdf grid of range, azimuth, and interferometrric coherence (0-1.) xphase.grd - Netcdf grid of range, azimuth, and phase gradient in the range direction in units of radians per pixel (optional).

yphase.grd - Netcdf grid of range, azimuth, and phase gradient in the azimuth direction in units of radians per pixel (optional).

mask.grd - Netcdf grid of range, azimuth, and $\mathrm{NaN}$ or 1 . NaN signifies data should be masked.

unwrap.grd - Netcdf grid of unwrapped interferometric phase in radians (optional).

When the interferometric products have been transformed back to geographic coordinates the file names include an_ll. For example phase_ll.grd is a netcdf file of longitude, latitude, and interferometric phase modulus $2 \pi$. 
Files having a .ps suffix are postscript plots of the quantity. For example phase_mask_ll.ps is a postscript plot of masked phase in geographic coordinates.

Files having a. $\mathrm{kml}$ suffix are Google Earth images. For example phase_mask_ll.kml is a Google Earth image of masked phase in geographic coordinates.

(Note that the GMT command grd2xyz phase.grd $-Z f>$ phase will produce a flat file of floats with a size of $4 *$ nrow*ncol bytes)

\subsection{Software Design}

The overall design of the software is illustrated in Figure 2. There is a set of preprocessing code for each of the satellites - currently ERS-1, ERS-2, Envisat, and ALOS. Data, leader, and orbit files provided by the space agency are normally in CEOS format and are converted into generic *.raw and *.PRM files. The pre-processing code checks for missing lines in the CEOS data file and aligns the radar echoes on a common near range or sample window start time (SWST). The output is a raw file (*.raw) that is ready to be focused in the SAR processor. The relevant parameters are extracted from the various leader and orbit files and used to prepare a parameter file (*.PRM) as fully described in Appendix B. After selecting the master image, a user-supplied topography grid of the area (dem.grd) is projected into radar coordinates to create a transformation file (trans.dat).

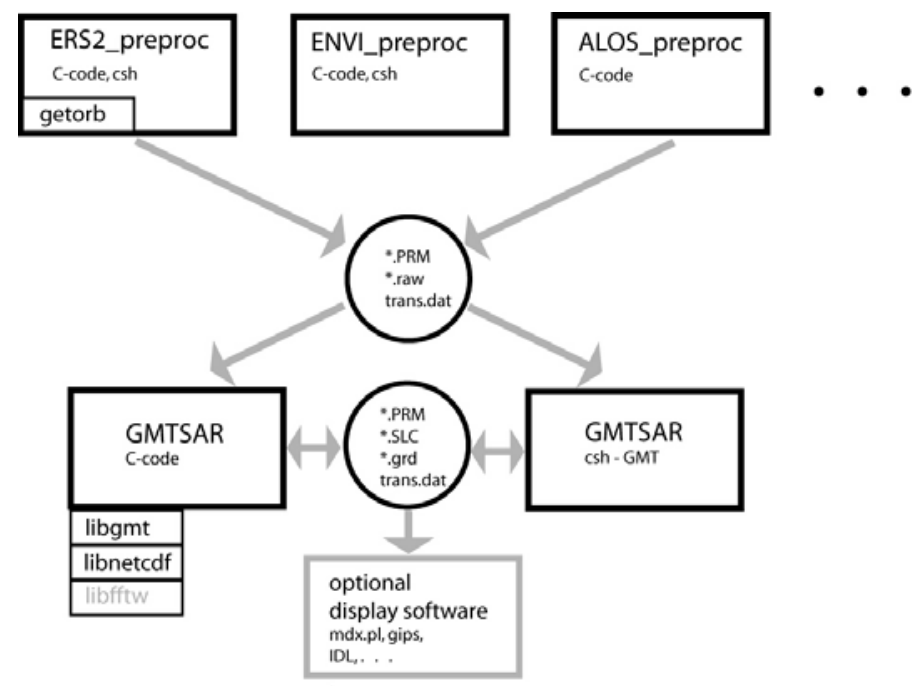

Figure 2. Design of GMTSAR and preprocessors.

The GMTSAR code is the same for all the satellite data types. There are 4 essential programs written in C called esarp, xcorr, phasediff, conv. The programs esarp and xcorr make extensive use of a 1-dimensional fast fourier transform (FFT) routine called 
cfft ld.c. There are three options for linking the cfftld.c code: the default option uses FFT routines from fftpack.c code [Swarztrauber, 1982] which is included in the GMTSAR distribution; the fastest option is to link with VECLIB routines included in the Mac OS X system; the third option is to use the fftw code that is distributed by http://www.fftw.org/. The optimized FFT codes will reduce the runtime of these programs by about $20-40 \%$. The programs phasediff, and conv read/write GMT grdfiles in netcdf format and therefore require that the user link with the libraries libgmt.a and libnetcdf.a. These programs, as well as many standard GMT routines, are called from shell scripts. A list of the current scripts is found by executing the command gmtsar.csh.

$\begin{array}{ll}\text { align.csh } & \text { - align a pair of SAR images } \\ \text { align_batch.csh } & \text { - align a stack of SAR images } \\ \text { baseline_table.csh } & \text { - make baseline vs time table } \\ \text { cleanup.csh } & \text { - cleanup the directories } \\ \text { dem2topo_ra.csh } & \text { - transform a dem into range and azimuth coordinates } \\ \text { filter.csh } & \text { - filter the interferogram and make amp, phase and corr } \\ \text { fitoffset.csh } & \text { - solve for the affine parameters } \\ \text { geocode.csh } & \text { - convert range/azimuth to lon/lat } \\ \text { grd2kml.csh } & \text { - make a kml file for google earth } \\ \text { intf.csh } & \text { - make the interferogram from a single pair of SLCs } \\ \text { intf_batch.csh } & \text { - make interferograms for a set of aligned SLCs } \\ \text { make_a_offset.csh } & \text { - make azimuth offsets } \\ \text { make_dem.csh } & \text { - construct a dem from tiles } \\ \text { pre_proc.csh } & \text { - preprocess the raw SAR data for a pair of images } \\ \text { pre_proc_batch.csh } & \text { - preprocess raw SAR data for a stack of images } \\ \text { p2p_ALOS.csh } & \text { - process an ALOS interferogram from end-to-end } \\ \text { p2p_ENVI.csh } & \text { - process an Envisat interferogram from end-to-end } \\ \text { p2p_ERS.csh } & \text { - process an ERS interferogram from end-to-end } \\ \text { proj_ll2ra.csh } & \text { - project a grd file from lon/lat to range/azimuth } \\ \text { proj_ll2ra_ascii.csh } & \text { - project points from lon/lat to range/azimuth } \\ \text { proj_ra2ll.csh } & \text { - project a grd file from range/azimuth to lon/lat } \\ \text { proj_ra2ll_ascii.csh } & \text { - project points from range/azimuth to lon/lat } \\ \text { sarp.csh } & \text { - focus a single SAR image } \\ \text { slc2amp.csh } & \text { - make a amplitude image from a SLC } \\ \text { snaphu.csh } & \text { - unwrap phase using snaphu } \\ \text { update_PRM.csh } & \text { - replace a value in a PRF-file } \\ & \\ \text { Executing one of the commands will provide its usage: } \\ \text { \%sarp.csh } & \\ \text { Usage: sarp.csh file.PRM } & \\ \text { Example: sarp.csh IMG-HH-ALPSRP049040660-H1.0_A.PRM }\end{array}$




\section{Processing Examples}

The modules of the GMTSAR package can be connected in a variety of ways to do specialized SAR and InSAR processing. The foundation of the code is all optimized, written in $\mathrm{C}$, and we hope is functionally stable. For example, the SAR processor esarp.c has remained stable for more than a decade. We expect this C-code as well as the GMT code, will be stable on a time frame of years. New preprocessor code will need to be written as new satellite data becomes available but everything downstream should not change significantly.

The flexible aspect of the package is the c-shell scripts to perform different types of InSAR processing. Indeed, c-shell is not a particularly good language for writing large scripts and we hope this part of the system will evolve based on input from knowledgeable users. So far we have developed three standard InSAR recipes. They are 2-pass processing, stacking for time series analysis, and ScanSAR to strip-mode interferometry. We will outline the processing stages in these three examples. The first example is also used for testing the installation (Appendix $F$ ) so we will step through the InSAR processing for a pair of ALOS SAR images spanning the 2010 M7.2 Baja, Mexico Earthquake.

\subsection{Two-pass processing}

A common approach to InSAR processing is to form an interferogram using two SAR images and a digital elevation model. A flow diagram of a script called process 2 pass.csh is shown in Figure 3. Selecting a pair of images having the desired perpendicular baseline and time span characteristics is an important issue that will be discussed in the next section under stacking and time series. This example recovers the coseismic displacement from an earthquake so the ALOS PALSAR images are selected as the pair spanning the M7.2 Baja, Mexico Earthquake having the minimum time span. To prepare for the processing one needs to generate a digital elevation grid of the area in longitude and latitude coordinates spanning an area greater than the SAR coverage (e.g., longitude -116.25 to -115.16 and latitude 32.16 to 33.16 ). The heights should be referenced to the WGS84 ellipsoid and not the geoid. The command make_dem.csh will make the grid but only if it has access to the tiles of SRTM or ASTER topography data as well as the EGM96 geoid model. A topographic grid of any land area can be created using the 
website http://topex.ucsd.edu/gmtsar. The user places this topography grid (dem.grd) in a directory called /topo and the two scenes of raw SAR data in L1.0 CEOS format in the directory called /raw. The first scene is the reference image and the second scene is the repeat image. (Later when discussing the alignment of large stacks of SAR images we will use additional terminology of a single master image and many slave images.) After modifying a file called configure.txt the user uses the command process2pass.csh IMG-HH-ALPSRP207600640-H1.0__A IMG-HH-ALPSRP227730640-H1.0__A configure.txt and waits for the results.

An overview of the 7 main processing steps is shown in Figure 3 and the modules are provided in Table 3. The first step is to preprocess raw SAR data and orbital information, usually in L1.0 CEOS format, to create an ascii parameter file (e.g. Table B1) and a raw data file (e.g., Figure B3). This preprocessing involves specialized code to extract orbital position and velocity information from the leader files, align the raw radar echoes on a common near range, and estimate the Doppler centroid of the raw data. The second step is to focus each image to create two single look complex images as described in Appendix B. The third step is to align the repeat image to the reference image. This is accomplished by first using the orbital information to estimate the shift in range an azimuth needed to align the upper left corner of the images. Then many small patches (e.g., 64x64) are extracted from each image and cross correlated to determine 6 affine parameters needed to warp the repeat image to match the second image. The repeat image is refocussed using these 6 parameters resulting in sub-pixel alignment between the reference and repeat images. Smaller scale pixel shifts due to large amplitude surface topography are corrected at the interfere step. The fourth step (dem2topo_ra) is to transform the digital elevation model from longitude, latitude, and topography into range, azimuth, and topography. This is done using the precise orbital information of the reference image. Sometimes a -1 pixel range shift (i.e., derived from a 


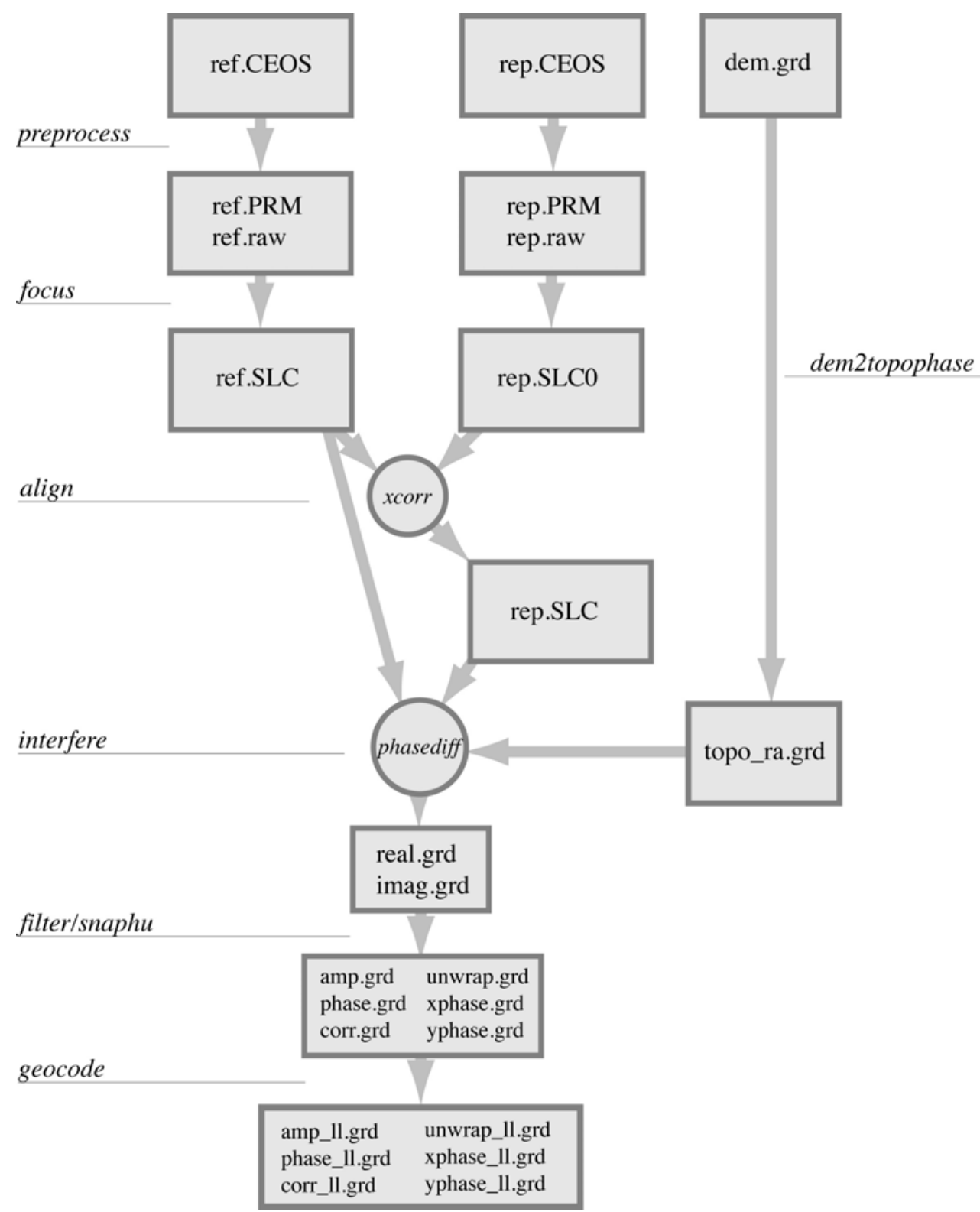

Figure 3. Flow diagram of 2-pass processing beginning with raw SAR and orbital data and a digital elevation grid (dem.grd) and ending with geocoded grids of interferometric products.

cross correlation between the amplitude image of the master and the range gradient of the topographic phase, offset_topo is needed to improve the alignment. The fifth step is to interfere the reference and repeat SLC's using the topo_ra.grd to both refine the image alignment of the repeat image due to topography parallax, and to remove the baselinedependent topographic phase from the repeat image prior to cross multiplication. Thus all the position and phase corrections are applied at the full resolution of the SLC's. The sixth step filter/snaphu is to low-pass filter the real and imaginary components of the 
interferogram and compute standard products of amplitude, phase, coherence. Optionally one can compute unwrapped phase using the snaphu program of Chen and Zebker [2002] as well as phase gradients [Sandwell and Price, 1998] in the range (xphase) and azimuth (yphase) directions. The final step is to geocode all the products by transforming from the range/azimuth coordinate system of the master image to longitude and latitude. The c-shell scripts also produce postscript images and kml-images of relevant products using the capabilities of GMT. An image of the sample interferogram superimposed on the dem is shown in Figure 4.

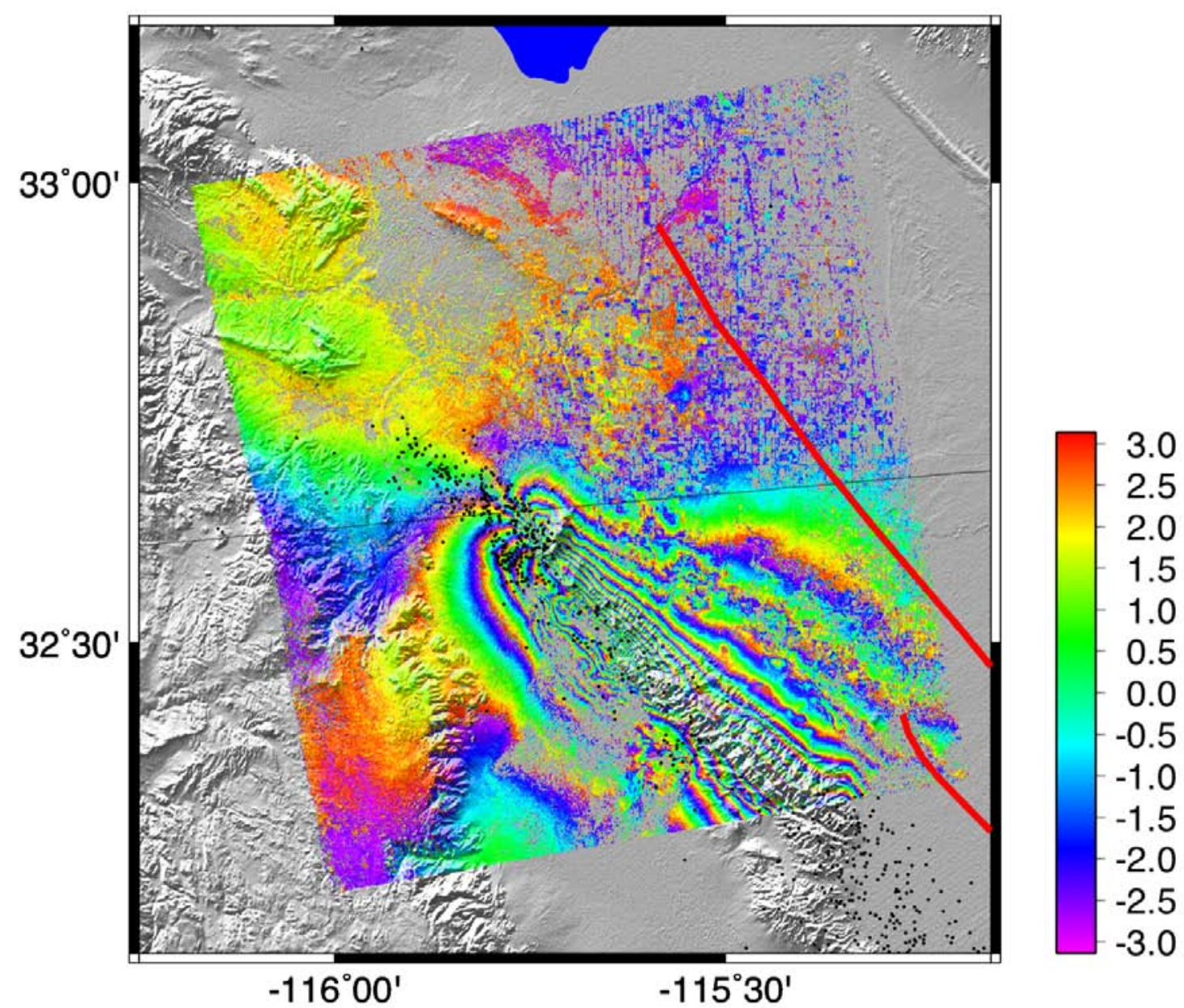

Figure 4. Interferometric phase for an ascending track (T211, F0640) of ALOS PALSAR data over the April 4, 2010, El Major-Cucapah earthquake, Baja Mexico. One fringe represents 11.8 $\mathrm{cm}$ displacement away from the radar. The phase is superimposed on the gradient of the topography and other components such as faults and earthquake epicenters are easily added. 
Table 3. Algorithm and codes for 2-pass processing - $p 2 p \_S A T . c s h$

\begin{tabular}{|c|c|c|}
\hline $\begin{array}{l}1 \text { - preprocess } \\
\text { pre_proc.csh }\end{array}$ & $\begin{array}{l}\text { update_PRM.csh } \\
\text { ALOS_pre_process.csh } \\
\text { ALOS_fbd2fbs.c } \\
\text { ALOS_fbs } 2 f b d . c\end{array}$ & \\
\hline $\begin{array}{l}2 \text { - align and focus } \\
\text { align.csh }\end{array}$ & $\begin{array}{l}\text { sarp.csh } \\
\text { ALOS_baseline.c } \\
\text { xcorr.c } \\
\text { fitoffset.csh } \\
\end{array}$ & $\begin{array}{l}\text { esarp.c } \\
\text { trend2d.c }\end{array}$ \\
\hline $\begin{array}{l}\text { 3- topo_ra } \\
\text { dem2topo_ra.csh }\end{array}$ & $\begin{array}{l}\text { grd2xyz.c } \\
\text { ALOS_llt2rat.c } \\
\text { blockmedian.c } \\
\text { surface.c } \\
\text { topo2phase.c } \\
\text { grdimage.c }\end{array}$ & \\
\hline $\begin{array}{l}4 \text {-interfere and filter } \\
\text { intf.csh } \\
\text { filter.csh }\end{array}$ & $\begin{array}{l}\text { ALOS_baseline.c } \\
\text { phasediff.c } \\
\text { conv.c } \\
\text { grdmath.c } \\
\text { grdimage.c } \\
\text { phasefilt.c }\end{array}$ & \\
\hline $\begin{array}{l}\text { 5- unwrap phase } \\
\text { snaphu.csh }\end{array}$ & $\begin{array}{l}\text { grdcut.c } \\
\text { grdmath.c } \\
\text { grd2xyz.c } \\
\text { snaphu.c } \\
\text { xyz2grd.c } \\
\text { grdimage.c }\end{array}$ & \\
\hline $\begin{array}{l}6 \text { - geocode } \\
\text { geocode.csh }\end{array}$ & $\begin{array}{l}\text { grdmath.c } \\
\text { proj_ra2ll.csh }\end{array}$ & $\begin{array}{l}\text { grd2xyz.c } \\
\text { gmtconvert.c } \\
\text { blockmedian.c } \\
\text { surface.c } \\
\text { grdtrack.c } \\
\\
\text { grdimage.c } \\
\text { ps2raster.c }\end{array}$ \\
\hline
\end{tabular}

GMTSAR commands are italic. black - c-shell script, red - GMTSAR C-code, blue - GMT C-code, green snaphu phase unwrapper [Chen and Zebker 2000].

Note this example if for processing an $A L O S$ interferogram. For another satellite (e.g. Envisat) replace the characters $A L O S$ by $E N V I$.

\subsection{Stacking for time series}

A second common approach to InSAR processing is to form a time series of deformation from a stack of images. There are a variety of methods ranging from averaging to small baseline subsets to time series to persistent scattering. The practical, and sometimes most challenging issue related to these approaches is to geometrically align a large stack of images and align this stack with a topographic phase model. A diagram of a stack of SAR images is shown in Figure 5. To achieve interferometric 
phase coherence, the alignment must have subpixel accuracy in the radar coordinates of range and azimuth. In addition, there should be greater than $20 \%$ overlap in the Doppler centroid or bursts in ScanSAR images. We use the terminology that many slave images are geometrically aligned with one master image. Once the alignment is done, an interferogram can be constructed from any pair of images in the stack. We use the terminology of reference and repeat images for the first and second acquisition of the interferometric pair, respectively.

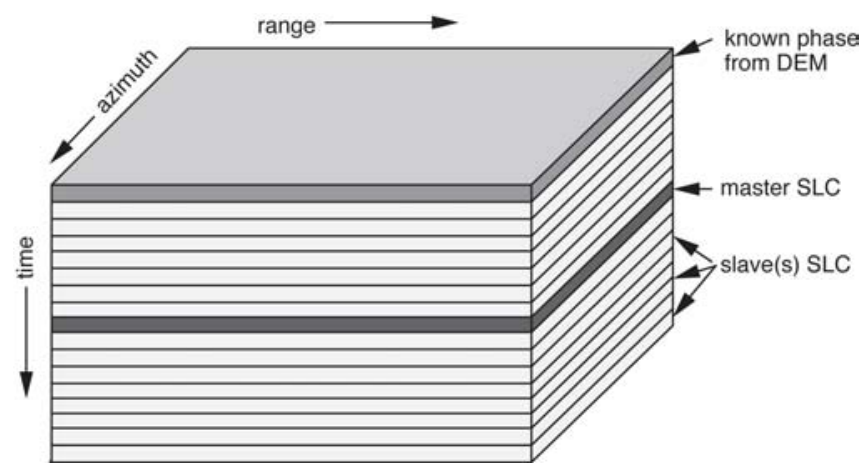

Figure 5. Schematic diagram of a stack of SAR images and a topographic phase image.

Image alignment is problematic if the perpendicular baseline between the master image and one of the slave images is greater than about 3/4 of the critical baseline because the images will be baseline decorrelated (Appendix $C$ ). Temporal decorrelation can also occur when the scattering surface of the earth changes between the master and slave acquisitions because of vegetation, snow, or other small-scale surface disturbances. As discussed above, our image alignment algorithm begins with a guess at the range and azimuth shift based on the precise orbit and then uses 2-dimensional cross correlation on small patches. If there are no areas in the images that are correlated then this cross correlation approach can fail to achieve subpixel correlation. A plot of perpendicular baseline versus time can be used to identify images that lie far from the master and therefore may not be suitable for subpixel alignment. An example of a baseline vs. time plot for an ALOS stack (Track 213, Frame 0660, Coachella Valley, CA) is shown in Figure 6. Temporal decorrelation is less of an issue in this desert area however, the baseline of ALOS drifts by more than $5 \mathrm{~km}$ over a time span of 2 years. The critical baseline for the lower bandwidth (FBD) data is $6 \mathrm{~km}$ so, for example the acquisition on orbit number 12285 lies more than $4 \mathrm{~km}$ from acquisition on orbit 13627. 


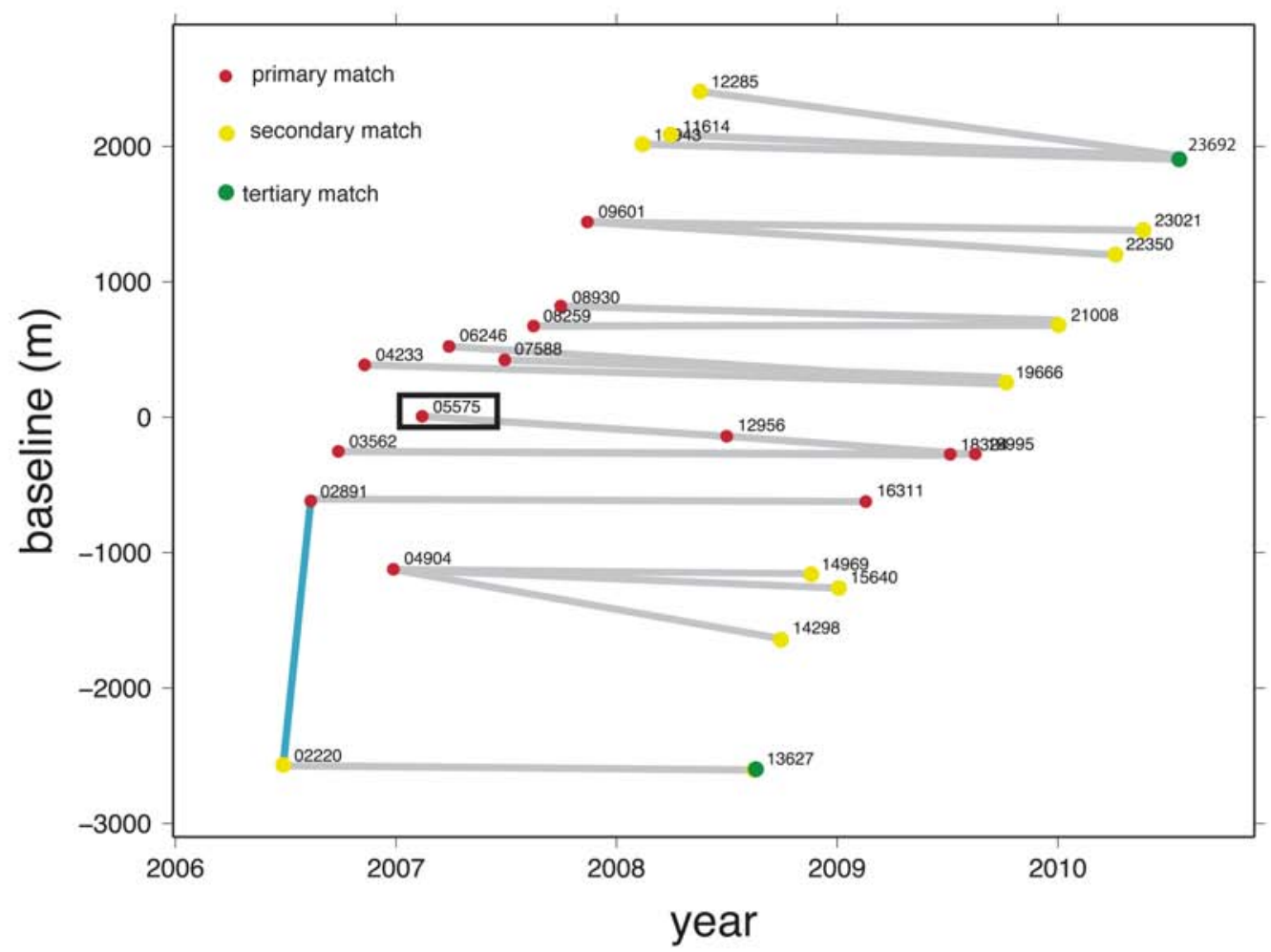

Figure 6 Baseline versus time plot partly created by the pre_proc_batch.csh command. Colored dots were added by user and represent the intended primary (red), secondary (yellow) and tertiary (green) image alignment. The grey lines were added and represent candidate interferograms. The interferometric pair marked by a blue line is used in Appendix $\mathrm{C}$ for assessing the accuracy of the earth curvature and topographic phase corrections.

We perform the image alignment using the following steps:

1) Preprocess all the images independently and examine the PRM files for median values of Doppler centroid (fd1), near range (near_range), and earth radius (earth_radius). An example command is:

ALOS_pre_process IMG-HH-ALPSRP022200660-H1.0__A LED-ALPSRP022200660-H1.0_AA

In this case there are 27 images so one creates a script to pre-process all 27 images.

2) Based on this analysis one preprocesses all the images using the batch processing command.

pre_proc_batch.csh ALOS data.in 
The file data.in defines the zero-baseline image and the common parameters for the stack.

IMG-HH-ALPSRP055750660-H1.0__A -near 846567 -radius 6371902.401705 -fd1 100

IMG-HH-ALPSRP022200660-H1.0_A

IMG-HH-ALPSRP028910660-H1.0_A

IMG-HH-ALPSRP035620660-H1.0_A

This command will also create a baseline versus time plot as shown in Figure 6.

3) The next step is to select the master image somewhere in the center of the baseline versus time plot. The objective in this case is to form long time span interferograms having relatively short baselines so the user connects candidate interferograms as shown in the grey lines on Figure 6. The correlation of an interferogram deteriorates as the image alignment degrades so optimally one would cross correlate each interferometric pair. However, since we would like to be able to stack interferograms and apply a uniform topographic phase correction it is advantageous to have all the images in the stack at least crudely ( $<2$ pixels) aligned. In this case we only plan to make interferograms among images that are nearby in baseline so image alignment only needs to be accurate locally. Our approach is to have a multi-step alignment process where a set of images near the master in baseline vs. time space are aligned directly to the master (primary match red dots in Figure 6). Once this set is aligned we can treat them each as surrogate masters and align images on the periphery of the baseline versus time plot to one of the nearby surrogates. We call this a secondary match (yellow dots in Figure 6). For images very far from the master we can perform a tertiary match.

4) The user puts this alignment information into a file align.in an executes the command: align_batch.csh ALOS align.in

All of the information on primary, secondary, and tertiary alignment is contained in the align.in file which is constructed by the user based on the baseline versus time plot. The example for the set shown in Figure 6 follows 
IMG-HH-ALPSRP055750660-H1.0_A:IMG-HH-ALPSRP028910660-H1.0_A:IMG-HH-ALPSRP055750660-H1.0_A IMG-HH-ALPSRP055750660-H1.0_A:IMG-HH-ALPSRP035620660-H1.0_A:IMG-HH-ALPSRP055750660-H1.0_A IMG-HH-ALPSRP055750660-H1.0_A:IMG-HH-ALPSRP042330660-H1.0_A:IMG-HH-ALPSRP055750660-H1.0_A IMG-HH-ALPSRP055750660-H1.0_A:IMG-HH-ALPSRP049040660-H1.0_A:IMG-HH-ALPSRP055750660-H1.0_A IMG-HH-ALPSRP055750660-H1.0_A:IMG-HH-ALPSRP062460660-H1.0_A:IMG-HH-ALPSRP055750660-H1.0_A IMG-HH-ALPSRP055750660-H1.0_A:IMG-HH-ALPSRP075880660-H1.0_A:IMG-HH-ALPSRP055750660-H1.0_A IMG-HH-ALPSRP055750660-H1.0_A:IMG-HH-ALPSRP082590660-H1.0_A:IMG-HH-ALPSRP055750660-H1.0_A IMG-HH-ALPSRP055750660-H1.0_A:IMG-HH-ALPSRP089300660-H1.0_A:IMG-HH-ALPSRP055750660-H1.0_A IMG-HH-ALPSRP055750660-H1.0_A:IMG-HH-ALPSRP096010660-H1.0_A:IMG-HH-ALPSRP055750660-H1.0_A IMG-HH-ALPSRP055750660-H1.0_A:IMG-HH-ALPSRP129560660-H1.0_A:IMG-HH-ALPSRP055750660-H1.0_A IMG-HH-ALPSRP055750660-H1.0_A:IMG-HH-ALPSRP163110660-H1.0_A:IMG-HH-ALPSRP055750660-H1.0_A IMG-HH-ALPSRP055750660-H1.0_A:IMG-HH-ALPSRP183240660-H1.0_A:IMG-HH-ALPSRP055750660-H1.0_A IMG-HH-ALPSRP055750660-H1.0_A:IMG-HH-ALPSRP189950660-H1.0_A:IMG-HH-ALPSRP055750660-H1.0_A IMG-HH-ALPSRP049040660-H1.0_A:IMG-HH-ALPSRP022200660-H1.0_A:IMG-HH-ALPSRP055750660-H1.0_A IMG-HH-ALPSRP049040660-H1.0_A:IMG-HH-ALPSRP142980660-H1.0_A:IMG-HH-ALPSRP055750660-H1.0_A IMG-HH-ALPSRP049040660-H1.0_A:IMG-HH-ALPSRP149690660-H1.0_A:IMG-HH-ALPSRP055750660-H1.0_A IMG-HH-ALPSRP049040660-H1.0_A:IMG-HH-ALPSRP156400660-H1.0_A:IMG-HH-ALPSRP055750660-H1.0_A IMG-HH-ALPSRP075880660-H1.0_A:IMG-HH-ALPSRP196660660-H1.0_A:IMG-HH-ALPSRP055750660-H1.0_A IMG-HH-ALPSRP082590660-H1.0_A:IMG-HH-ALPSRP210080660-H1.0_A:IMG-HH-ALPSRP055750660-H1.0_A IMG-HH-ALPSRP096010660-H1.0_A:IMG-HH-ALPSRP109430660-H1.0_A:IMG-HH-ALPSRP055750660-H1.0_A IMG-HH-ALPSRP096010660-H1.0_A:IMG-HH-ALPSRP116140660-H1.0_A:IMG-HH-ALPSRP055750660-H1.0_A IMG-HH-ALPSRP096010660-H1.0_A:IMG-HH-ALPSRP122850660-H1.0_A:IMG-HH-ALPSRP055750660-H1.0_A IMG-HH-ALPSRP096010660-H1.0_A:IMG-HH-ALPSRP223500660-H1.0_A:IMG-HH-ALPSRP055750660-H1.0_A IMG-HH-ALPSRP096010660-H1.0_A:IMG-HH-ALPSRP230210660-H1.0_A:IMG-HH-ALPSRP055750660-H1.0_A IMG-HH-ALPSRP022200660-H1.0_A:IMG-HH-ALPSRP136270660-H1.0_A:IMG-HH-ALPSRP055750660-H1.0_A

The first image is the name of the master or surrogate master, the second image is the slave to be aligned, and the third image is the supermaster that is common to all images. Note that the order of the alignment is sometimes important since the surrogate master needs to be aligned to the master before it can serve as a surrogate. Aligning a stack of 27 images can take quite a bit of computer time so it is best to do this is a batch mode and head home for the evening.

5) While waiting for the image alignment, one can construct the topographic phase using the master image. Generate a dem.grd using the web site (http://topex.ucsd.edu/gmtsar) and place it in a subdirectory /topo at the same level as /raw and /SLC.

6) Make a set of interferograms using the command intf_batch.csh ALOS intf.in intf.config

The an example file for intf.config can be copied from the location

GMTSAR/gmtsar/csh/example.intf.config and edited for this particular data set. The file intf.in contains a list of the interferometric pairs. In this case we make 22 interferograms (Figure 7).

IMG-HH-ALPSRP022200660-H1.0_A: IMG-HH-ALPSRP136270660-H1.0_A IMG-HH-ALPSRP028910660-H1.0_A: IMG-HH-ALPSRP163110660-H1.0_A IMG-HH-ALPSRP035620660-H1.0_A: IMG-HH-ALPSRP183240660-H1.0_A IMG-HH-ALPSRP035620660-H1.0_A: IMG-HH-ALPSRP189950660-H1.0_A IMG-HH-ALPSRP042330660-H1.0_A: IMG-HH-ALPSRP196660660-H1.0_A 


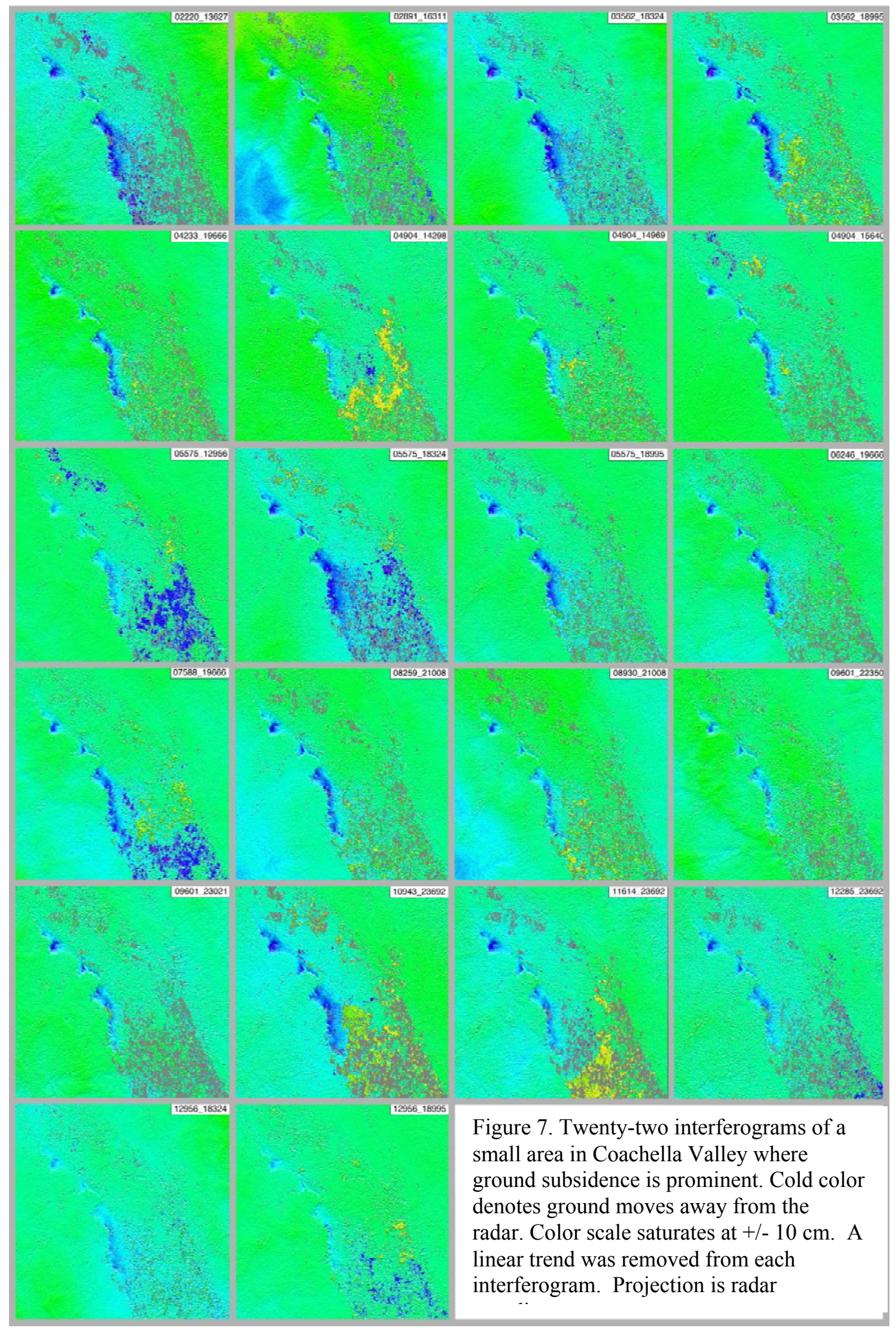




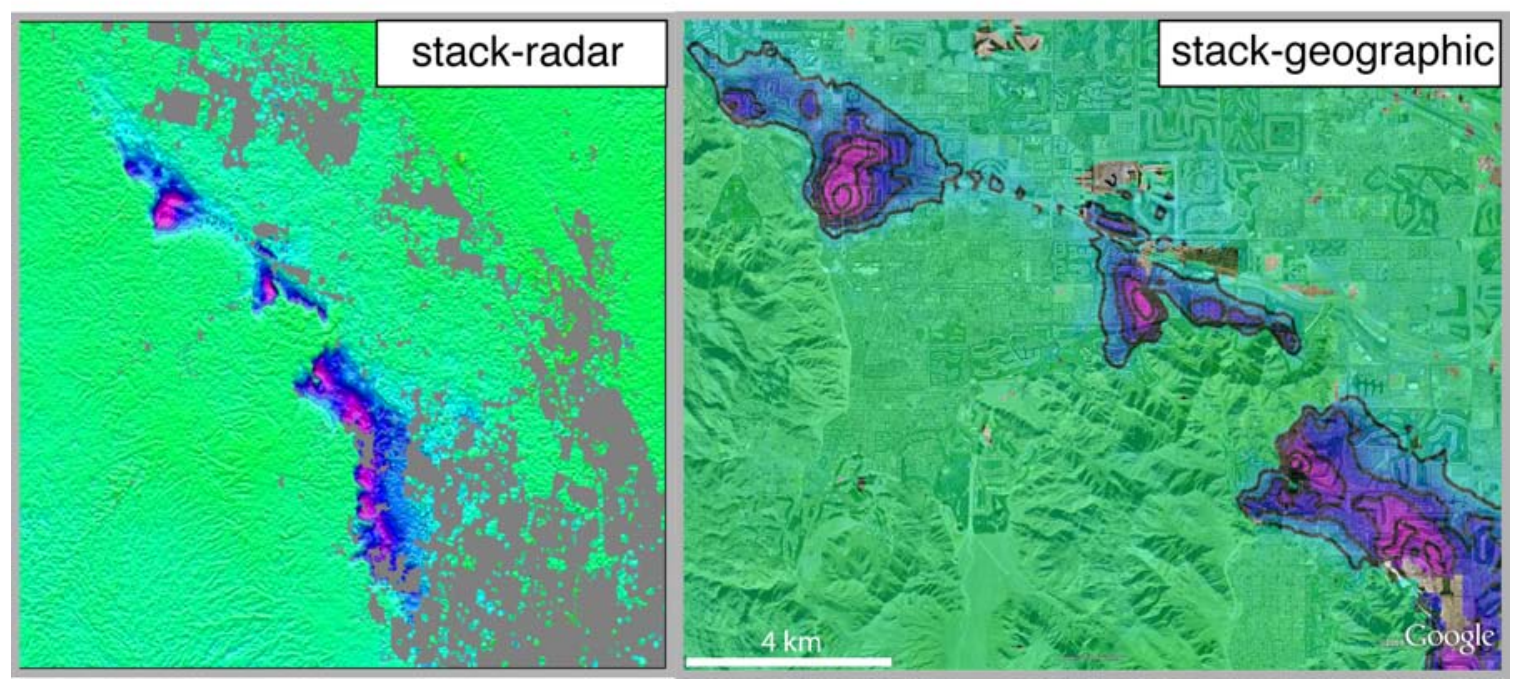

Figure 8. Stack of 22 interferograms spanning a total interval of 48 years. Contours are $1 \mathrm{~cm} / \mathrm{yr}$ LOS displacement toward radar (mostly vertical in this case). (left) radar coordinates and (right) geographic coordinates. The command grd $2 \mathrm{kml} . \mathrm{csh}$ is used to create a kml-file for Google Earth. Subsidence rates exceeding $4 \mathrm{~cm} / \mathrm{y}$ occur in Palm Desert, Indian Wells, and La Quinta areas due to groundwater pumping [USGS, 2007].

\subsection{ScanSAR Interferometry}

GMTSAR can perform two types of ScanSAR interferometry as more fully described in Appendix D. ScanSAR to ScanSAR interferometry is possible when the reference and repeat bursts overlap by more than about $20 \%$ but one must be very lucky to have more than $20 \%$ overlap. The second approach, that is always possible, is ScanSAR to strip mode interferometry. The only difference between Scan to strip processing and strip to strip processing is the preprocessing steps to convert a one of the subswaths of the ScanSAR data into a file that looks like a standard strip-mode raw data file. Here we provide a step-by-step example of processing a ScanSAR to strip (FBD) interferogram over Los Angeles Basin (Figure 9). This interferogram has a perpendicular baseline of $108 \mathrm{~m}$ and time span of 184 days. The track number is 538 and the frame number is 2930 for FBD and 2950 for ScanSAR. 


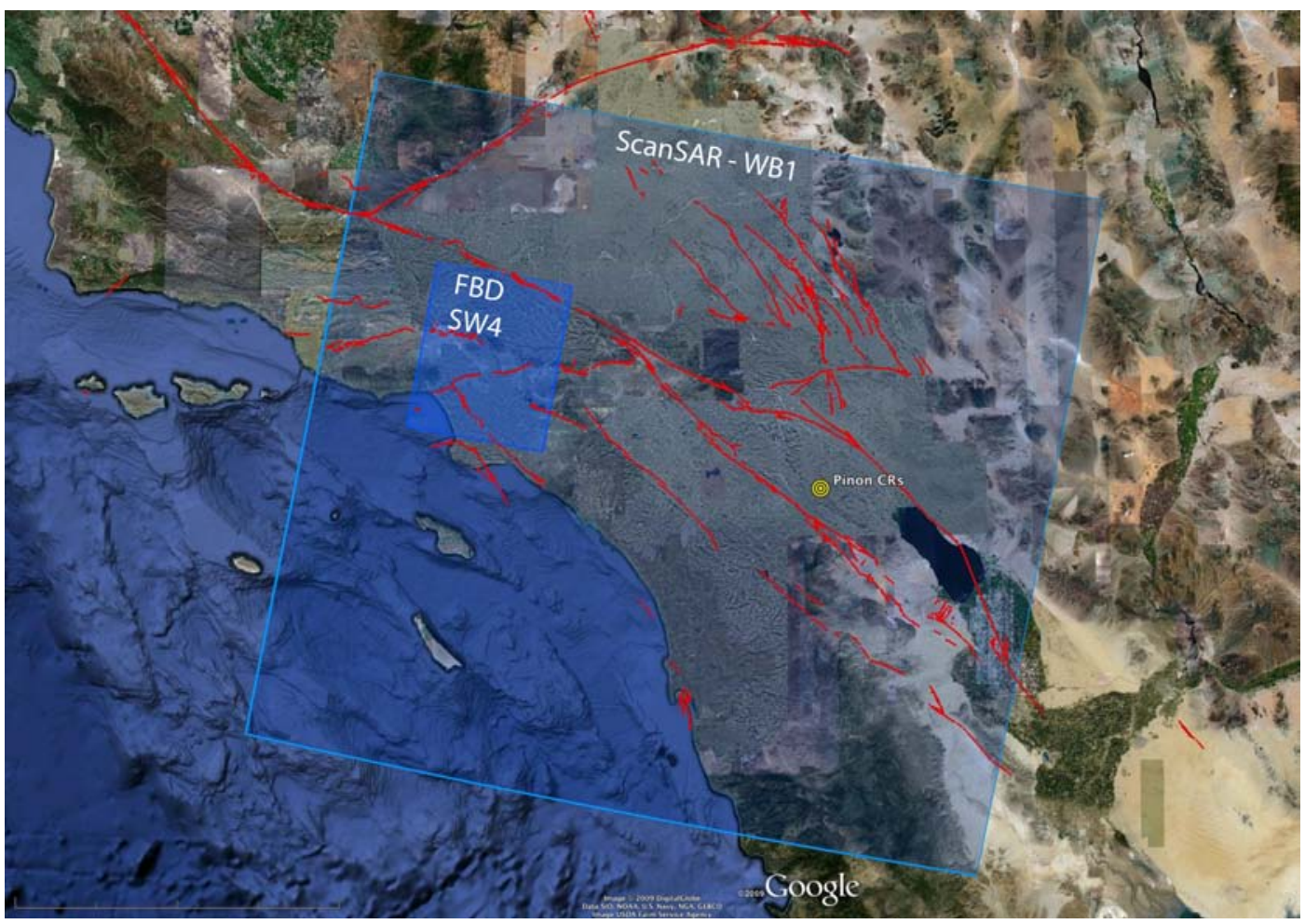

Figure 9 Location of ALOS Scansar and FBD acquisition over Los Angeles for track 538. Subswath 4 (SW4) has the same look angle as standard strip-mode data and also a similar PRF.

Table 4 Nominal radar parameters for each ScanSAR sub swath. The number of echoes in a burst nburst is the only fixed parameter.

\begin{tabular}{|c|c|c|c|c|c|}
\hline & SW1 & SW2 & SW3 & SW4 & SW5 \\
\hline near range $(\mathrm{m})$ & 730097 & 770120 & 806544 & 848515 & 878195 \\
\hline PRF $(\mathrm{Hz})$ & 1692 & 2370 & 1715 & 2160 & 1916 \\
\hline nburst & 247 & 356 & 274 & 355 & 327 \\
\hline$\Delta t(\mathrm{~s})$ & 0.146 & 0.150 & 0.160 & 0.164 & 0.171 \\
\hline nsamples & 4976 & 4720 & 5376 & 4432 & 4688 \\
\hline off nadir (deg) & 20.1 & 26.1 & 30.6 & 34.1 & 36.5 \\
\hline
\end{tabular}

The processing steps are:

1) Preprocessing FBD raw data is done by $A L O S \_$_pre_process. Unlike the above case where we convert all the lower bandwidth (FBD) data to the higher bandwidth (FBS) mode, we keep the strip data in the FBD mode because this matches the bandwidth of the ScanSAR data. Preprocessing a subswath of ScanSAR raw data is done using the program ALOS_pre_process_SS. This pre_processor has many options as shown next. 


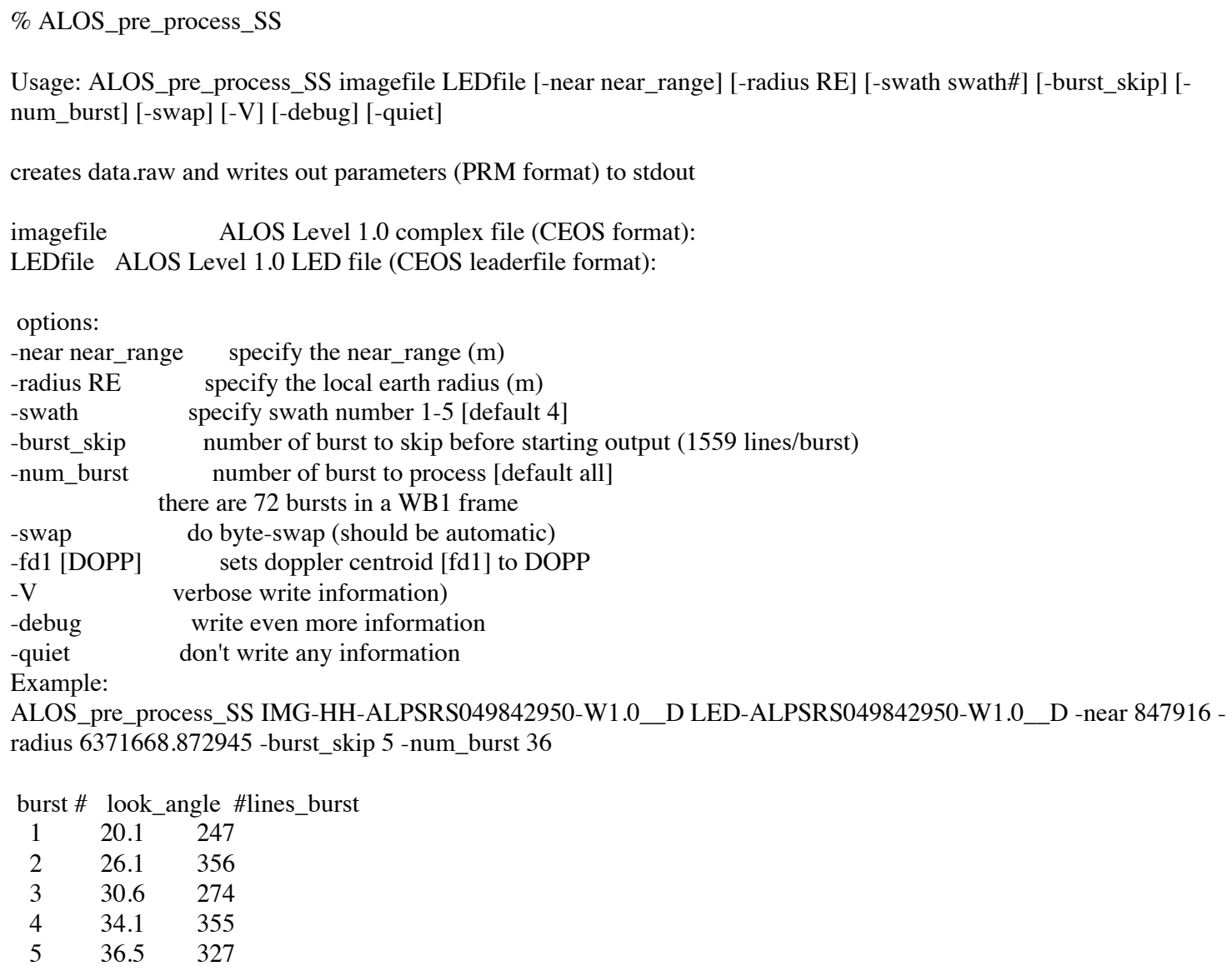

By default the $A L O S \_p r e \_p r o c e s s \_S S$ uses subswath 4, which naturally overlaps the swath mode imagery along the same track. The num_burst parameter represents the number of burst that will be extracted from a long subswath of ScanSAR. The burst_skip parameter controls the number of burst to skip before preprocessing raw data and it is determined by trial and error. After PRM files of both the ScanSAR mode and FBD mode are generated, the user runs $A L O S \_$baseline to see if the $a \_s h i f t$ value is reasonable. If not, the user chooses another burst_skip parameter and repeats this process until the a_shift is less than 1703. For this LA example the following command was used.

ALOS_pre_process_SS IMG-HH-ALPSRS049842950-W1.0_D LED-ALPSRS049842950-W1.0_D -nodopp -near 847916 -radius 6371729.321330 -num_burst 18 -burst_skip 5

Note that 18 bursts were extracted which will match the length of a single FBD frame. As described above frame-by-frame processing is usually more reliable than processing very long swaths. The $A L O S \_p r e \_p r o c e s s \_S S$ terminates when it reaches the end of the 
data file or the PRF changes in any of the subswaths. With some experimentation one can set the burst_skip to skip over the PRF change and start a fresh swath.

2) As described in Appendix D, the $A L O S \_$pre_process_SS simply inserts zero for the the missing lines in each subswath to create a standard strip-model file. To achieve optimal correlation between the scan and strip combination, one needs to zero the matching lines in the FBD data [Ortiz and Zebker, 2007]. This step is performed by the $A L O S \_f b d 2 s s$ which simply replaces real data with zeroes! The next task is to determine where to insert the zeros into the FBD data. Two parameters are needed to account for the shift and stretch between the two acquisitions. The ashift is the azimuth shift needed to align the first row of the FBD raw data to the subswath of ScanSAR data and the a_stretch_a accounts for the PRF difference. These parameters can be obtained by aligning the ScanSAR SLC with the FBD SLC images using align.csh. Essential (but not complete) steps in this alignment are listed below to guide the reader.

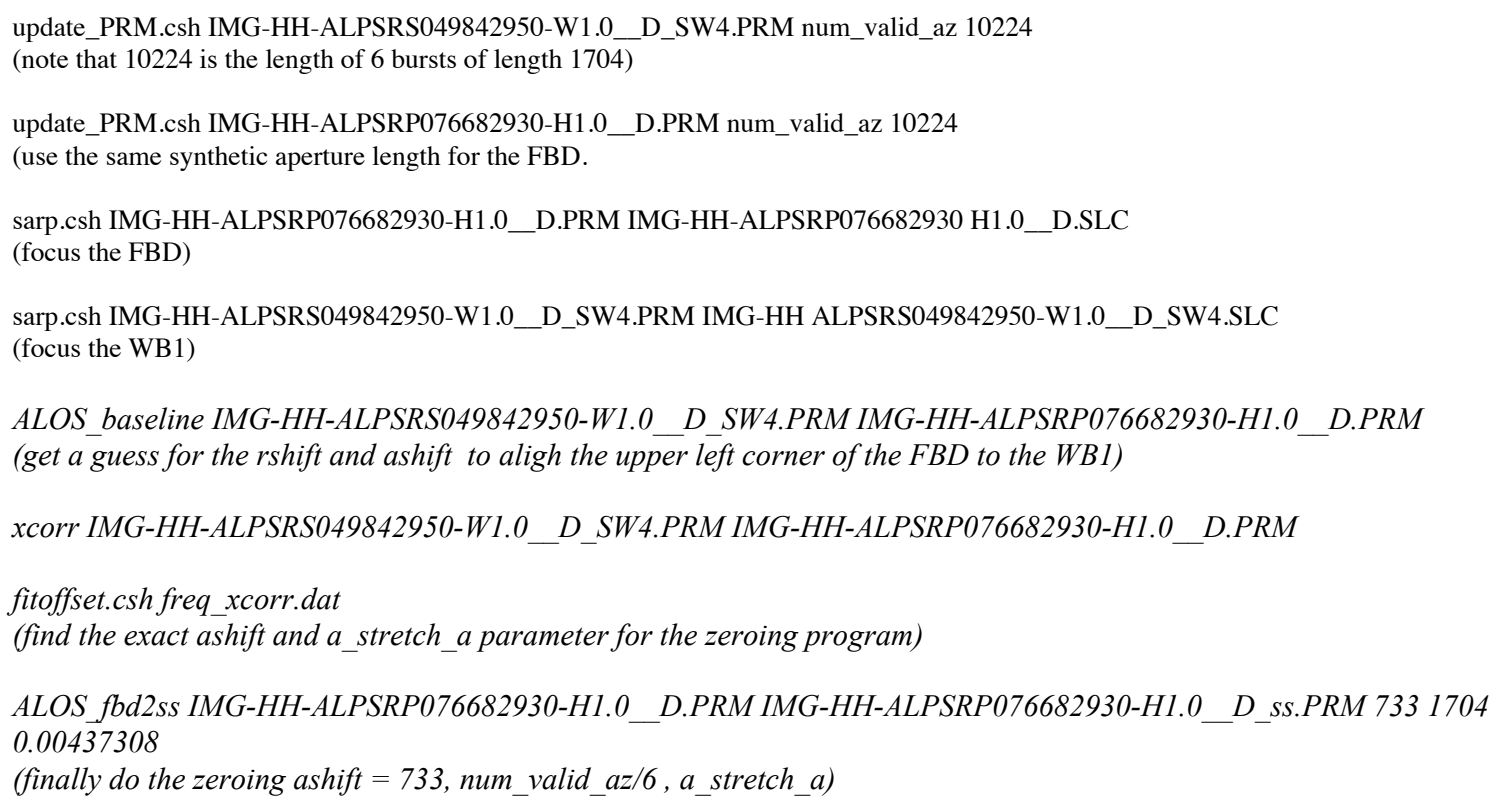

Note the rshift and ashift of the IMG-HH-ALPSRP076682930-H1.0__D_ss.PRM should be reset to zero after $A L O S \_f b d 2 s s$.

3) After the $A L O S \_f b d 2 s s$ the special preprocessing steps are completed, the data are processed as one would normally process two strip-mode acquisitions similar to the steps found in process 2 pass.csh. The essential (but not complete) steps are listed below. Note 
process 2 pass.csh can't be used directly because of the non standard filenames; some creative scripting or links could fix solve this issue.

align.csh ALOS IMG-HH-ALPSRS049842950-W1.0__D_SW4 IMG-HH-ALPSRP076682930-H1.0_D_ss

dem2topo_ra.csh master.PRM dem.grd

intf.csh IMG-HH-ALPSRS049842950-W1.0__D_SW4.PRM IMG-HH-ALPSRP076682930-H1.0_D_ss.PRM -topo topo_ra.grd

filter.csh IMG-HH-ALPSRS049842950-W1.0__D_SW4.PRM IMG-HH-ALPSRP076682930-H1.0_D_ss.PRM gauss_alos_300m 2

geocode.csh 0.1

The processed interferogram is shown in Figure 10. To make the interferogram more interesting we did not apply the topographic phase correction. During this analysis we also processed a standard FBD to FBD interferogram. For these two interferograms of the same area and about the same time interval, the average coherence of the ScanSAR to FBD interferogram was lower (0.38) than the FBD to FBD interferogram (0.67). Therefore there are really no advantages to ScanSAR to strip-mode interferometry except that it provides more interferometric opportunities in case of events.

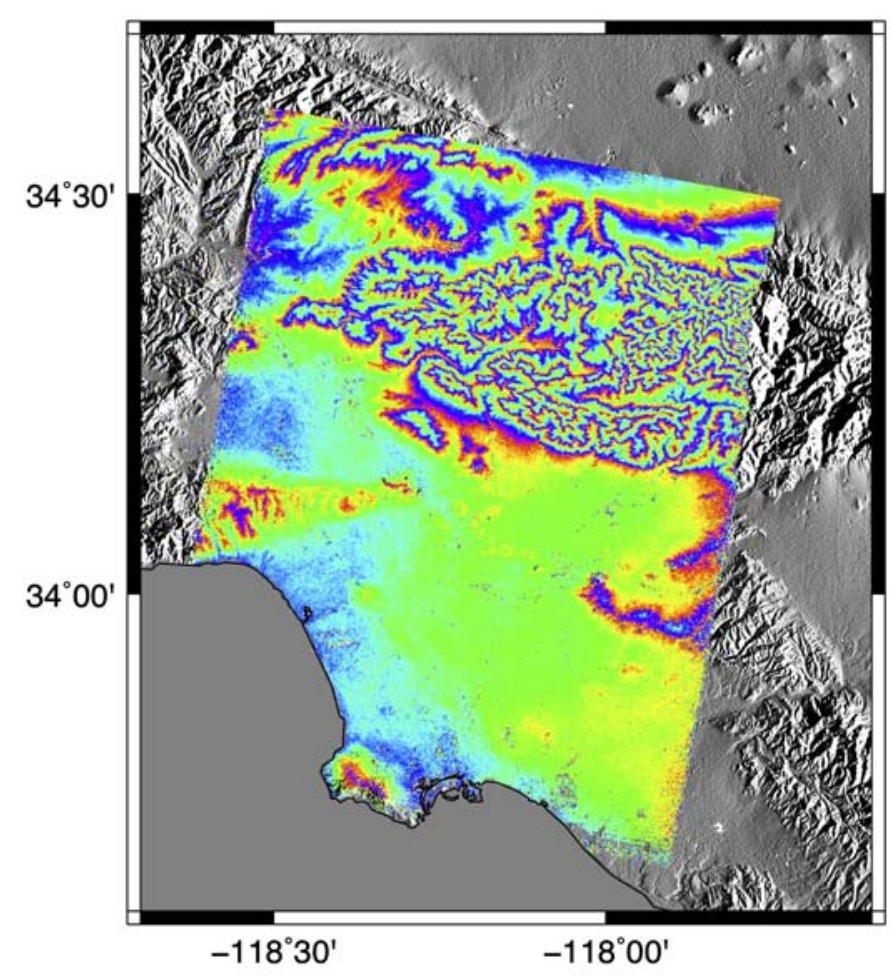

Figure 10. An example of ScanSAR to FBD mode interferogram over Los Angeles Basin where the topographic phase has not been removed.

ScanSAR to ScanSAR processing is very similar to the sequence described above although one must be lucky to find a pair having sufficient burst overlap. For each WB1 
data file one creates 5 independent interferograms for each of the 5 subswaths. As shown in Table 4, each subswath has a very different PRF so the interferograms are not easily mosaicked in the radar coordinates. However, if the data are processed with a consistent earth radius, the interferograms will abut seamlessly when they are projected back into geographic coordinates as shown in Figure 11.

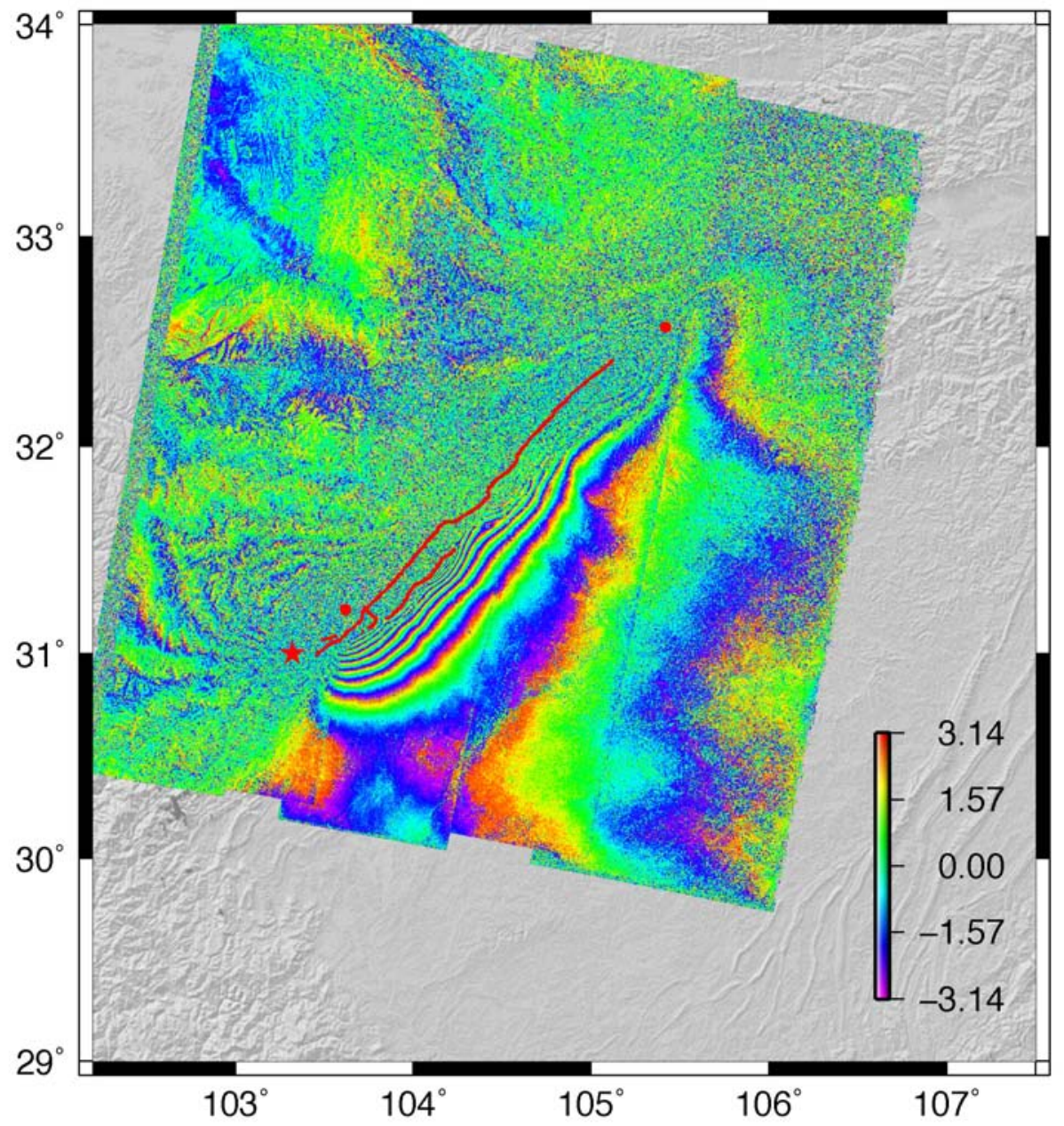

Figure 11. Descending ScanSAR to ScanSAR interferogram spanning the Mw 7.9 2008 Wenchuan earthquake (11.8 cm per fringe). The interferogram consists of 5 sub-swaths across look direction that abut almost seamlessly with no position or phase adjustment. The decorrelation in the mountainous area to the left of the red surface rupture line is due to the rather long baseline $(920 \mathrm{~m})$ and high relief. 


\section{References}

Bertran-Ortiz, A., and H.A. Zebker (2007), ScanSAR-to-Stripmap Mode Interferometry Processing Using ENVISAT/ASAR Data, IEEE Trans. Geosci. Rem. Sensing, Vol. 45, No. 11 , pp. 3468-3480.

Chen C. W. and H. A. Zebker, Network approaches to two-dimensional phase unwrapping: intractability and two new algorithms, Journal of the Optical Society of America A, vol. 17, pp. 401-414 (2000).

Curlander and McDonough, Synthetic Aperture Radar: Systems \& Signal Processing, Chapter 4, John Wiley \& Sons, New York, 1991.

Farr, T. G., et al. (2007), The Shuttle Radar Topography Mission, Rev. Geophys., 45, RG2004, doi:10.1029/2005RG000183.

Press, W.H., Teukolsky, S.A., Vetterling, W.T. \& Flannery, B.P., 1992. Numerical recipes in C, Second Edition. Cambridge University Press, New York, 994 pp.

Sandwell, D. T. and E. J. Price, Phase gradient approach to stacking interferograms, J. Geophys. Res., 103, 30183-30204, 1998.

Sneed, M. and J. Brandt, Detection and Measurement of Land Subsidence Using GPS Surveying and InSAR, Coachella Valley, California, 1996-2006, USGS Scientific Investigations Report, 2007-5251, 41 pp., 2007

Swarztrauber, P. N., Vectorizing the FFTs, in Parallel Computations (G. Rodrigue, ed.), Academic Press, 1982, pp. 51--83.

Wessel, P., and W. H. F. Smith, New, improved version of Generic Mapping Tools released, EOS Trans. Amer. Geophys. U., vol. 79 (47), pp. 579, 1998.

\section{Problems}

1) Precise orbital information is used in 4 areas of InSAR processing. Describe the 4 uses.

2) The natural coordinates of a radar image have range or time along one axis and azimuth or slow time along the other axis. Why is the term slow time used?

3) Describe an algorithm to transform a point on the surface of the earth (longitude, latitude, elevation) into radar coordinates.

4) When a SAR image is focussed at zero Doppler, the radar coordinates of a point reflector correspond to the minimum range. Why? Derive the equations (4) and (5) for adjusting the range and azimuth coordinates when an image is focussed at a nonzero Doppler.

5) GMTSAR uses a quadratic function to approximate the changes in baseline (horizontal and vertical) along the image frame. The quadratic formula is:

$$
B(s)=a+b s+c s^{2}
$$


where $s$ is slow time ranging from the start to the end of the frame $[0, T]$. Suppose the actual baseline is measured at three times along the frame $0, \frac{T}{2}, T$ and the values are $B_{1}, B_{2}, B_{3}$. Derive an expression for the parameters $a, b, c$. The forward model is $\left(\begin{array}{l}B_{1} \\ B_{2} \\ B_{3}\end{array}\right)=\left(\begin{array}{ccc}1 & 0 & 0 \\ 1 & \frac{T}{2} & \frac{T^{2}}{4} \\ 1 & T & T^{2}\end{array}\right)\left(\begin{array}{l}a \\ b \\ c\end{array}\right)$.

6) Satellite orbital information is commonly provided as state vectors of position and velocity at regular intervals (e.g., 1 minute). Hermite polynomial interpolation is often used to interpolate the orbit to the full sampling rate of the radar (e.g., $2000 \mathrm{~Hz}$ ). What are the strengths and weaknesses of this approach? (This may require a literature search.)

7) Explain the terminology, master and slaves, reference and repeat. 
Appendix A - Principles of Synthetic Aperture Radar

\section{Fraunhoffer diffraction}

To understand why a synthetic aperture is needed for microwave remote sensing from orbital altitude one must understand the concepts of diffraction and resolution. Consider the projection pattern of coherent radiation after it passes through an aperture (Figure A1). First we'll consider a 1-D aperture and then go on to a 2-D rectangular aperture to simulate a rectangular SAR antenna. The 2-D case provides the shape and dimension of the footprint of the radar. Although we will develop the resolution characteristics of apertures as transmitters of radiation, the resolution characteristics are exactly the same when the aperture is used to receive radiation. These notes were developed from Rees [2001] and Bracewell [1978].

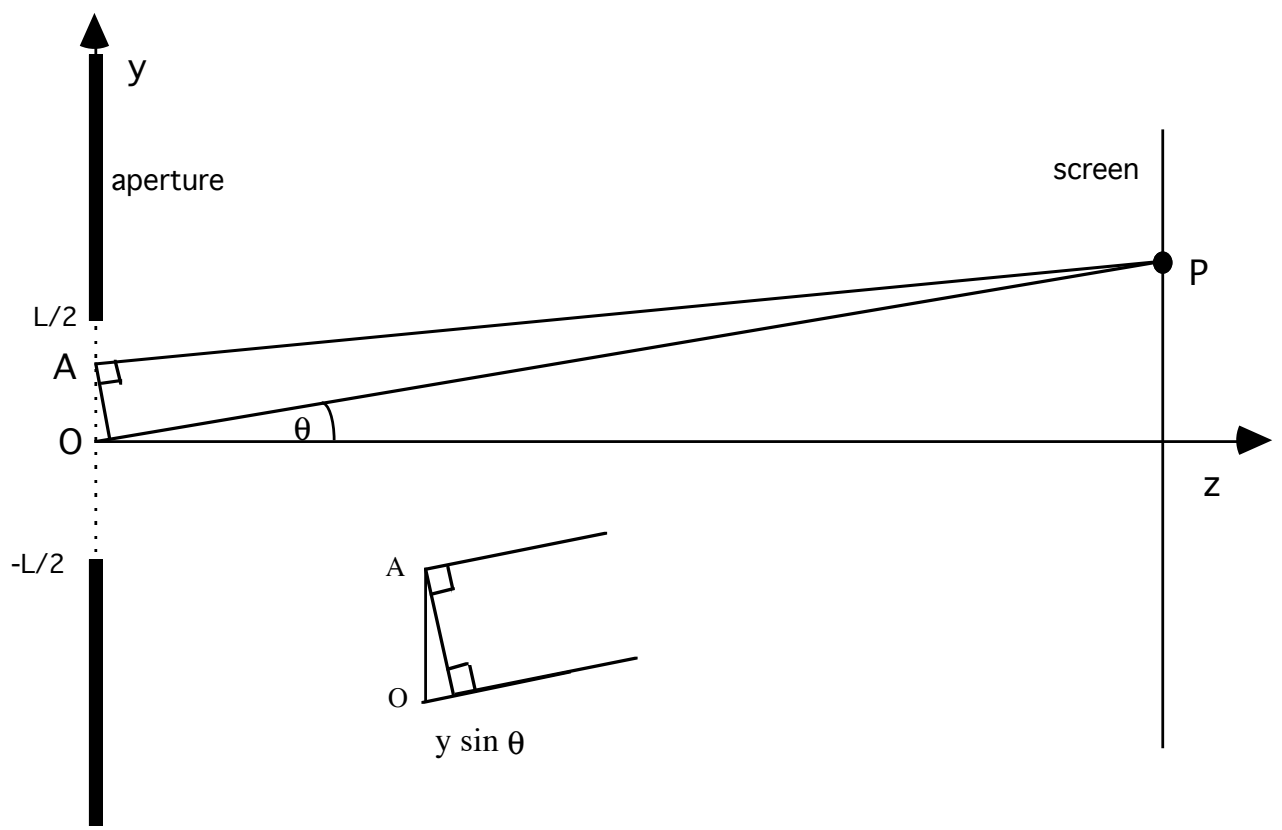

Figure A1 Diagram for the projection of coherent microwaves on a screen that is far from the aperture of length $L$.

We simulate coherent radiation by point sources of radiation distributed along the aperture between $-L / 2$ and $L / 2$. For simplicity we'll assume all the sources have the same amplitude, wavelength $\lambda$, and phase. Given these sources of radiation, we solve for the illumination pattern on the screen as a function of $\theta$. We'll assume that the screen is far enough from the aperture so that rays AP and OP are parallel. Later we'll determine how far away the screen needs to be in order for this approximation to hold. Under these 
conditions, the ray AP is slightly shorter than the ray OP by an amount $-y \sin \theta$. This corresponds to a phase shift of $\frac{-2 \pi}{\lambda} y \sin \theta$. The amplitude of the illumination at point $P$ is the integral over all of the sources along the aperture multiplied by their complex phase value

$$
P(\theta)=\int_{-L / 2}^{L / 2} A(y) e^{-i 2 \pi y k \sin \theta} d y
$$

where $k=1 / \lambda$. This is called the Fraunhoffer diffraction integral. The illumination across the aperture is uniform in both amplitude and phase so we set $A(y)=1$. Now let $s=2 \pi k \sin \theta$ so the fourier integral is easy to evaluate.

$P(s)=\int_{-L / 2}^{L / 2} e^{-i s y} d y=\frac{e^{-i s L / 2}-e^{i s L / 2}}{-i s}=\frac{2}{s} \sin (s L / 2)=L \sin c(s L / 2)$

Replacing $s$ with $2 \pi \sin \theta / \lambda$ we arrive at the final result.

$P(\theta)=L \operatorname{sinc}\left(\frac{L \pi \sin \theta}{\lambda}\right)$

The illumination pattern on the screen is shown in Figure A2.

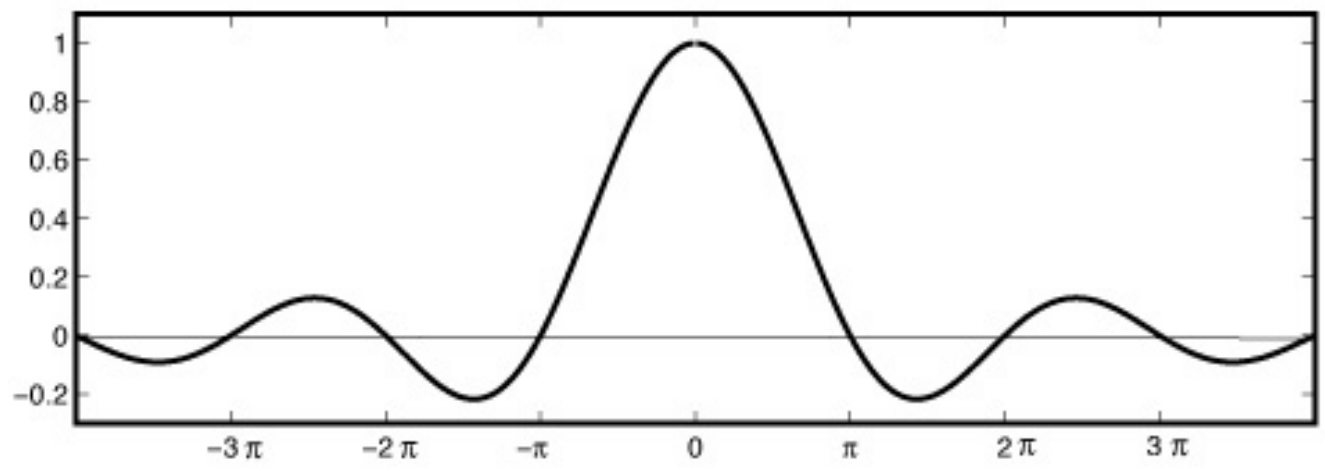

Figure A2. Sinc function illumination pattern for the aperture shown in Figure A1.

The first zero crossing, or angular resolution $\theta_{r}$ of the sinc function occurs when the argument is $\pi$ so $\sin \theta_{r}=\frac{\lambda}{L}$ and for small angles $\theta_{r} \cong \lambda / L$ and $\tan \theta_{r} \cong \sin \theta_{r}$. Note that one could modify the screen illumination by changing the strength of the illumination across the aperture. For example, a Gaussian aperture would produce a Gaussian 
illumination function on the screen. This would eliminate the sidelobes associated with the sinc function but it would also broaden the projection pattern. In addition, one could vary the phase along the aperture to shift the point of maximum illumination away from $\theta=0$. Such a phased array aperture is used in some radar systems to continuouslyilluminate a feature as the satellite passed over it. This is called spotlight mode SAR and it is a favorite technique for military reconnaissance.

One can perform the same type of analysis with any 2-D aperture; many analytic examples are given in Figure 12.4 from Bracewell [1978]. Below we'll be using a rectangular aperture when we discuss synthetic aperture radars. For now, consider a uniform circular aperture has an angular resolution given by $\sin \theta_{r}=1.22 \lambda / L$.

The Geosat radar altimeter orbits the Earth at an altitude of $800 \mathrm{~km}$ and illuminates the ocean surface with a $1-\mathrm{m}$ parabolic dish antenna operating at $\mathrm{Ku}$ band $(13.5 \mathrm{GHz}, \lambda=$ $0.022 \mathrm{~m}$ ) (Figure A3). The diameter of the beam-limited footprint on the ocean surface is $D_{s}=2 H \tan \theta_{r} \cong 2.44 H \frac{\lambda}{D}$ or in this case $43 \mathrm{~km}$.

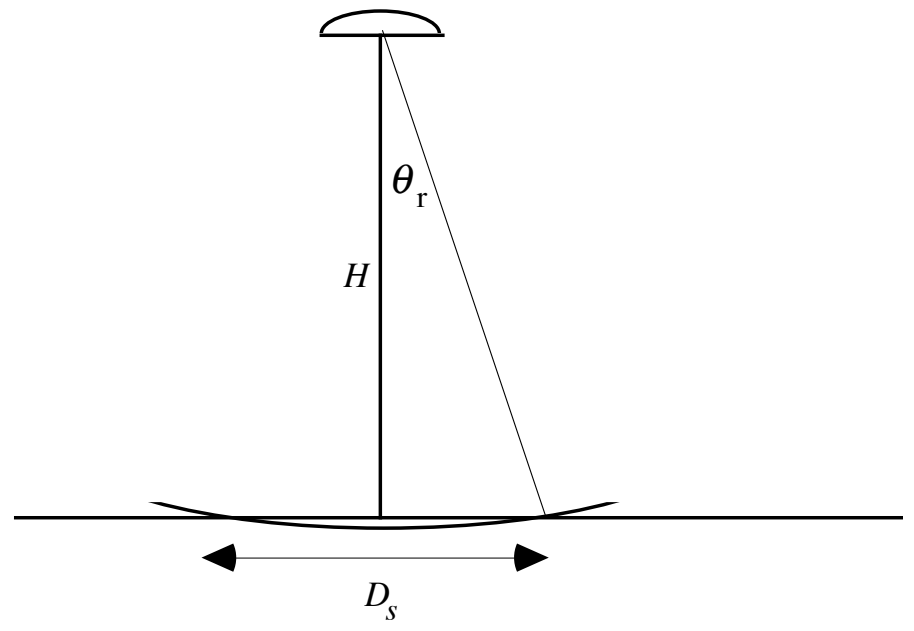

Figure A3. Beam-limited footprint $D_{s}$ of a circular aperture (radar altimeter or optical telescope) at an altitude of $H$.

An optical system with the same $1 \mathrm{~m}$ diameter aperture, but operating at a wavelength of $\lambda=5 \times 10^{-7} \mathrm{~m}$, has a footprint diameter of $0.97 \mathrm{~m}$. The Hubble space telescope reports an angular resolution of 0.1 arcsecond, which corresponds to an effective aperture of 1.27 $\mathrm{m}$. Thus a 1-m ground resolution is possible for optical systems while the same size aperture operating in the microwave part of the spectrum has a 44,000 times worse resolution. Achieving high angular resolution for a microwave system will require a major increase in the length of the aperture. 
Before moving on to the 2-D case, we should check the assumption used in developing the Fraunhoffer diffraction integral that the rays AP and OP are parallel. Suppose we examine the case when $\theta=0$; the ray path AP is slightly longer than OP (Figure A4). This parallel-ray assumption breaks down when the phase of ray path AP is more than $\pi / 2$ radians longer than OP which corresponds to a distance of $\lambda / 4$. Lets determine the conditions when this happens.

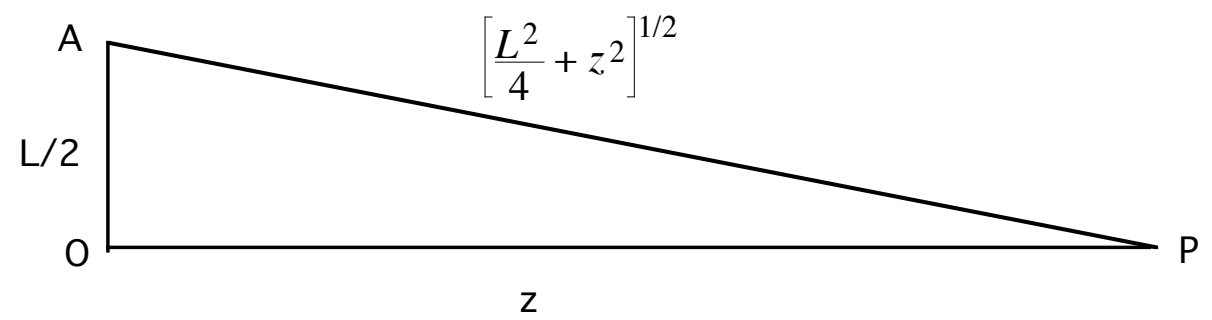

Figure A4. Diagram showing the increases length of path AP with respect to OP due to an offset of $L / 2$.

The condition that the path length difference is smaller than $1 / 4$ wavelength is

$\left[\frac{L^{2}}{4}+z^{2}\right]^{1 / 2}-z<\frac{\lambda}{4}$

or can be rewritten as

$\left[\left(\frac{L}{2 z}\right)^{2}+1\right]^{1 / 2}-1<\frac{\lambda}{4 z}$

Now assume $L<<z$ so we can expand the term in bracket in a binomial series.

$1+\frac{L^{2}}{8 z^{2}}-1<\frac{\lambda}{4 z}$

and we find $z_{f}>\frac{L^{2}}{2 \lambda}$ where $z_{f}$ is the Fresnel distance. So when $z<z_{f}$ we are in the near field and we need to use a more rigorous diffraction theory. However when $z>>z_{f}$ we 
are safe to use the parallel-ray approximation and the Fraunhoffer diffraction integral is appropriate. Next consider some examples.

Geosat; $\quad L=1 \mathrm{~m}, \lambda=0.022 \mathrm{~m} \quad z_{f}=23 \mathrm{~m}$

At an orbital altitude of $800 \mathrm{~km}$ the parallel-ray approximation is valid.

Optical telescope; $\quad L=1 \mathrm{~m}, \lambda=5 \times 10^{-7} \mathrm{~m} \quad z_{f}=4000 \mathrm{~km}$.

So we see that an optical system with an orbital altitude of $800 \mathrm{~km}$ will require near-field optics.

What about a synthetic aperture radar (discussed below) such as ERS with a $4000 \mathrm{~m}$ long synthetic aperture.

ERS SAR; $\quad L=4000 \mathrm{~m}, \lambda=0.058 \mathrm{~m} \quad z_{f}=140,000 \mathrm{~km}$.

Thus near-field optics are also required to achieve full SAR resolution for ERS. This near-field correction is done in the SAR processor by performing a step called range migration and it is a large factor in making SAR processing so CPU-intensive.

\section{2-D Aperture - ERS example}

A 2-D rectangular aperture is a good approximation to a typical spaceborne strip-mode SAR. The aperture is longer in the flight direction (length $L$ ) than in the flight perpendicular direction (width $W$ ) as shown in Figure A5. As in the 1-D case, one uses a 2-D Fraunhoffer diffraction integral to calculate the projection pattern of the antenna. The integral is

$$
P\left(\theta_{x}, \theta_{y}\right)=\int_{-L / 2}^{L / 2} \int_{-W / 2}^{W / 2} A(x, y) \exp \left[i \frac{2 \pi}{\lambda}\left(x \sin \theta_{x}+y \sin \theta_{y}\right)\right] d x d y
$$

where $\lambda$ is the wavelength of the radar. As in the 1-D case we'll assume the aperture $A(x, y)$ has uniform amplitude and phase. In this case the projection pattern can be integrated analytically and is

$$
P\left(\theta_{x}, \theta_{y}\right)=L W \operatorname{sinc}\left(\frac{\pi W \sin \theta_{x}}{\lambda}\right) \operatorname{sinc}\left(\frac{\pi L \sin \theta_{y}}{\lambda}\right) .
$$

The first zero crossing of this 2-D sinc function is illustrated in Figure A5 (right). 

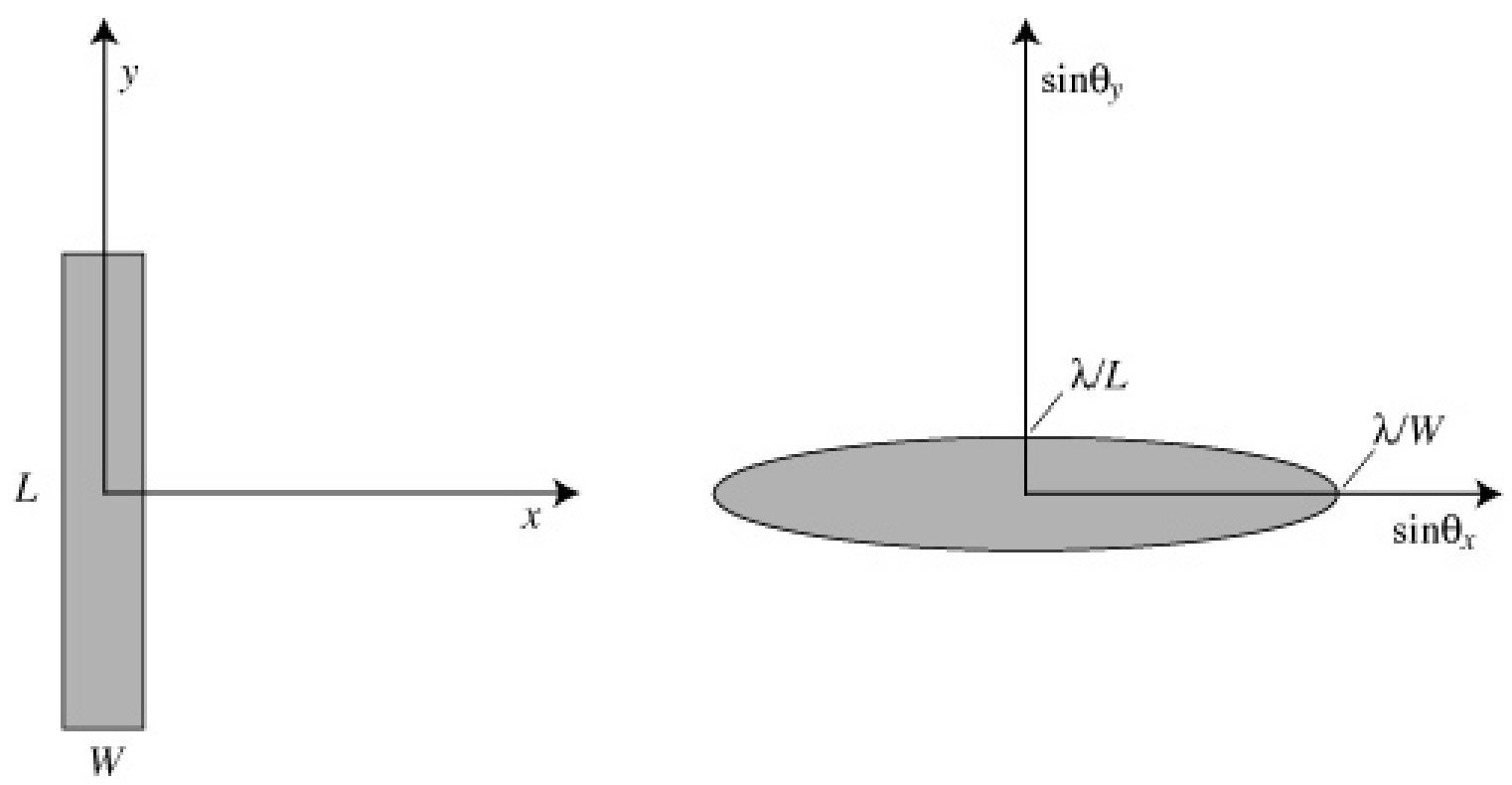

Figure A5 Diagram showing the projection pattern (right) for a rectangular SAR antenna (left).

The ERS radar has a wavelength of $0.05 \mathrm{~m}$, an antenna length $L$ of $10 \mathrm{~m}$, and an antenna width $W$ of $1 \mathrm{~m}$. For a nominal look angle of $23^{\circ}$ the slant range $R$ is $850 \mathrm{~km}$. The footprint of the radar has an along-track dimension $D_{a}=2 R \lambda / L$, which is $8.5 \mathrm{~km}$. As discussed below this is approximately the length of the synthetic aperture used in the SAR processor. The footprint in the range direction is 10 times larger or about $85 \mathrm{~km}$.

\section{Range resolution (end view)}

The radar emits a short pulse that reflects off the surface of the earth and returns to the antenna. The amplitude versus time of the return pulse is a recording of the reflectivity of the surface. If adjacent reflectors appear as two distinct peaks in the return waveform then they are resolved in range. The nominal slant range resolution is $\Delta r=C \tau / 2$ where $\tau$ is the pulse length, and $C$ is the speed of light. The factor of 2 accounts for the 2-way travel time of the pulse. Figure A6 shows how the ground range resolution is geometrically related to the slant range resolution $R_{r}=\frac{C \tau}{2 \sin \theta}$. 


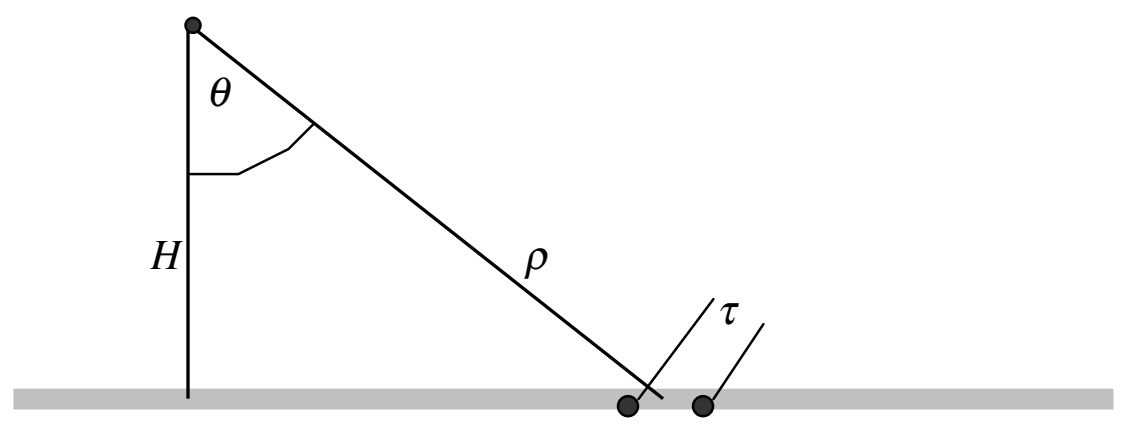

$\begin{array}{lll}\theta & - & \text { look angle } \\ H & - & \text { spacecraft height } \\ B & - & \text { bandwidth of radar } \\ \tau & - & \text { pulse length } 1 / B \\ C & - & \text { speed of light }\end{array}$

Figure A6. Diagram of radar flying into the page emitting a pulse of length $\tau$. That reflects from two points on the surface of the earth.

Note the ground range resolution is infinite for vertical look angle and improves as look angle is increased. Also note that the range resolution is independent of the height of the spacecraft $H$. The range resolution can be improved by increasing the bandwidth of the radar. Usually the radar bandwidth is a small fraction of the carrier frequency so shorter wavelength radar does not necessarily enable higher range resolution. In many cases the bandwidth of the radar is limited by the speed at which the data can be transmitted from the satellite to a ground station.

\section{Azimuth resolution (top view)}

To understand the azimuth resolution, consider a single point reflector on the ground that is illuminated as the radar passes overhead (Figure A7). From the Fraunhoffer diffraction analysis we know the length of the illumination (twice the angular resolution) is related to the wavelength of the radar divided by the length of the antenna. As discussed above the along-track dimension of the ERS footprint is $8.5 \mathrm{~km}$ so the nominal resolution $R_{a}$ is $4.25 \mathrm{~km}$, which is very poor. This is the azimuth resolution of the realaperture radar 


$$
R_{a}=\rho \tan \theta_{r} \cong \frac{\rho \lambda}{L}=\frac{\lambda H}{L \cos \theta}
$$

where

$\begin{array}{lll}L & - & \text { length of radar antenna } \\ \rho & - & \text { nominal slant range } H / \cos \theta \\ \lambda & - & \text { wavelength of radar. }\end{array}$

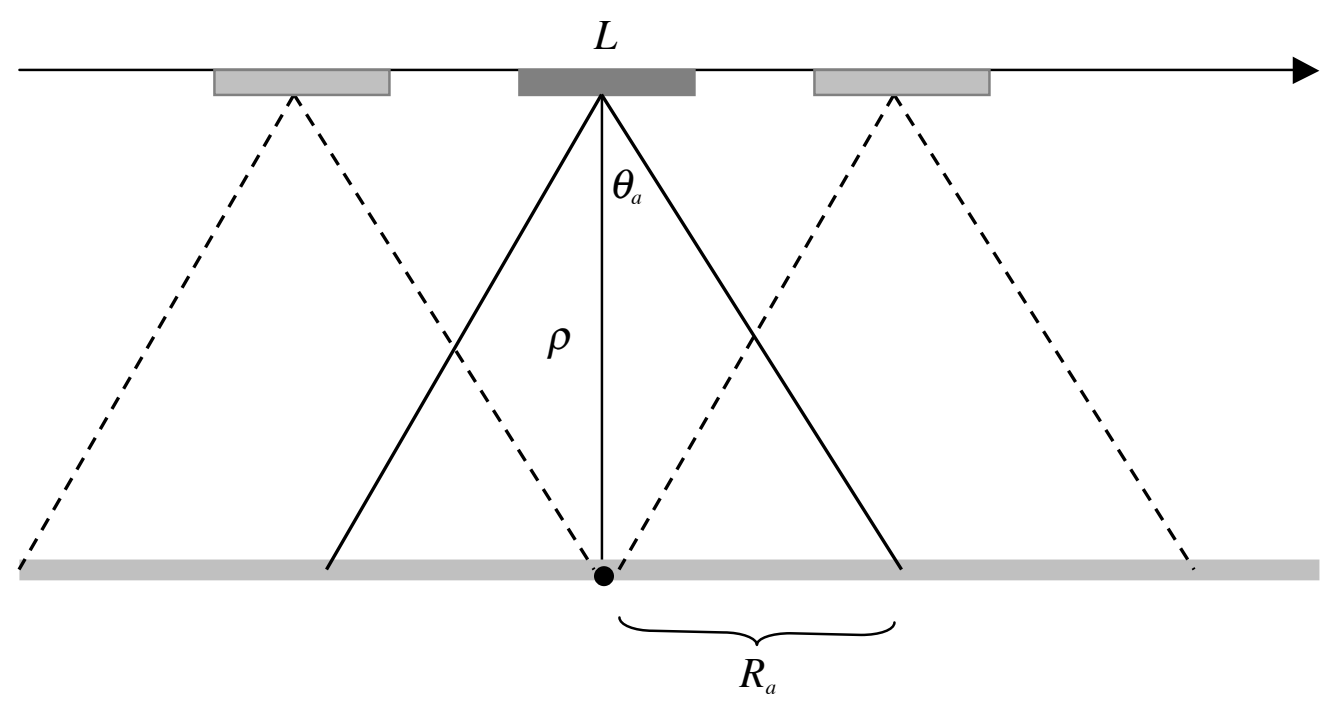

Figure A7. Top view of SAR antenna imaging a point reflector. The reflector remains within the illumination pattern over the real aperture length of $2 R_{a}$.

If the scatterer on the ground remains stationary as the satellite passes overhead, then one can assemble a synthetic aperture with a length equal to the along-track beamwidth of length which is $2 R_{a}$. This much longer aperture of $8.5 \mathrm{~km}$ results in a dramatic improvement in azimuth resolution given by

$R_{a}^{\prime}=\frac{\lambda \rho}{2 R_{a}}=\frac{L}{2}$

which is the theoretical resolution of a strip-mode SAR. Note the synthetic aperture azimuth resolution is independent of spacecraft height and improves as the antenna length is reduced. One could form a longer synthetic aperture by steering the transmitted radar beam so it follows the target as the spacecraft (aircraft) flies by. This is called spotlight-mode SAR. 


\section{Range and Azimuth Resolution of ERS SAR}

The ERS SAR has a bandwidth of $15.6 \mathrm{MHz}$, an antenna length of $10 \mathrm{~m}$ and a look angle of $23^{\circ}$. Accordingly the ground range resolution is about $25 \mathrm{~m}$ and the maximum azimuth resolution is $5 \mathrm{~m}$. In practice, one averages several "looks" together to improve the quality of the amplitude (backscatter) image. In the case of ERS, one could average 5 looks for a resolution cell of $25 \mathrm{~m}$ by $25 \mathrm{~m}$.

\section{Pulse repetition frequency}

The discussion above suggests that we should make the antenna length $L$ as short as possible to improve azimuth resolution. However, to form a complete aperture without aliasing longer wavelengths back into shorter wavelengths we must pulse the radar at an along-track distance of $\mathrm{L} / 2$ or shorter. To understand why such a rapid pulse rate is necessary, consider the maximum Doppler shift from a point that is illuminated at a maximum distance ahead of the radar (Figure A8) where $v_{o}$ is the carrier frequency $=C / \lambda$ and $V$ is the velocity of spacecraft relative to the ground.

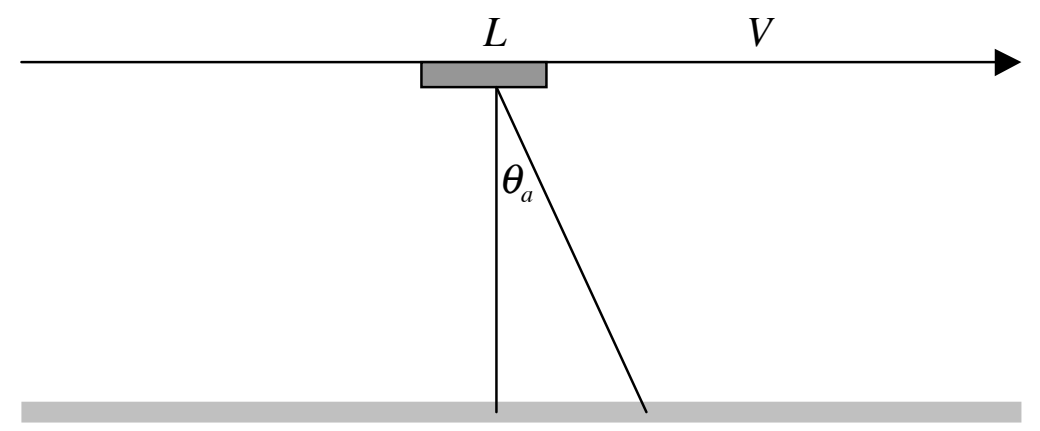

Figure A8. Diagram showing the angle between broadside to the antenna and the point where a reflector first enters the illumination of the antenna.

The maximum Doppler shift occurs at a maximum angle of

$$
\sin \theta_{a}=\frac{\lambda}{L}
$$

$$
\Delta v=2 v_{o} \frac{V}{C} \sin \theta_{a}=\frac{2 C}{\lambda} \frac{V}{C} \frac{\lambda}{L}=\frac{2 V}{L}
$$


This corresponds to a maximum along-track distance between samples of $L / 2$. For ERS this corresponds to a minimum pulse repetition frequency (PRF) of $1400 \mathrm{~Hz}$. The actual PRF of ERS is $1680 \mathrm{~Hz}$.

\section{Other constraints on the PRF}

The PRF cannot be too large or the return pulses from the near range and far range will overlap in time as shown in Figure A9.

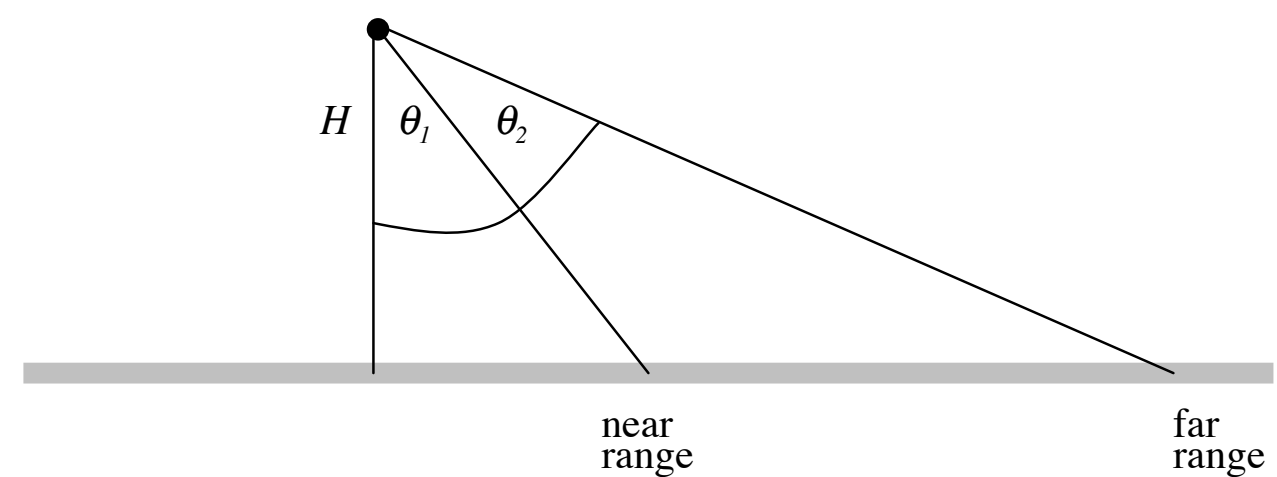

Figure A9. End view of the distance to the near range and far range of the radar illumination pattern.

$$
\begin{aligned}
& P R F<\frac{1}{t_{2}-t_{1}} \\
& t_{1}=\frac{2 H}{C \cos \theta_{1}} \quad t_{2}=\frac{2 H}{C \cos \theta_{2}} \\
& \Delta t=\frac{2 H}{C}\left(\sec \theta_{2}-\sec \theta_{1}\right) \\
& \frac{2 V}{L}<P R F<\frac{C}{2 H}\left(\sec \theta_{2}-\sec \theta_{1}\right)^{-1}
\end{aligned}
$$

For ERS the look angles to the rear range and far range are $18^{\circ}$ and $24^{\circ}$, respectively. Thus the maximum PRF is $4777 \mathrm{~Hz}$. The actual PRF of 1680 is safely below this value and the real limitation is imposed by the speed of the data link from the spacecraft to the ground. A wider swath or a higher PRF would require a faster data link than is possible using a normal X-band communication link of 105 Mbps. 


\section{References}

Bracewell, R. N. (1978), The Fourier Transform and Its Applications, second ed., 444 pp., McGraw-Hill Book Co., New York.

Curlander and McDonough, Synthetic Aperture Radar: Systems \& Signal Processing, Chapter 4, John Wiley \& Sons, New York, 1991.)

Rees, W. G. (2001), Physical Principles of Remote Sensing, second edition ed., 343 pp., Cambridge University Press, Cambridge, UK. 


\section{Problems}

1) What is the illumination pattern for an aperture with a sign reversal at its center? What is $P(0)$ ? Is the function real or imaginary? Is the function symmetric or asymmetric?

The aperture is

$$
A(y)=\left\{\begin{array}{cc}
0 & |y|>\frac{L}{2} \\
1 & 0<y \leq \frac{L}{2} \\
-1 & -\frac{L}{2} \leq y<0
\end{array}\right.
$$

2) a) What is the illumination pattern for the aperture below?

b) What is the illumination pattern in the limit as $L_{1}$ approaches $L_{2}$ ?

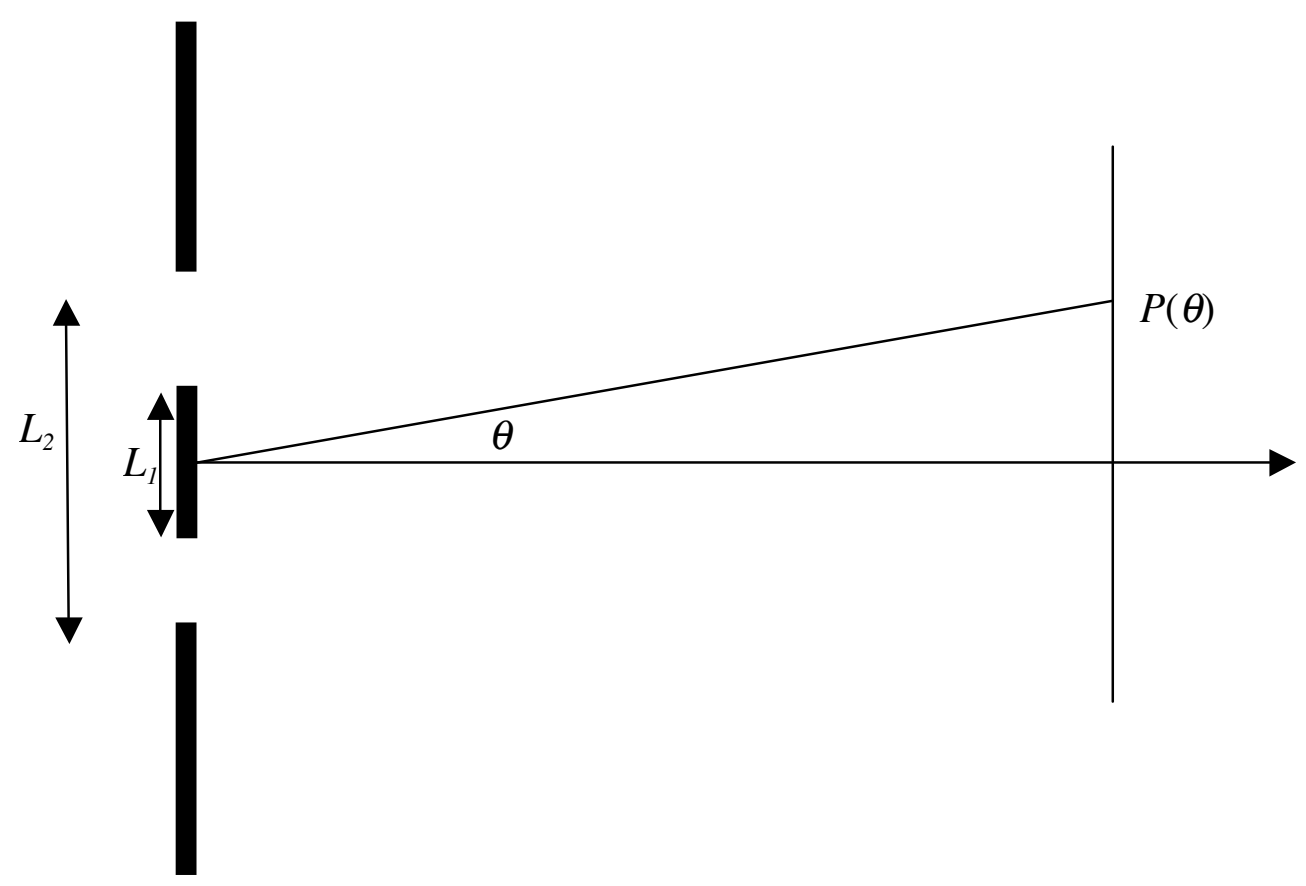


3) What is the theoretical azimuth resolution of a spotlight-mode SAR that can illuminate the target over a $10^{\circ}$ angle as shown in the diagram below.

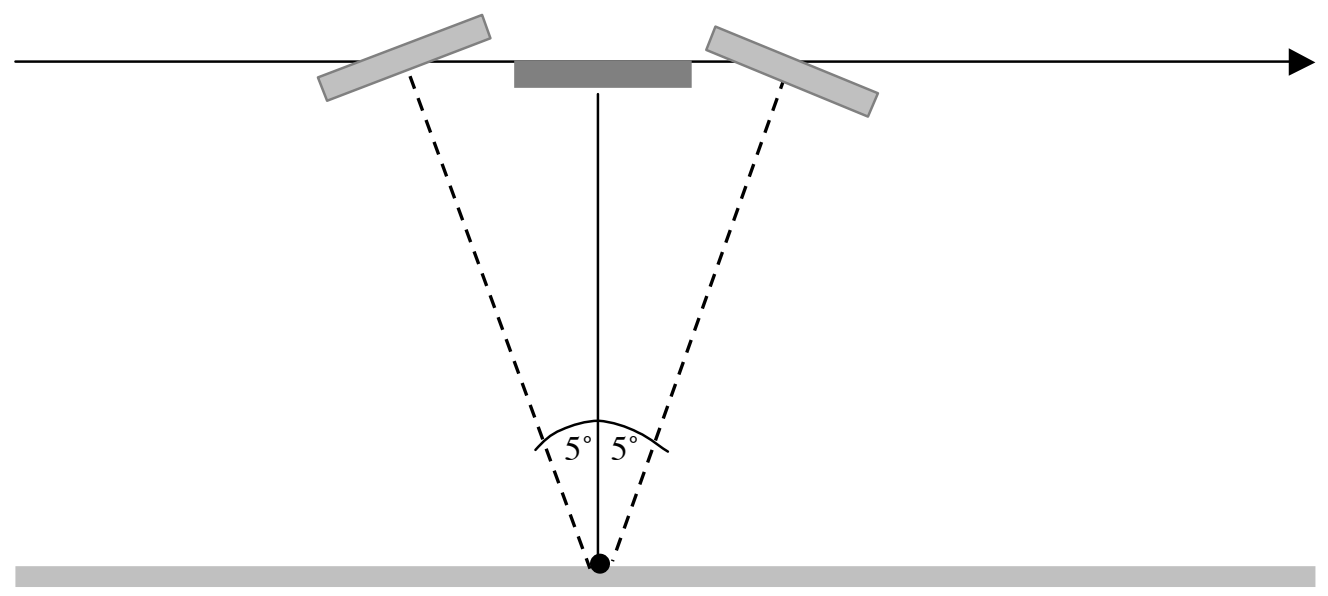

4) Derive the projection pattern for a 2-D rectangular aperture of length $L$ and width $W$ given in equation (A8).

5) What is the ground-range resolution of side-looking radar with a pulse length of $6 \times 10^{-8} \mathrm{~s}$ and a look angle of $45^{\circ}$ ?

7) (a) What is the period for a satellite in a circular orbit about the moon where the radius of the orbit is $1.9 \times 10^{6} \mathrm{~m}$ ? The mass of the moon is $7.34 \times 10^{22} \mathrm{~kg}$.

(b) You are developing a SAR mission for the moon. The length of your SAR antenna is $10 \mathrm{~m}$. What minimum pulse repetition frequency is needed to form a complete aperture? The circumference of the moon is $1.1 \times 10^{7} \mathrm{~m}$. You will need the orbital period from problem (a).

8) Derive equation (A10)

9) A SAR with a $10 \mathrm{~m}$ long antenna is orbiting the earth with a ground speed of $7000 \mathrm{~ms}^{-1}$, What is the maximum possible swath width. Use a look angle to the near range of $45^{\circ}$ and assume the earth is flat. 
9) CryoSat is a nadir-looking altimeter that uses a burst-mode synthetic aperture to reduce the length of the radar footprint in the along-track direction. The radar has a pulse repetition frequency of $18 \mathrm{kHz}$ and uses 64 echoes in one burst to form the synthetic aperture. What is the along-track resolution of the footprint? What is the Fresnel distance for this configuration (ground track velocity $7000 \mathrm{~ms}^{-1}, 700 \mathrm{~km}$ altitude) 
Appendix B - SAR Image Formation

Overview of the range-doppler algorithm [Curlander and McDonough, 1991]

The basic SAR theory is conceptually simple (Appendix A) yet when one looks into the inner workings of the SAR processor it appears quite complicated. The most difficult part of the problem is related to the coupling between the azimuthal compression and the orbital parameters. In the old days when the satellite orbits were not known very accurately, one would use techniques such as clutterlock and autofocus to derive the orbital parameters from the data. This is no longer necessary with the high accuracy orbits available today. A standard processing sequence follows where the first two steps are done onboard the satellite while the remaining 4 steps are done by the user with a digital SAR processor. The discussion provided here is for the ERS-1 satellite although the concepts translate to any modern strip-mode SAR satellite.

\section{Processing onboard the satellite}

Demodulate - The electromagnetic wave consists of a chirp of bandwidth $B(\sim 15 \mathrm{MHz})$ superimposed on carrier frequency $\omega_{\mathrm{o}}(\sim 5 \mathrm{GHz})$. To record the return signal, one could simply digitize the amplitude of the electric field $E(t)$ at a sampling rate of $10 \mathrm{GHz}$ using 1-byte encoding. However, this would generate 10 Gbytes of data every second! We are really only interested in how the chirp has been delayed and distorted by the reflection off the Earth's surface. The trick here is to use the shift theorem [Bracewell, 1978, p. 108] to isolate the 15 $\mathrm{MHz}$ part of the spectrum containing the chirp. Suppose we multiply the signal $E(t)$ by $e^{i 2 \pi \omega_{o} t}$. This will shift the Fourier transform of the signal as shown in Figure B1.

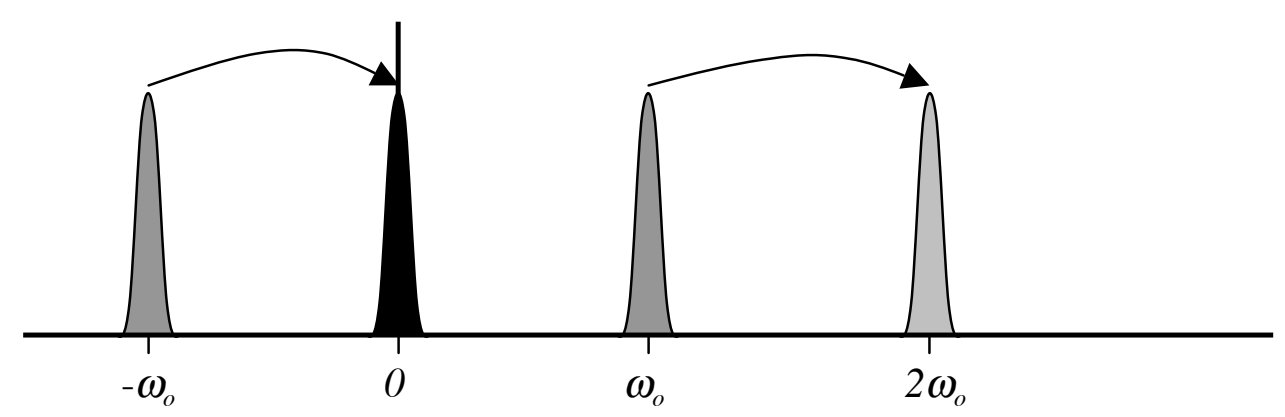

Figure B1. Diagram showing the power spectrum of the radar signal before and after shiting by the carrier frequency. 
The function $E(t)$ is a real-valued function so we know that its Fourier transform has Hermitian symmetry about the origin, that is $E(-\omega)=E^{*}(\omega)$. Because of this symmetry, all of the information in the time series is contained within the bandwidth of a single spectral peak. Next we low-pass filter the signal to isolate the spectral peak centered at the zero frequency. The original signal $E(t)$ was a real-valued function but after this shift/filter operation the output complex. No information is lost in this process and now the output can be digitized at the much lower rate of twice the bandwidth of the chirp $(\sim 30 \mathrm{MHz})$.

Digitize - The complex signal is digitized at 5 bits per pixel so the numbers range from 0 to 31 ; the mean value is 15.5. Digitizing the data at 8 bits per pixel would seem more convenient but transmitting these extra 3 bits to the ground receiving station exceeds the bandwidth of the telemetry so every effort is made to compress the data before transmission. Once the data are on the ground they are expanded to 8 bits (one byte) for programming convenience. Engineers refer to the real and imaginary parts of the signal as the in-phase (I) and quadrature (Q) components. The raw signal files contain rows of (11644 bytes) that represent a single radar echo (Figure B2). The first 412 bytes are timing and other information while the remaining 11232 bytes contain the 5616 complex numbers of raw signal data.

Figure B2. Raw signal data

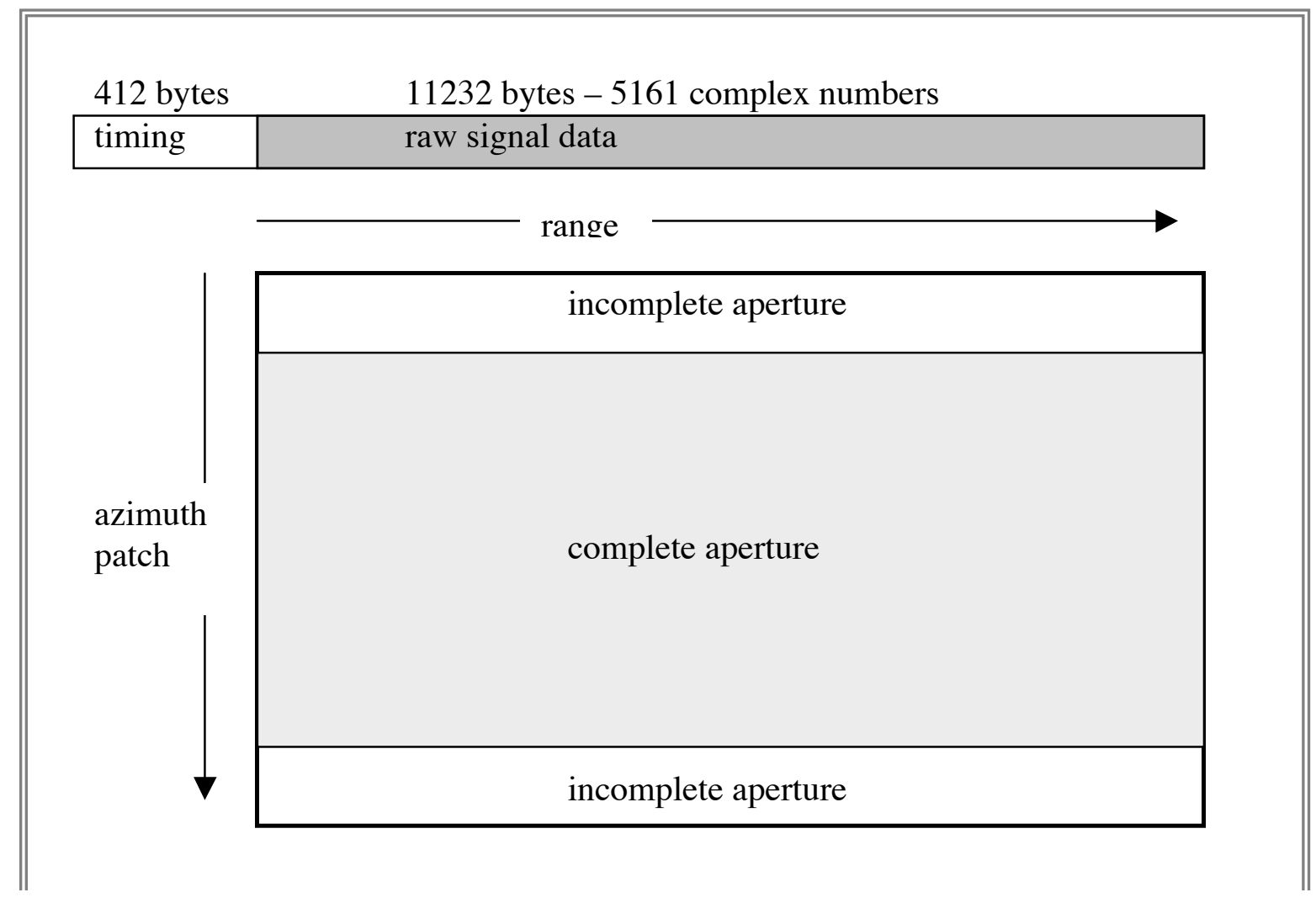




\section{Digital SAR processing}

The digital SAR processor is a computer program that converts the raw signal data into a single-look complex (SLC) image. An overview is provided in Figure B3 this is followed by a detailed description of each step.

Figure B3. Digital SAR processing overview

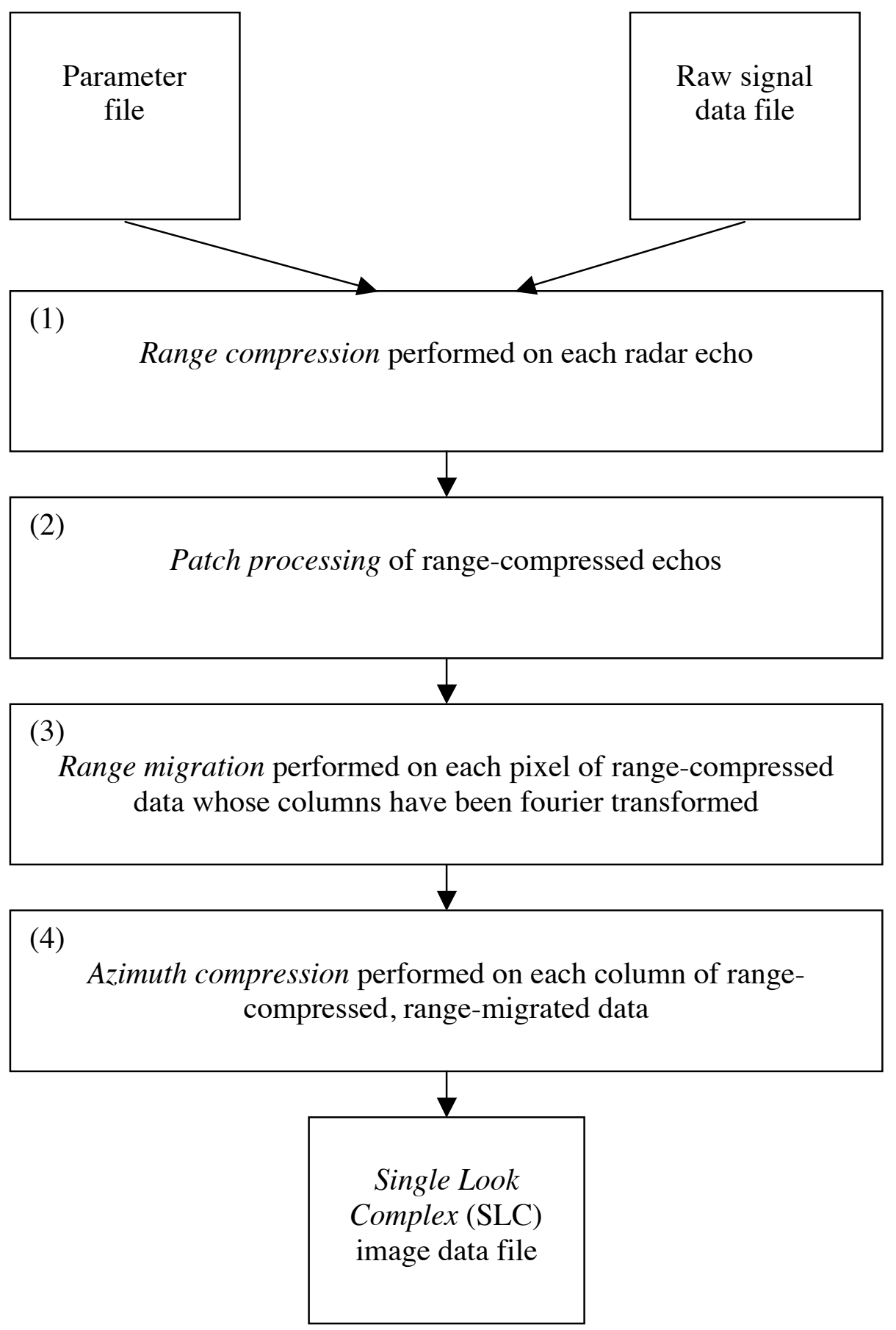


Table B1.Example Parameter (PRM) file (e2_10001_2925.PRM)

\section{file information/format}

input_file

SC_identity

bytes_per_line

first_sample

fd1

I_mean

Q_mean

icu_start

SC_clock_start

SC_clock_stop e2_10001_2925.fix

2

11644

206

248.115

15.5

15.5

2576039268.848

1997078.767881851

1997078.768074699

name of raw data file

(1) - ERS-1 or (2) - ERS-2

number of bytes in row of raw data

412 bytes of timing information to skip over

Doppler centroid estimated from data $\quad f_{D c}$

mean value of real numbers

mean value of imaginary numbers

spacecraft clock for first sample

UTC for first sample YYYDDD.DDDDDDDD

UTC for last sample YYYDDD.DDDDDDDD

radar characteristics

PRF

rng_samp_rate

chirp_slope

pulse_dur

radar_wavelength
1679.902394

$1.89625 \mathrm{e}+07$

$4.17788 \mathrm{e}+11$

$3.712 \mathrm{e}-05$

0.056666

\section{orbital information}

near_range

earth_radius

SC_height

SC_vel
829924.365777

6371746.4379

787955.52

7125.0330 pulse repitition frequency

range sampling rate

chirp slope

pulse duration

radar wavelength

distance to first range bin

earth radius $\sim 1 / 2$ way into image

spacecraft height above the earth_radius

effective speed
PRF

$f_{s}$

$k$

$\tau_{p}$

$\lambda$

$R_{\text {near }}$

$R_{e}$

$H$

\section{processing information}

$\begin{array}{ll}\text { num_valid_az } & 2800 \\ \text { num_patches } & 10 \\ \text { first_line } & 1 \\ \text { deskew } & \mathrm{n} \\ \text { st_rng_bin } & 1 \\ \text { num_rng_bins } & 6144 \\ \text { chirp_ext } & 614 \\ \text { Flip_iq } & \mathrm{n} \\ \text { nlooks } & 1\end{array}$

size of patch to process

number of patches to process

deskew (yes or no)

start processing at row number

number of range bins in output file

extend the chirp to process outside swath

exchange real and imaginary numbers (yes or no)

number of azimuth echoes to average

\section{image alignment}

rshift

15.1

ashift

483.2

stretch_r

.0014569

stretch_a

$-.0019436$

a_stretch_r

0.0

a_stretch_a

0.0

range shift to align image to master

azimuth shift to align image to master

range stretch versus range

azimuth stretch versus range

range stretch versus azimuth

azimuth stretch versus azimuth 
(1) Range Compression - A sharp radar pulse is recovered by deconvolution of the chirp. This is done with fast fourier transform. There are 5616 points in the ERS-1 signal data and the chirp is 703 points long. Both are zero-padded to a length of 8192 prior to deconvolution to take advantage of the speed of the radix- $2 \mathrm{fft}$ algorithms. Later we keep 6144 points, which enables one to extract the phase beyond the edges of the original swath. Since the chirp really did interact with the ground outside the digitized swath, this is not magic. The number 6144 has small prime factors that may be optimal for later 2-D fft phase unwrapping algorithms.

(2) Patch Processing - The next step is to focus the image in the along-track or azimuth direction. This is also done by fft, but now we need to process columns rather than rows. For the ERS radar, the synthetic aperture is 1296 points long so we need to read at least that many rows into the memory of the computer. Actually 4096 rows is better number since it is a power of 2. A typical ERS raw data file has 28,000 rows so we will need to process many of these patches. One problem with this approach is that the synthetic aperture is only complete for the inner 2800 points of the 4096 data patch so the patches must have a 1296-point overlap (Figure B3). A 4096 x 5616 patch requires at least 184 Mbytes of computer memory for single precision real numbers. In the old days before this large memory space was available, each patch was transposed - corner turning using high-speed disks so the data could be processed on a column-by-column basis. The L-band data from ALOS have a much longer synthetic aperture and patch size. Efficient processing of these data require a minimum of 2 Gbytes of computer memory.

(3) Range Migration - As we will see below, a point target will appear as a hyperbolicshaped reflection as it moves through the synthetic aperture. In addition, there could be a pronounced linear drift due to an elliptical orbit and earth rotation. In other words, the target will migrate in range cell as a linear trend plus a hyperbola. The shape of this migration path is calculated from the precise orbital information and is approximated by a parabola. Prior to focusing the image along a single column, these signals must be migrated back to a constant range cell. This is called range migration and the fastest way 
to do this is by Fourier transforming the columns first. Each Fourier component corresponds to a unique Doppler shift and also a unique value of range migration; For an ideal radar, the first component will have zero Doppler and will correspond to the point on the earth that is perpendicular to the spacecraft velocity vector. The second component will have a small positive Doppler shift and a small migration of the range cells will be needed - and so on all the way through the positive and negative Doppler spectrum.

(4) Azimuth Compression - The final step in the processing is to focus the data in azimuth by accounting for the phase shift of the target as it moves through the aperture. In Appendix A, we calculated the illumination pattern on a screen due to the propagation of a coherent wave from the aperture to the screen. The azimuth compression relies on the same theory although we do the opposite calculation - given the illumination pattern, calculate the shape of the aperture (reflectivity of the target). This is done by generating a second frequency-modulated chirp where the chirp parameters depend on the velocity of the spacecraft, the pulse repetition frequency $(P R F)$, and the absolute range. The chirp is Fourier transformed into Doppler space and multiplied by each column of range-migrated data. The product is inverse Fourier transformed to provide the focused image.

Single-Look Complex (SLC) Image - As described above, only the inner 2800 rows use the complete synthetic aperture so these are written to an output file (Figure B2). The file-reading pointer is moved back 1296 rows to start the processing of the next patch. Because complete apertures are used for each patch, they will abut seamlessly.

\section{Range compression}

To reduce the peak power of the radar transmitter associated with a short pulse, a long frequency-modulated chirp is emitted by the radar. This chirp propagates to the ground where it reflects from a swath typically $100 \mathrm{~km}$ wide. When it returns to the radar, the raw signal data consists of the complex reflectivity of the surface convolved with the chirp. Our objective is to recover the complex reflectivity by deconvolution of the chirp. As discussed above, this is the first step in the digital SAR processor. For the ERS-radar, the frequency-modulated chirp is 
$s(t)=e^{i \pi k t^{2}} \quad|t|<\tau_{p} / 2$

where

$k \quad$ - chirp slope $\left(4.17788 \times 10^{11} \mathrm{~s}^{-2}\right)$

$\tau_{p} \quad$ - pulse duration $\left(3.712 \times 10^{-5} \mathrm{~s}\right.$ or $\sim 11 \mathrm{~km}$ long $)$

$f_{s} \quad$ - range sampling rate $\left(1.89625 \times 10^{7} \mathrm{~s}^{-1}\right)$.

An example of a portion of the chirp for the ERS- radar as well as its power spectrum and impulse response is shown below.
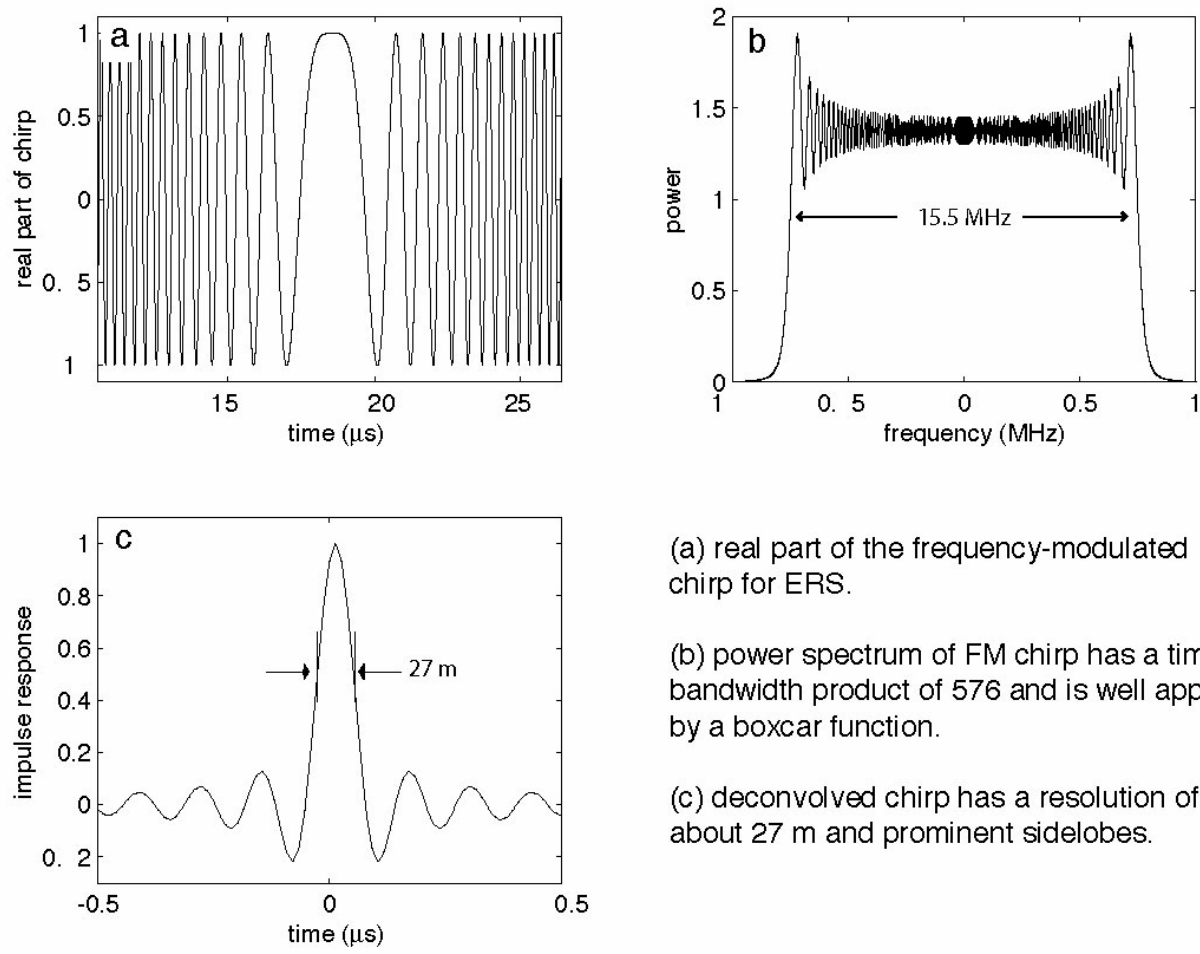

(a) real part of the frequency-modulated chirp for ERS.

(b) power spectrum of FM chirp has a timebandwidth product of 576 and is well approximated by a boxcar function.

(c) deconvolved chirp has a resolution of about $27 \mathrm{~m}$ and prominent sidelobes.

Figure B4 Chirp for ERS-1

The bandwidth of the chirp, $B=k \tau_{p}$, has a value of $15.5 \mathrm{MHz}$ for the ERS radar. A matched filter is used to deconvolve the chirp from the data. In this case, the matched filter is simply the complex conjugate of the chirp or $s^{*}(t)=e^{-i \pi k t^{2}}$. The convolution of $s^{*}(t)$ and $s(t)$ is shown in Figure B4b. The effective range resolution of the radar is about $27 \mathrm{~m}$. 
Using computer code and the radar parameters provided above, one can deconvolve the ERS chirp. The figure below is a small patch of ERS-2 data for an area around Pinon Flat, California (track 127, frame 2925, orbit 10001). This area contains two radar reflectors (Figure B5 and Table B2) spaced about $600 \mathrm{~m}$ apart that were installed in late 1996. The amplitude of the raw signal data appears as noise with a single horizontal streak due to missing data (Figure B5 - left). The range-compressed data (right image) shows two vertical streaks due to the high reflectivity of the reflectors (red circles) as they migrate through the synthetic aperture.
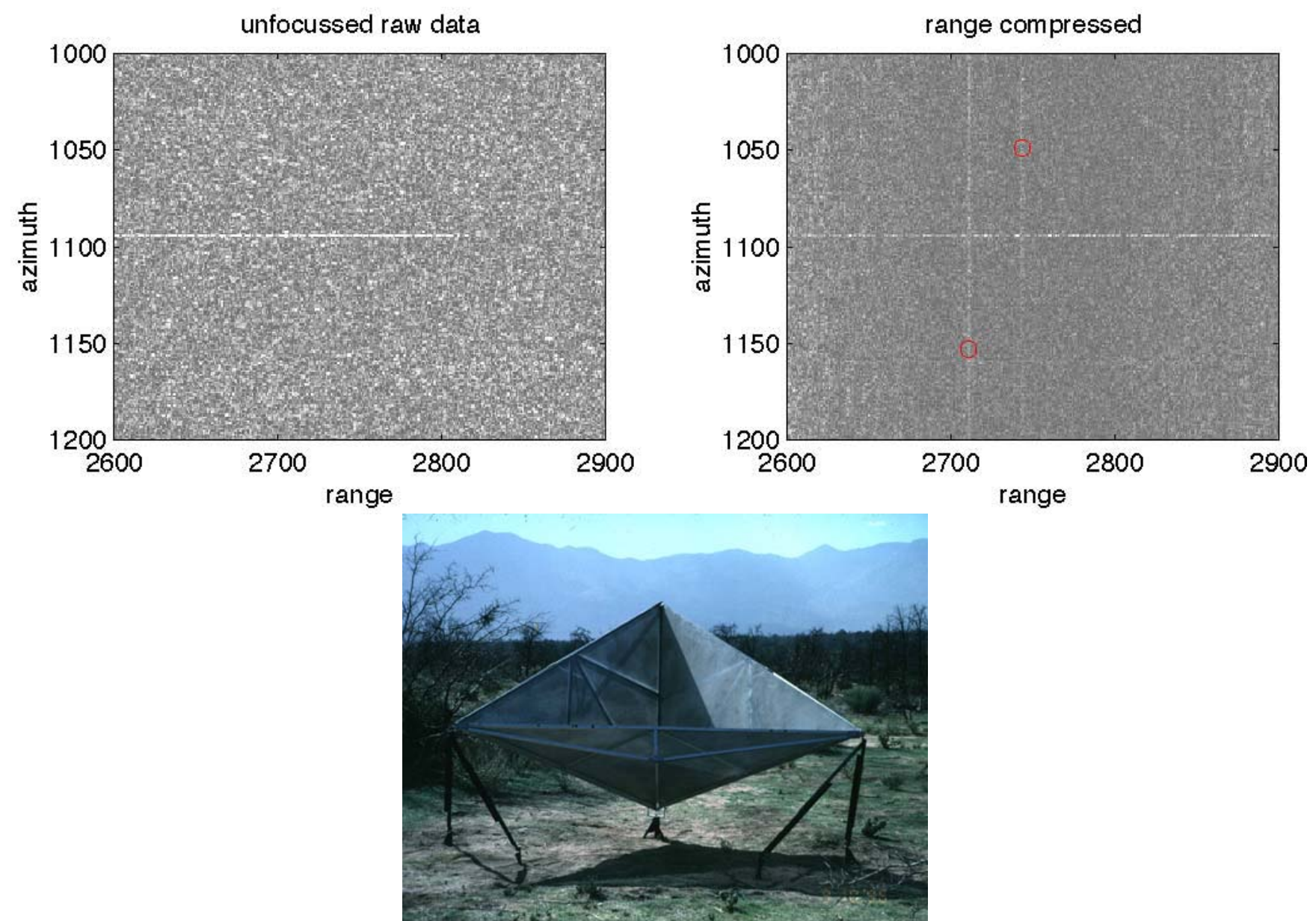

Figure B5. Raw ERS signal data (left) and range compressed data (right) for data over Pinon Flat California where two radar corner reflectors are installed (lower). 
Table B2. Coordinates of Radar Reflectors

\begin{tabular}{|c|c|c|c|c|}
\hline \multicolumn{3}{|l|}{ position } & \multicolumn{2}{|c|}{ orientation } \\
\hline lat & Ion & height & elev. & azi. \\
\hline 33.612246 & -116.456768 & 1258.990 & $39^{\circ}$ & $257.5^{\circ}$ \\
\hline 33.612253 & -116.457893 & 1257.544 & $39^{\circ}$ & $102.5^{\circ}$ \\
\hline 33.607373 & -116.451836 & 1254.537 & $39^{\circ}$ & $102.5^{\circ}$ \\
\hline
\end{tabular}

Latitude and longitude in decimal degrees and height in meters relative to the WGS-84 co-ordinate system and ellipsoid. The survey point is the apex (lowest corner) of each reflector. There should be a correction for the offset between the phase center of the reflector and the apex.

\section{Azimuth compression}

Azimuth compression or azimuth focusing involves coherent summation of echos at a constant range from the point reflector. The geometry of the strip-mode acquisition is shown in Figure B6

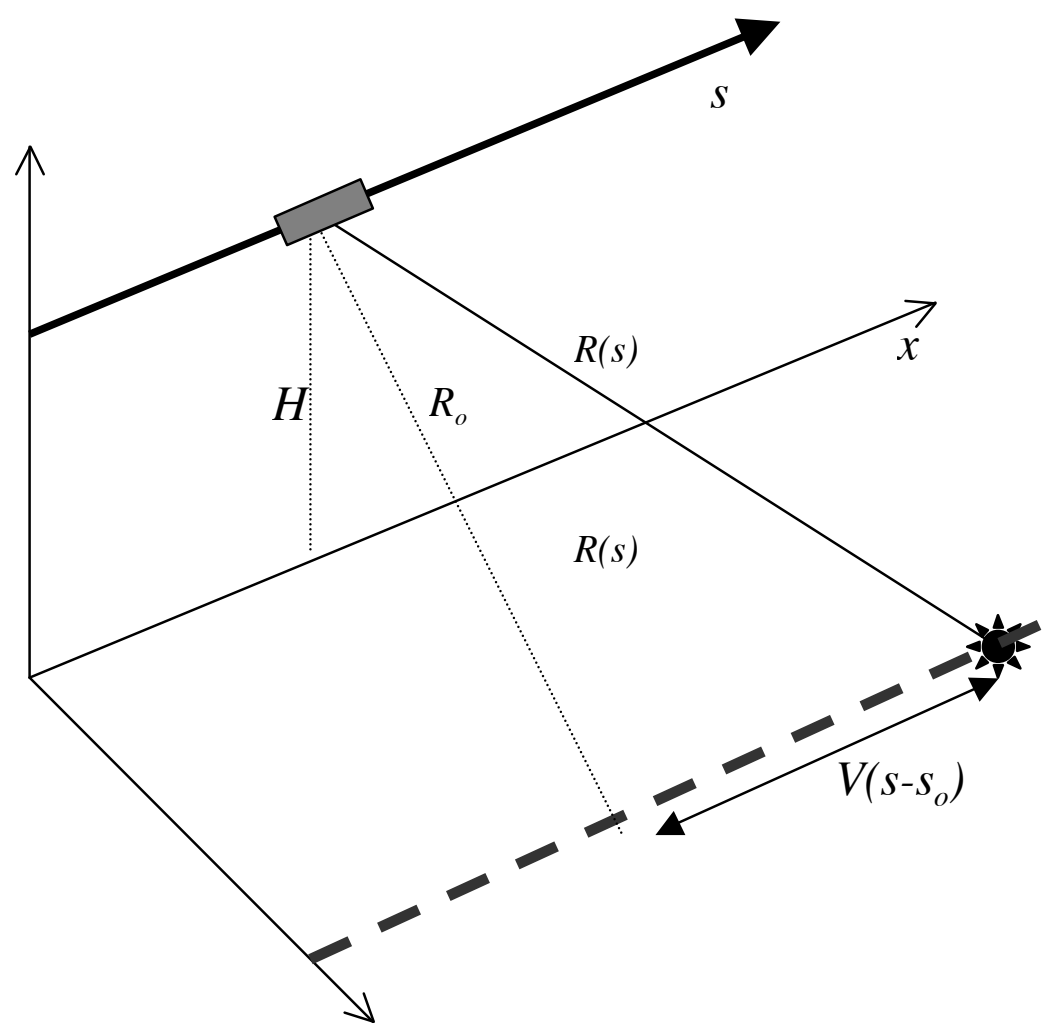

Figure B6. Geometry of radar passing over a point reflector where

$V \quad-$ the effective speed which is about equal to the ground track speed

$s \quad-$ slow time along the satellite track

$S_{o} \quad-$ time when the center of the radar echo passes over the point reflector

$R_{o}=R_{\text {near }}+n *(C / f s)$ minimum range from the spacecraft to the target

$R_{\text {near }} \quad-$ near range to first data sample in the swath 
The range to the point reflector evolves with time as

$$
R^{2}(s)=R_{o}^{2}+V^{2}\left(s-s_{o}\right)^{2}
$$

The complex phase of the return echo is

$C(s)=\exp \left[-i \frac{4 \pi}{\lambda} R(s)\right]$

The range versus slow time is approximately a hyperbola but for mathematical convenience we'll approximate this using a parabola

$R(s)=R_{o}+\dot{R}_{o}\left(s-s_{o}\right)+\frac{\ddot{R}_{o}}{2}\left(s-s_{o}\right)^{2}+\ldots$

where the dot indicates derivative with respect to slow time, s. Curlander and McDonough [1991] discuss the accuracy of this polynomial approximation and it is also discussed below in terms of the ALOS SAR. The approximation is good enough for strip-mode SAR but may be inadequate for the much longer apertures associated with spotlight-mode SAR. Now we can write the phase of the return signal as a function range, range rate, and range acceleration.

$C(s)=\exp \left\{-i \frac{4 \pi}{\lambda}\left[R_{o}+\dot{R}_{o}\left(s-s_{o}\right)+\ddot{R}_{o}\left(s-s_{o}\right)^{2} / 2\right]\right\}$

It is more common to describe the parameters for focusing the SAR image as the Doppler centroid $f_{D c}$ and the Doppler frequency rate $f_{R}$. The relationships are:

$f_{D c}=\frac{-2 \dot{R}}{\lambda}$ and $f_{R}=\frac{-2 \ddot{R}}{\lambda}$ 
or

$C(s)=\exp \left\{-i \frac{4 \pi R_{o}}{\lambda}\right\} \exp \left\{i 2 \pi\left[f_{D c}\left(s-s_{o}\right)+f_{R}\left(s-s_{o}\right)^{2} / 2\right]\right\}$

Note that this function is another frequency-modulated chirp where the parameters are the Doppler centroid and the Doppler frequency rate. An example of this azimuthal chirp function for the ERS orbit/radar as well as its power spectrum and impulse response are shown in Figure B7.
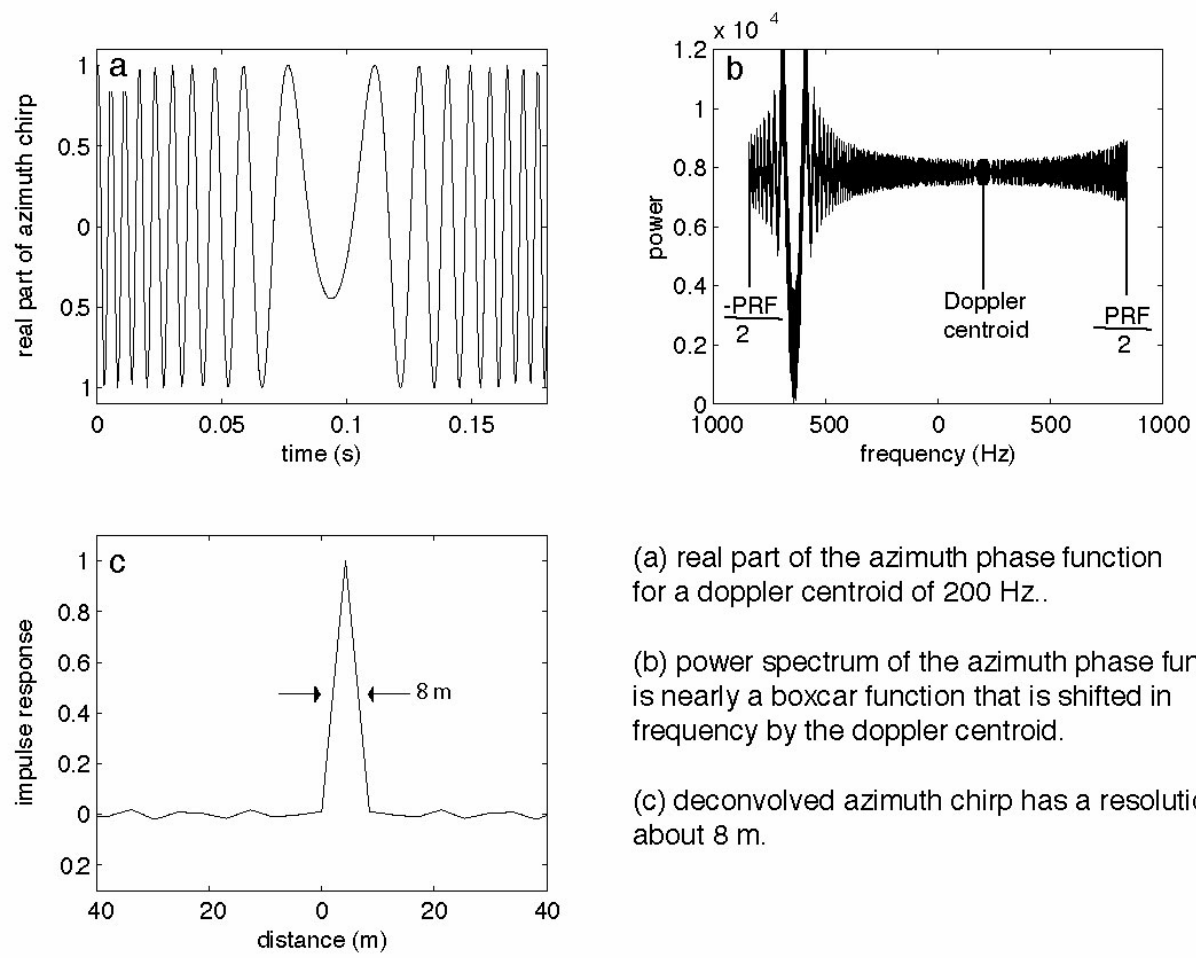

(a) real part of the azimuth phase function for a doppler centroid of $200 \mathrm{~Hz}$.

(b) power spectrum of the azimuth phase function is nearly a boxcar function that is shifted in frequency by the doppler centroid.

(c) deconvolved azimuth chirp has a resolution of about $8 \mathrm{~m}$.

Figure B7. Azimuthal phase for the ERS-1 spacacraft.

The bandwidth of the azimuthal chirp is equal to the pulse repetition frequency (PRF $=1680$ $\mathrm{Hz}$ ). In this case the matched filter is simply the complex conjugate of the azimuthal phase function (B7) or $C^{*}(s)$.

Doppler Centroid, Doppler Rate, and Effective Speed - Proper focus of the image requires accurate estimates of the three parameters given in (B5) and (B7). Actually we only need to 
estimate two parameters because we will sweep through the range cells $n$ in the raw data file and focus the image at each range $R_{o}=R_{\text {near }}+n *\left(C / f_{s}\right)$ where $C$ is the speed of light and $f_{s}$ is the range sampling rate. Ideally the beam of the radar would be steered so that it is centered at a point on the ground where the range is minimum so the Doppler centroid is zero or at least small. We follow the approach of Masden [1989] and calculate the Doppler centroid directly from the raw data file by computing the average change in phase between adjacent rows of the raw data file. This assumes that the Doppler centroid does not vary with slow time although it could vary slightly with increasing range. We use this estimated Doppler centroid to focus the image (or select a similar Doppler centroid that is closes to the mean of a stack of repeated SAR images).

Finally, as discussed below, the precise orbit can be used to calculate the third parameter, the range acceleration $\ddot{R}$. As shown in Figure B7, this parameter should decrease with increasing range. One approach would be to use the precise orbit to calculate range acceleration for each range but this is unnecessary because there is a good formula for range acceleration as a function of range that involves a parameter called the effective speed $V$ of the spacecraft. Indeed the SAR processor code takes effective speed and converts this to $\ddot{R}$ to focus the image. The derivation of the effective speed parameter follows. We start by taking the first derivative of the range versus slow time given in (B2)

$\frac{\partial R^{2}(s)}{\partial s}=2 R \dot{R}=2 V^{2} s$

The second derivative is

$$
\dot{R} \dot{R}+R \ddot{R}=V^{2} .
$$

Next we assume that the radar antenna is oriented nearly perpendicular to the velocity vector of the satellite so $\dot{R}<<$. Note that to achieve interferometric coherence the radar beams of the reference and repeat images must overlap spatially which essentially requires that the Doppler centroid be less than half of the pulse repitition frequency (PRF). Given a typical PRF for a spaceborne SAR of 2000 and using equation (B6) and a wavelength of $0.05 \mathrm{~m}$ the range rate must be less than $\sim 25 \mathrm{~m} / \mathrm{s}$. This is much less than the typical spacecraft velocity of $7800 \mathrm{~m} / \mathrm{s}$ so 
we can safely ignore the first term on the left hand side of (B9). So the approach is to use the precise orbit to calculate the range acceleration $\ddot{R}$ at a single range $R_{o}$ within the image. These two numbers are used to calculate the effective speed

$V=\sqrt{R_{o} \ddot{R}}$

Finally, this parameter is used by the SAR processing code to calculate the range acceleration

$\ddot{R}=V^{2} / R$

at all the other ranges in the swath.

The effective speed has a physical interpretation as the ground track speed of the spacecraft. For a non-rotating spherical earth of radius $R_{e}$ the ground track speed is

$$
V=\frac{V_{s}}{\left(1+\frac{H}{R_{e}}\right)^{1 / 2}}
$$

where $V_{s}$ is the speed of the satellite at an orbital altitude of $H$. For C-band radars such as ERS and ENVISAT the effective speed calculated from (B10) provides adequate focus for the SAR image. However, for L-band SARs such as ALOS, the aperture is much longer so other factors must be considered such as the curvature and ellipticity of the orbit as well as the rotation rate of the Earth. We bypass this complexity by computing the effective speed from a polynomial fit of range versus time as discussed below but first we discuss the overlength of the synthetic aperture.

Length of synthetic aperture - The length of the synthetic aperture $L_{a}$ depends on the length of the radar ground footprint in the azimuth direction, which is approximately

$$
L_{a}=R_{o} \frac{\lambda}{L}
$$


where $L$ is the physical length of the antenna, $\lambda$ is the wavelength and $R_{o}$ is the slant range given above. The length of the aperture in terms of radar echos is given by

$n_{a}=\frac{L_{a} P R F}{V}$

This is simply the length of the synthetic aperture divided by the along-track sampling distance.

The following table provides these quantities for SARs of interest for interferometry today.

Table B3. Length of synthetic aperture for three satellites.

\begin{tabular}{|l|l|l|l|l|l|l|l|l|}
\hline & $\begin{array}{c}V \\
(\mathrm{~m} / \mathrm{s})\end{array}$ & $\begin{array}{c}\lambda \\
(\mathrm{m})\end{array}$ & $\begin{array}{c}P R F \\
(\mathrm{~Hz})\end{array}$ & $\begin{array}{c}R_{o} \\
(\mathrm{~km})\end{array}$ & $\begin{array}{c}L \\
(\mathrm{~m})\end{array}$ & $\begin{array}{c}L_{a} \\
(\mathrm{~m})\end{array}$ & $\begin{array}{c}n_{a} \\
\text { theory }\end{array}$ & $\begin{array}{c}n_{a} \\
\text { actual }\end{array}$ \\
\hline ERS-1/2 & 7125 & 0.057 & 1679 & $\sim 850$ & 10 & 4850 & 1142 & 1164 \\
\hline Envisat & 7125 & 0.057 & 2067 & $\sim 1020$ & 10 & 5830 & 1690 & 1740 \\
\hline ALOS & 7125 & 0.236 & 2159 & $\sim 1020$ & 9 & 26,830 & 8128 & 9216 \\
\hline
\end{tabular}

Focussed image - Using the azimuth compression formulas and parameters provided above, one can focus the image in the azimuth direction. The figures below show the same small patch of ERS-2 data for an area of Pinon Flat, California. The range-compressed data (left image) shows two vertical streaks due to the high reflectivity of the reflectors (red circles) as they migrate through the synthetic aperture. The fully focused image (right) shows the point-like reflectivity associated with the two radar reflectors.
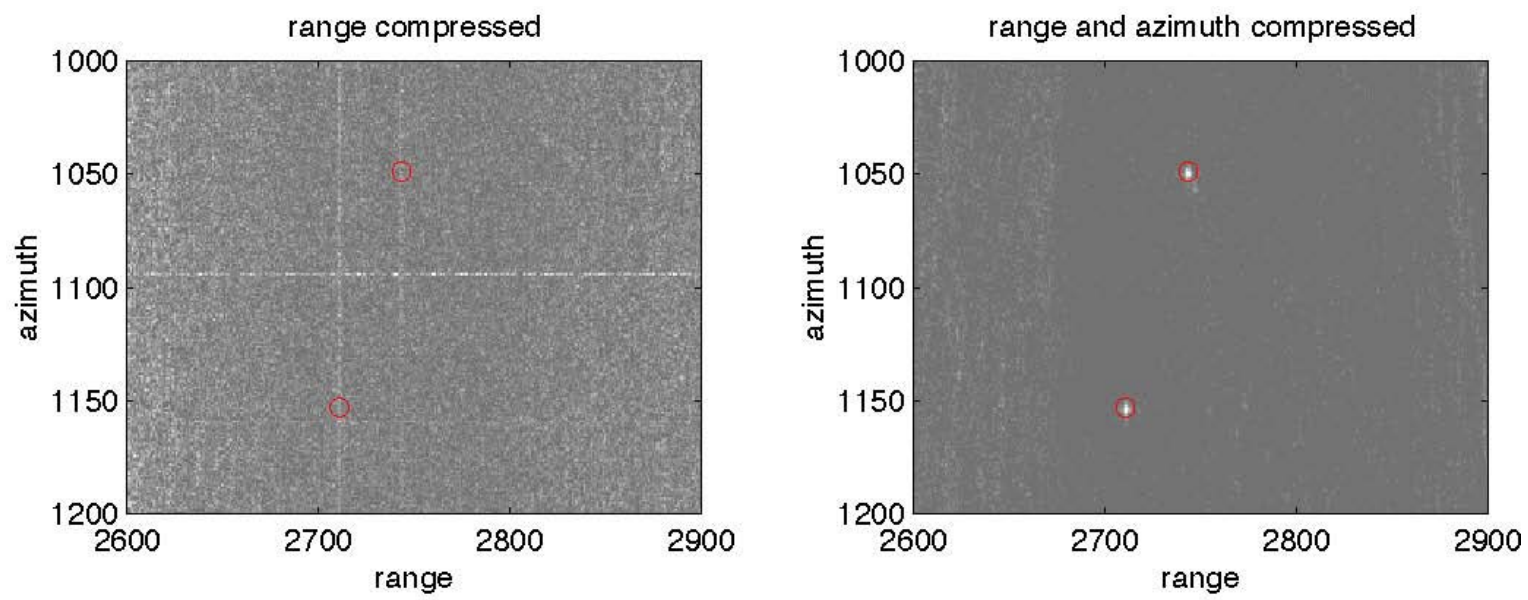

Figure B8. Range compressed (left) and fully focussed image (right). The two bright reflectors are 3-m corner cubes at Pinon Flat observatory. These were deployed in 1996 and therefore provide stable calibration points for ERS-1, ERS-2, Envisat, ALOS and all future satellites. 


\section{Estimating $\ddot{R}$ when a precise orbit is available}

Estimation of the range acceleration from the orbit seems conceptually trivial although there is one complication that requires a bit of geometry. The complication is to find a point on the surface of the earth, at a prescribed range, and convert that location to Cartesian coordinates. Consider the following three vectors which form a triangle;

$\vec{R}_{s}$ - the vector position of the satellite in the Earth-fixed coordinate system;

$\vec{R}_{e}$ - the vector position of a point scatterer on the Earth and somewhere in the SAR scene;

$\vec{R}_{o}$ - the line-of-sight vector between the satellite and the point scatterer.

The three vectors form a triangle such that $\vec{R}_{e}=\vec{R}_{s}+\vec{R}_{o}$ (Figure B9). The scalar range, which is a function of slow time, is given by

$$
R(s)=\left|\vec{R}_{s}(s)-\vec{R}_{e}\right| \cong R_{o}+\dot{R}_{o}\left(s-s_{o}\right)+\frac{\ddot{R}_{o}}{2}\left(s-s_{o}\right)^{2}+\ldots
$$

Measurements of scalar range versus slow time can be used to estimate the coefficients of the parabolic approximation. The algorithm is to:

(1) Use the precise orbit to calculate the position vector of the satellite and compute a time series $R(s)$ over the length of the aperture.

(2) Perform a least-squares parabolic fit to this time series to estimate $R_{o}, \dot{R}_{o}$, and $\ddot{R}_{o}$.

(3) Compute the effective speed as $V_{e}^{2}=R_{o} \ddot{R}_{o}$.

The only remaining step is to calculate the position of the point target $\vec{R}_{e}=\vec{R}_{s}+\vec{R}_{o}$. Actually this can be any point in the image so the selection criteria is that the point lie at the proper radius of the surface of the Earth $R_{e}=\left|\vec{R}_{e}\right|$ and the $\vec{R}_{o}$ vector is perpendicular to the velocity vector of the satellite $\vec{V}$. If we prescribe the length of the $R_{o}$ - vector then the angle between the satellite position vector and the line-of-sight vector is given by the law of cosines as shown in Figure B9. 


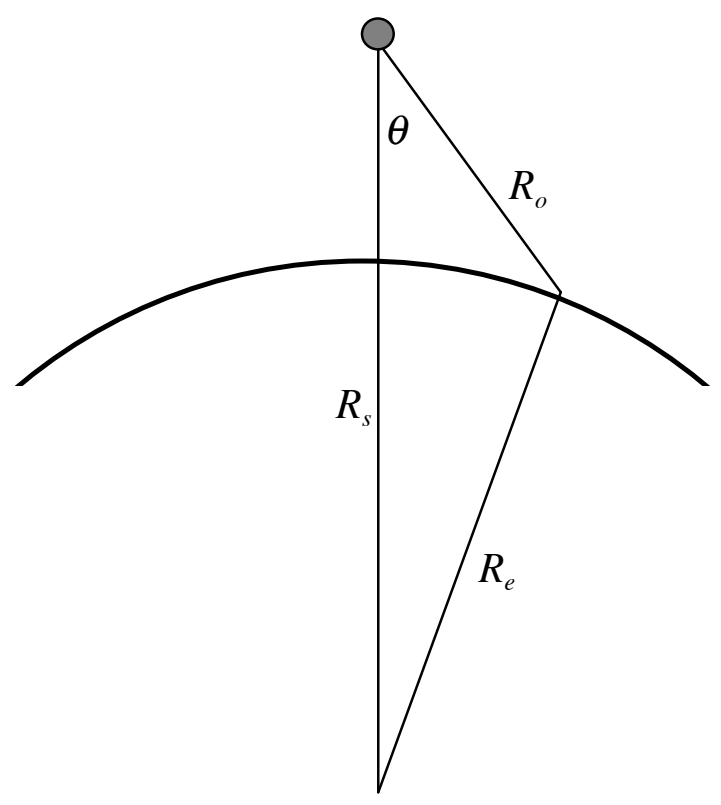

Figure B9. Triangle of earth radius, satellite radius and range to a point on the surface of the earth used to compute look angle $\theta$.

This diagram has the satellite at the gray dot with the velocity vector pointing into the page. The cosine of the look angle is

$\cos \theta=\frac{\left(R_{s}^{2}+R_{o}^{2}-R_{e}^{2}\right)}{2 R_{s} R_{o}}$

Next consider a local co-ordinate system where the $q_{2}$ axis is aligned with the velocity vector of the satellite as shown in the following diagram. 


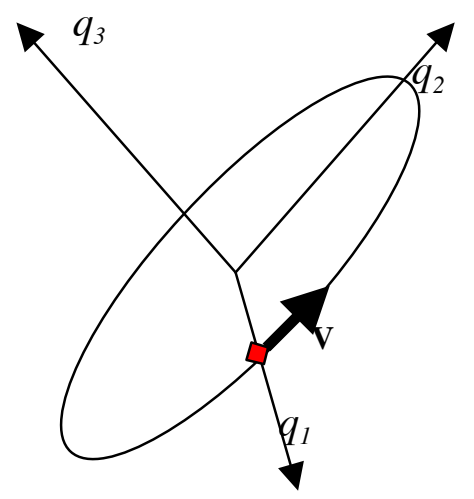

Figure B10. Satellite (red) in circular inclined orbit about the Earth.

In this coordinate system, the $q_{1}$-axis is parallel to $\vec{R}_{s}$, the $q_{2}$ axis is parallel to $\vec{V}$, and the $q_{3}$ axis is perpendicular to both and is given by their cross product

$q_{3}=\frac{\vec{R}_{s}}{\left|R_{s}\right|} \times \frac{\vec{V}}{|\vec{V}|}$

The following diagram has the velocity vector of the satellite going into the page and aligned with the $q_{2}$ coordinate. The $q_{1}$ vector is the radial vector from the center of the earth. The lineof-sight right-look vector is in the $q_{1}-q_{3}$ plane as shown in the diagram.

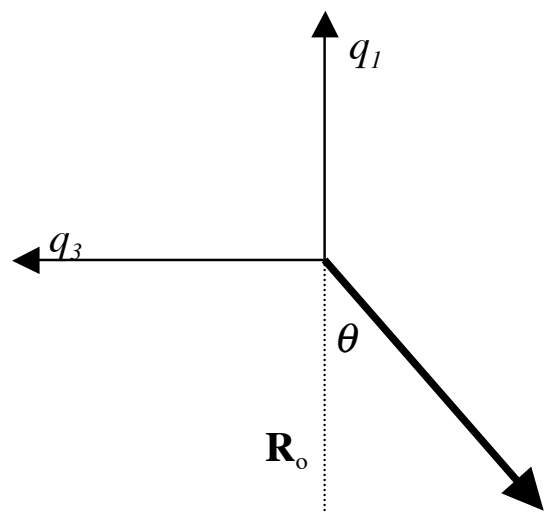

Figure B11. Diagram of satellite-fixed coordinate system where $q_{1}$ is the vector from the center of the earth to the spacecraft, $q_{2}$ is parallel to the velocity vector of the SC. The bold vector is the look vector of the radar where $\theta$ is the look angle. 
After going through this geometry we find the line of sight (LOS) vector to a point on the surface of the earth at the prescribed range is

$\vec{R}_{o}=R_{o}\left(\begin{array}{lll}-\cos \theta \hat{q}_{1} & 0 \hat{q}_{2} & -\sin \theta \hat{q}_{3}\end{array}\right)$

\section{Example with ALOS L-band orbit}

Now that we have an algorithm for finding a point on the surface of the Earth that is within the SAR scene, we can compute the time-evolution of the range to that point as the satellite orbits above the rotating Earth. We consider data from a descending orbit over Koga Japan where three radar reflectors have been deployed. The image is a fine-beam, single polarization having a nominal look angle of 34.3 degrees. A Hermite polynomial interpolation was used to calculate the $x-y-z$ position of the satellite from 28 position and velocity vectors spaced at 60 second intervals. Thus the entire arc is 28 minutes or a quarter on an orbit. The accuracy of the Hermite interpolator was checked by omitting a central point and performing an interpolation using 6 surrounding points. The accuracy of the interpolation was found to be better than 0.2 $\mathrm{mm}$ suggesting that the 60-s interval provides an accurate representation of the orbital arc.

Next we compute the range to the ground point as a function of time before and after the

perpendicular LOS vector $\vec{R}_{o}$. We used a before/after time interval of 3 seconds which is about twice the length of the synthetic aperture for ALOS. A second-order polynomial was fit to the range versus time function and the three coefficients provide estimates of $R_{o}, \dot{R}_{o}$, and $\ddot{R}_{o}$. The range versus time, as well as the residual of the fit, are shown below. One can learn a great deal from this exercise. 

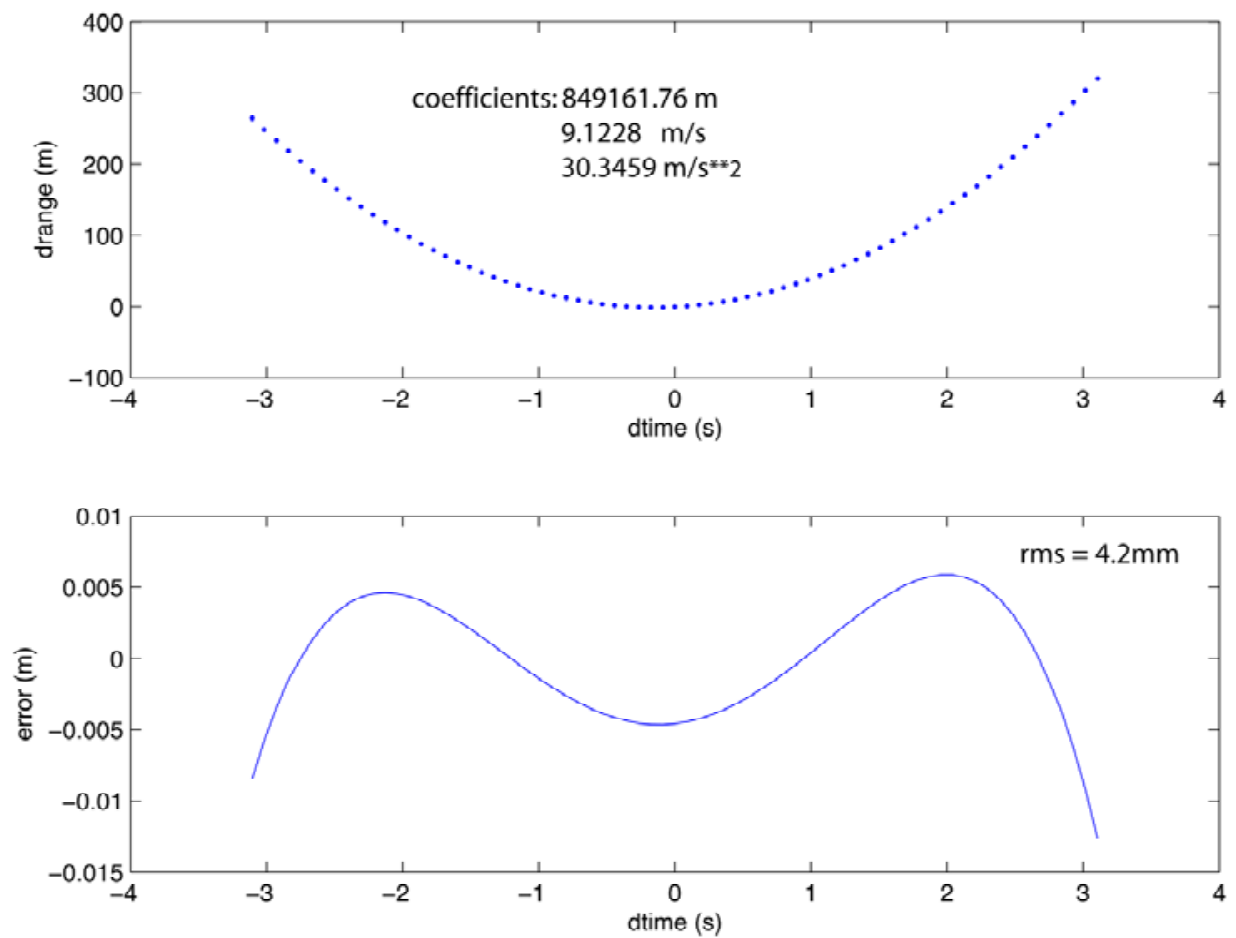

Figure B12. (upper) range versus slow time for ALOS for a 6 second aperture. The actual aperture is 4.2 seconds (Table B3). (lower) deviation from a parabolic fit to this range vs. time function.

1) The first thing learned is that the parabolic approximation to a hyperbola used in the SAR processor has a maximum error of about $1 \mathrm{~cm}$ at a time offset of 3 seconds. This corresponds to a small fraction of the $23-\mathrm{cm}$ wavelength. Note that the actual aperture length for ALOS is only +/- 2.1 seconds so this approximation is justified.

2) The range at zero offset has an error of $-0.24 \mathrm{~m}$. This is due to approximating the shape of the earth as a sphere having a local radius given by the WGS84 ellipsoid formula. In other words there is a small error in the LOS vector because it intersects the surface of the Earth at a latitude that is slightly different from the spacecraft latitude so the earth radii will differ slightly.

3) The Doppler shift when the satellite is perpendicular to the target can be calculated from the range rate coefficient using equation (B6).

4) Finally the range acceleration can be used to calculate the effective speed of the satellite $V_{e}^{2}=R_{o} \ddot{R}_{o}$. For this example we arrive at a speed of $7174 \mathrm{~m} / \mathrm{s}$. By trial and error we found the optimal focus parameter corresponds to a speed between 7173 and 7183 so this new estimate is 
accurate. The simple spherical orbit ground speed approximation (B12) yields an estimate of $7208 \mathrm{~m} / \mathrm{s}$ which provides poor focus.

Squint angle - Finally lets examine the quantity, $\frac{V\left(s-s_{o}\right)}{R_{o}}$. This is related to something called the squint angle, $\gamma$, as shown in the following diagram.

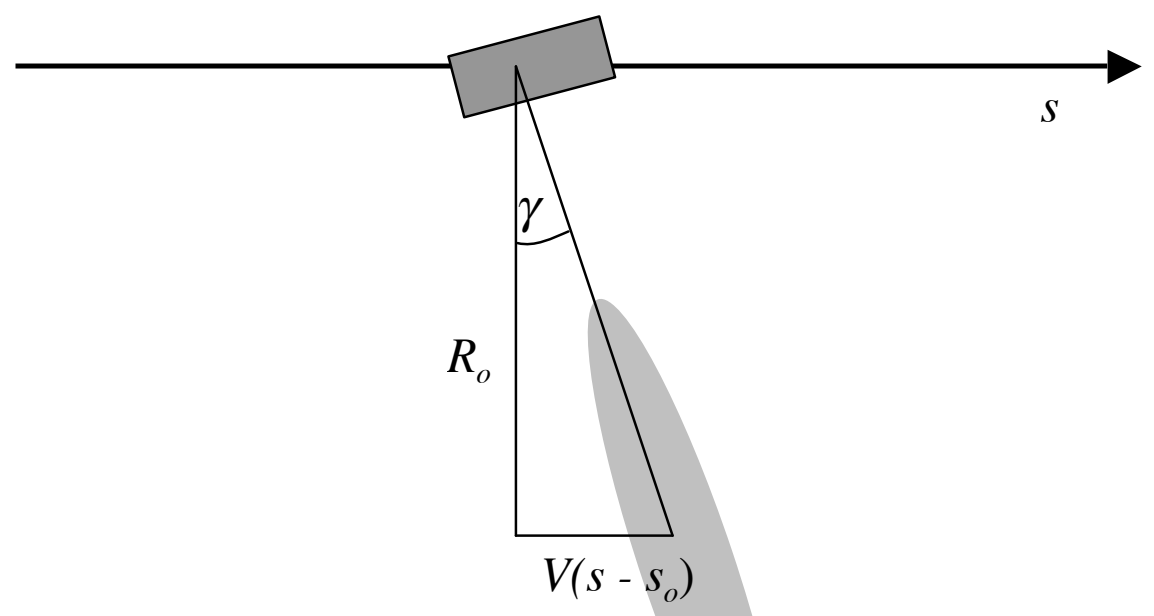

The squint angle is simply

$\gamma=\tan ^{-1}\left(\frac{V\left(s-s_{o}\right)}{R_{o}}\right)$

For the ERS, ENVISAT and ALOS satellites, the squint angle is adjusted so the zero Doppler occurs roughly in the center of the antenna beam pattern. If the satellite orbit was circular and the earth was a non-rotating sphere, then the zero Doppler would correspond to a zero squint angle.

For interferometry it is important that the beam patterns of the reference and repeat orbits have a large overlap $(>50 \%)$ at a given along-track spacecraft position. The requirement is that the Doppler centroid of the reference and repeat passes agree to about $1 / 2$ of the pulse repetition frequency $(P R F)$. In late 1999, the gyroscopes on ERS-2 failed so it became difficult to control 
the squint angle of the spacecraft. Data acquired after this date may have a Doppler centroid outside of the acceptable range and thus may be useless for interferometry. The squint angle for the ALOS satellite is well controlled and the Doppler centroid is usually less than $100 \mathrm{~Hz}$ so we commonly focus the images at zero Doppler.

\section{References}

Curlander and McDonough, Synthetic Aperture Radar: Systems \& Signal Processing, Chapter 4, John Wiley \& Sons, New York, 1991.

Madsen, S. S., Estimating the Doppler centroid of SAR data, IEEE Trans. Aerospace and Electronic Systems, AES-25, 134-140, 1989.

\section{Problems}

1) Explain why the raw signal data are provided as complex numbers. Why are the numbers restricted to the range $0-31$ ?

2) Why is the SAR processing done in patches rather than all at once? What is the minimum possible patch size?

3) Most SAR instruments emit a frequency modulated chirp rather than a short pulse. Why? Write a Matlab program to deconvolve the FM chirp for ERS and reproduce the impulse response function shown in Figure B4.

4) Make a plot of the real and imaginary parts of the function given in (B5) for a time interval of -2 to 2 seconds. Use $R_{o}=850 \mathrm{~km}, \dot{R}_{o}=0, \ddot{R}_{o}=\frac{V^{2}}{R_{o}}$ where $V=7125 \mathrm{~ms}^{-1}$.

5) Derive equation (B12)

6) Derive equation (B13). (You may need to look back at Appendix A.) 
7) Explain qualitatively how a SAR antenna should be squinted (turned) to keep the Doppler centroid of the return echoes close to zero for the following three cases: (A) an elliptical orbit about a spherical, non-rotating earth; (b) a circular orbit about an elliptical and nonrotating earth; (c) and a circular orbit about a rotating, spherical earth. 
Appendix C - InSAR Summary

\section{Forming an interferogram}

Creating an interferogram from two Single Look Complex (SLC) images involves three basic steps. The first step is to align the reference and repeat images to sub-pixel accuracy. This is done by two dimensional cross correlation (xcorr in GMTSAR) of hundreds of small sub-patches (e.g., 64 x 64) taken from the master and slave images. Then a 6-parameter affine transformation is determined using a robust 2-D fit to the offset data (i.e. trend2d in GMT). This is used to align the slave image to the master image and the actual alignment is done in the SAR processor (esarp in GMTSAR). The second step is to multiply the two SLC images to form the complex interferogram. Of course, the complex number $C(\mathbf{x})$ in each pixel of the SLC image can be written as an amplitude $A(\mathbf{x})$ and phase $\phi(\mathbf{x})$.

$C(\mathbf{x})=A(\mathbf{x}) e^{i \phi(\mathbf{x})}$

where $\mathbf{x}=(\rho, a)$ is the position vector consisting of range $\rho$ and azimuth $a$. Their product is

$C_{2} C_{1}^{*}=A_{1} A_{2} e^{i\left(\phi_{2}-\phi_{1}\right)}=R(\mathbf{x})+i I(\mathbf{x})$

The phase of the interferogram is extracted in the usual way.

$\left(\phi_{2}-\phi_{1}\right)=\tan ^{-1}\left(\frac{I}{R}\right)$

An example of the phase of reference and repeat images and their phase difference is shown in Figure C1. 

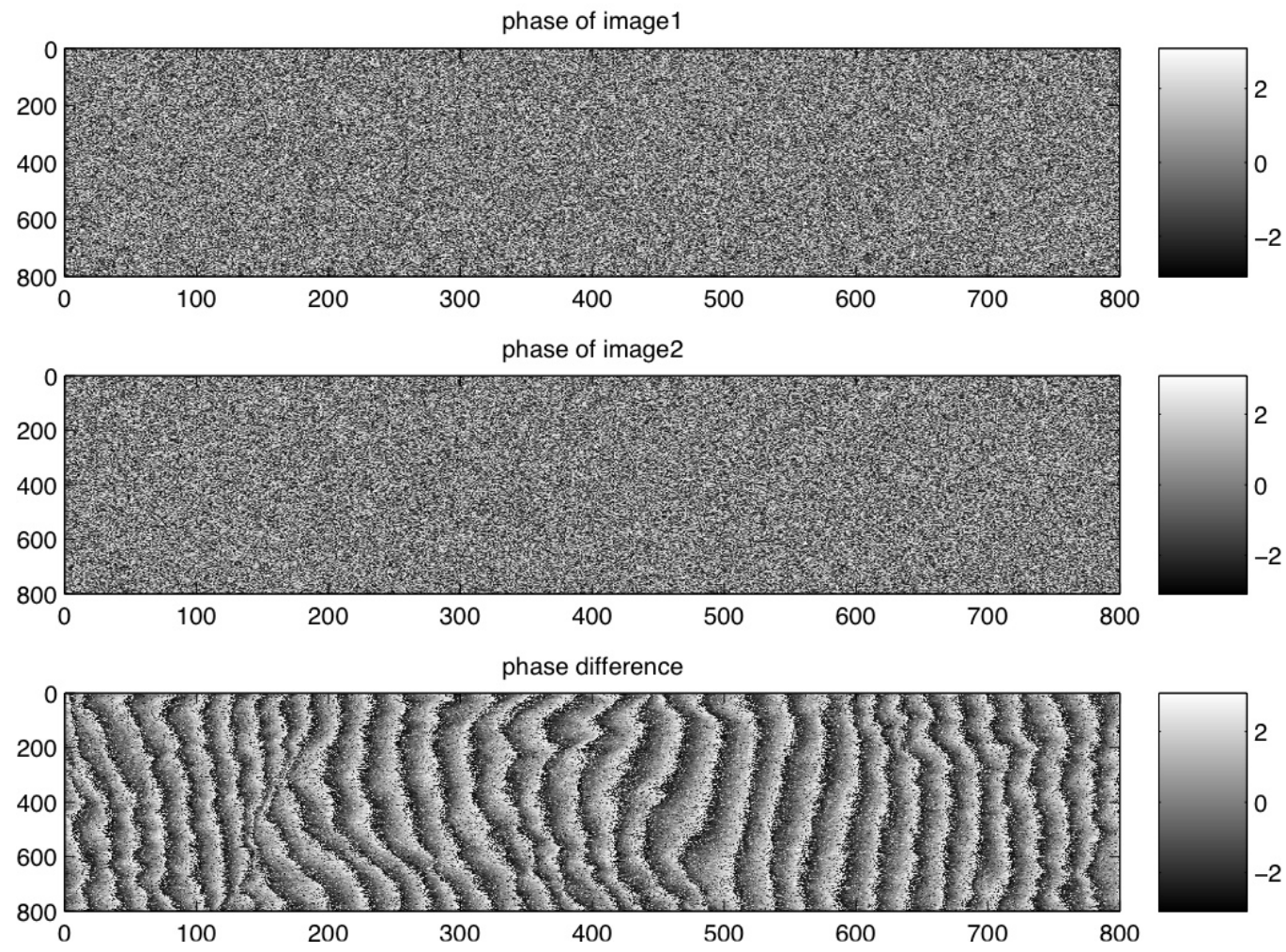

Figure C1. (upper) phase of reference SLC file. (center) phase of repeat SLC file, and (lower) phase difference of repeat - reference calculated using equations (C2) and (C3). The fringes across the interferogram are mostly due to the curvature of the earth where the fringe rate is proportional to the perpendicular baseline between the reference and repeat images.

\section{Contributions to Phase}

The phase of the interferogram has many components as described in the following equation.

phase $=$

$$
\begin{aligned}
& \text { earth curvature (almost a plane, known) + } \\
& \text { topographic phase (broad spectrum) + } \\
& \text { surface deformation (broad spectrum, unknown) + } \\
& \text { orbit error (almost a plane, largely known) + } \\
& \text { ionosphere delay (a plane or } 40-\mathrm{km} \text { wavelength waves) + } \\
& \text { troposphere delay (power law, unknown) + } \\
& \text { phase noise (white spectrum, unknown) }
\end{aligned}
$$

Normally one is trying to isolate the phase due to surface deformation from all the other contributions. The largest known contributions come from the irregular shape of the earth which can be divided into an earth curvature component and a topography component. Initially we'll separate these two components but later we'll combine them to form a complete correction for the earth's shape. In the next sections of this Appendix 
we derive approximate expressions for the phase and phase gradient due to earth curvature. This is followed by a derivation of the critical baseline, point scatterers, phase due to topography, and altitude of ambiguity. These are the standard equations and derivations presented previously [Rosen et al., 1996; Joughin et al., 1996; Sandwell and Price, 1998] and the derivations are useful for exposing concepts such as parallel and perpendicular baseline. However, this standard geometric model is no longer adequate for achieving high accuracy results, especially for L-band interferometry where long baselines are possible. In the final section of the Appendix we derive the more exact formulas that are implemented in our GMTSAR code in phasediff. Actually the more exact formulas are also conceptually and mathematically simpler than the approximate formulas and they should be used routinely.

\section{Phase due to earth curvature}

The geometry of repeat-pass interferometry is shown in Figure C2.

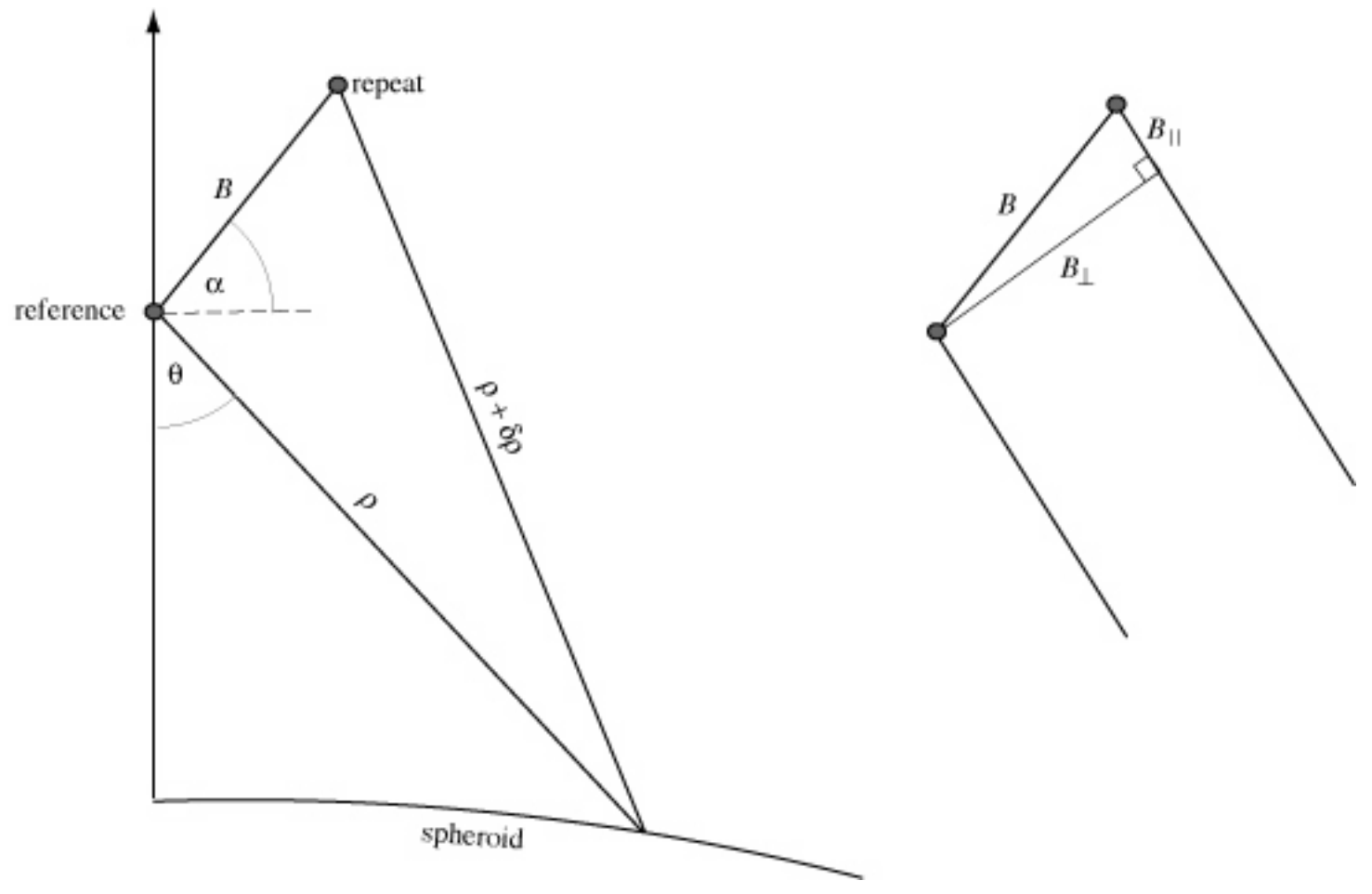

Figure C2. Geometry of repreat-pass interferometry. The important parameters are:

$\rho \quad-\quad$ range from reference track to reflector $(\sim 830 \mathrm{~km}$ for ERS)

$B \quad$ - $\quad$ total baseline distance between reference and repeat track

$\theta \quad$ - $\quad$ look angle $\left(\sim 20^{\circ}\right.$ for ERS)

$\alpha \quad$ - $\quad$ angle between the baseline vector and the tangent plane 
For discussion purposes one can divide the baseline into parallel and perpendicular components given by

$$
\begin{aligned}
& B_{\|}=B \sin (\theta-\alpha) \\
& B_{\perp}=B \cos (\theta-\alpha)
\end{aligned}
$$

Note since the look angle $\theta$ changes across the swath, the notion of a single perpendicular baseline for an interferometric combination is only approximate. The phase difference $\phi$ to a point on the ground is related to the range difference $\delta \rho$.

$$
\phi=\frac{4 \pi}{\lambda} \delta \rho
$$

where $\lambda$ is the wavelength of the radar. The law of cosines provides the relationship among the repeat-pass range, the reference-pass range, the baseline length $B$ and the baseline orientation $\alpha$.

$(\rho+\delta \rho)^{2}=\rho^{2}+B^{2}-2 \rho B \sin (\theta-\alpha)$

Next we make two standard approximations that are useful for introducing interferometric concepts but are unnecessary and also lead to inaccurate results. First we assume $\delta \rho<\rho$ so we have

$$
\delta \rho=\frac{B^{2}}{2 \rho}-B \sin (\theta-\alpha)
$$

Furthermore since $B<\rho$ the parallel ray approximation yields. 
$\phi=\frac{-4 \pi}{\lambda} B \sin (\theta-\alpha)$

The phase difference depends on the parallel component of the baseline. The derivative of the phase with respect to range is

$\frac{\partial \phi}{\partial \rho}=\frac{-4 \pi}{\lambda} B \cos (\theta-\alpha) \frac{\partial \theta}{\partial \rho}$

This phase gradient depends on two terms, the perpendicular component of the baseline $B_{\perp}=B \cos (\theta-\alpha)$ and the derivative of look angle with respect to range $\delta \theta / \delta \rho$. The perpendicular baseline varies slightly with look angle across a typical SAR image. The change in look angle usually increases with range so $\delta \theta / \delta \rho>0$. However, when the local terrain slope exceeds the look angle (actually the incidence angle), an increase in look angle does not produce a corresponding increase in range. This is the layover geometry where $\delta \theta / \delta \rho<=0$.

\section{Look angle and incidence angle for a spherical earth}

In order to calculate the derivative of look angle with respect to range $\delta \theta / \delta \rho$ we make the approximation that the earth is locally spherical although we will adjust the local radius of the Earth using the WGS84 ellipsoid (C11). While this approximation is good locally, there can be large differences between the elliptical earth model and the local radius for long SAR swaths. This difference will produce topographic fringes in longstrip interferograms depending on the interferometric baseline. We will deal with this issue later by including the ellipsoidal radius minus the local radius into a topographic correction term. The local earth radius is given by

$$
r_{e}(\varphi)=\left(\frac{\cos ^{2} \varphi}{a^{2}}+\frac{\sin ^{2} \varphi}{c^{2}}\right)^{-1 / 2}
$$


where $\varphi$ is latitude, and $a$ and $c$ are the equatorial $(6378 \mathrm{~km})$ and polar $(6357 \mathrm{~km})$ radii, respectively.

The look angle of the radar depends only on the local earth radius $r_{e}$, the range to the sphere $\rho$, and the height of the spacecraft above the center of the Earth $b$ as shown in the following diagram.

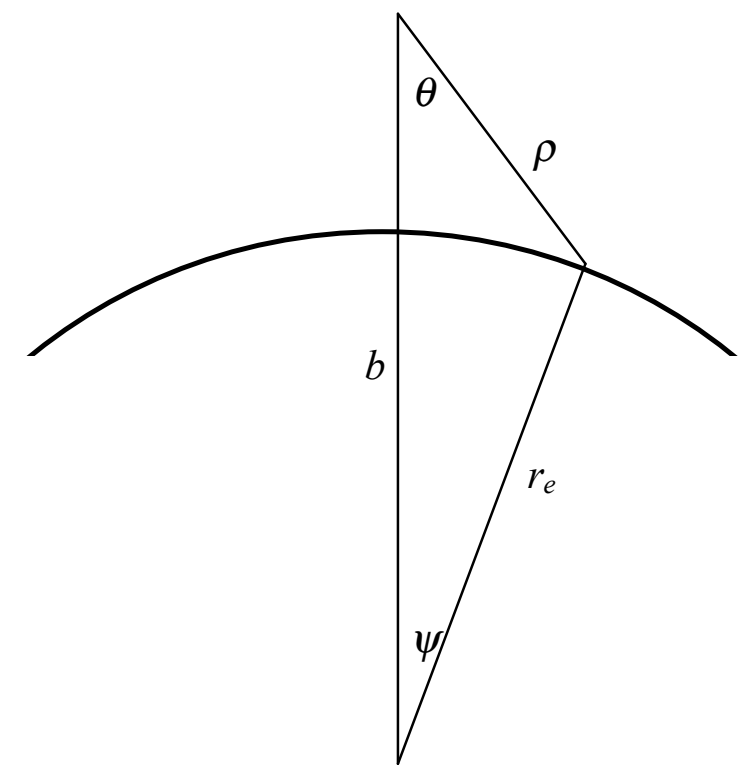

Figure C3 Triangle formed by the range $\rho$, radius of the earth $r_{e}$ and spacecraft height $b$.

Using the Law of cosines one finds

$\eta=\cos \theta=\frac{\left(b^{2}+\rho^{2}-r_{e}^{2}\right)}{2 \rho b}$

On a sphere, the incidence angle is greater than the look angle by the angle $\psi$.

To determine the derivative of the phase $(\mathrm{C} 10)$ we need to determine the derivative of the look angle with respect to range from equation (C12). After a little algebra, we find an expression for the phase gradient due to earth curvature. 


$$
\frac{\partial \phi}{\partial \rho}=\frac{-4 \pi B}{\lambda \rho} \frac{\cos (\theta-\alpha)}{\sin \theta}\left(\cos \theta-\frac{\rho}{b}\right)
$$

Using (C9), one can also derive an expression for total phase versus range.

$$
\phi=\frac{-4 \pi B}{\lambda}\left[\left(1-\eta^{2}\right)^{1 / 2} \cos \alpha-\eta \sin \alpha\right]
$$

where $\eta$ given in equation $(\mathrm{C} 12)$. Equation $(\mathrm{C} 14)$ is the approximate earth curvature correction that was used in our older codes.

\section{Critical baseline}

If the fringe rate across the interferogram due to earth curvature exceeds $2 \pi$ radians per range cell then the reference and repeat images will be completely decorrelated. Consider a single range pixel of length $\Delta \rho=C \tau / 2$. The phase of this pixel in the reference image is the vector sum of the phase from all of the scatterers in the pixel. The repeat image will have the same scatterers. However if the baseline is critical, there will be an additional $2 \pi$ phase delay across the range cell that will cause the sum of the scatterers to be randomly different from the reference image. This is called baseline decorrelation and the length at which complete decorrelation occurs is the critical baseline.

For this calculation, we start with equation (C13) and make a flat-earth approximation $\rho / b=0$. To avoid baseline decorrelation, change in phase with range must be less than

$$
\frac{\partial \phi}{\partial \rho}=\frac{-4 \pi B_{\perp}}{\lambda \rho} \frac{\cos \theta}{\sin \theta}<\frac{2 \pi}{\Delta \rho}
$$

The baseline where the fringe rate equals the critical value is 
$B_{c}=\frac{\lambda \rho}{C \tau} \tan \theta$

For the parameters of the ERS satellite the critical baseline is $1100 \mathrm{~m}$ (Tabel 1). For topographic recovery, a baseline of about $1 / 4$ critical is optimal. Of course for change detection, a zero baseline is optimal but not usually available.

Table 1. Comparison of critical baseline

\begin{tabular}{|l|c|c|c|}
\hline look angle & $23^{\circ}$ & $34^{\circ}$ & $41^{\circ}$ \\
\hline $\begin{array}{l}\text { ERS/ENVISAT } \\
16 \mathrm{MHz}\end{array}$ & $1.1 \mathrm{~km}$ & 2.0 & 2.9 \\
\hline $\begin{array}{l}\text { ALOS FBD } \\
14 \mathrm{MHz}\end{array}$ & 3.6 & 6.5 & 9.6 \\
\hline $\begin{array}{l}\text { ALOS FBS } \\
28 \mathrm{MHz}\end{array}$ & 7.3 & 13.1 & 18.6 \\
\hline
\end{tabular}

ERS/ENVISAT - altitude $=790 \mathrm{~km}$, wavelength $=56 \mathrm{~mm}$

ALOS - altitude $=700 \mathrm{~km}$, wavelength $=236 \mathrm{~mm}$

Shaded area is most common mode for interferometry.

\section{Persistent point scatterer and critical baseline}

Consider a ground range resolution of single pixel, which is related to the pulse length and the incidence angle $R_{r}=C \tau /(2 \sin \theta)$. This cell is imaged from two positions separated by a perpendicular baseline (Figure C4). If the resolution cell contains only a single persistent scatterer then the change in the viewing geometry will not change the range to the single scatterer and the pixel will remain coherent. However, if there is a uniform distribution of persistent scatterers in the cell then the scatterers at the edges of the cell will have a change in range of $\delta \rho=\frac{R_{r}}{2} \sin \delta \theta \cos \theta$. If this is less than a quarter of the wavelength then the phase shifts at the ends of the resolution cell will not destroy the correlation in the cell. Note that since the path delay is $2 \delta \rho$, the phase delay is one half 
wavelength. Working through the algebra one finds that this critical baseline is the same as found above.

$B_{c}=\frac{\lambda \rho}{2 R_{r} \cos \theta}=\frac{\lambda \rho}{C \tau} \tan \theta$

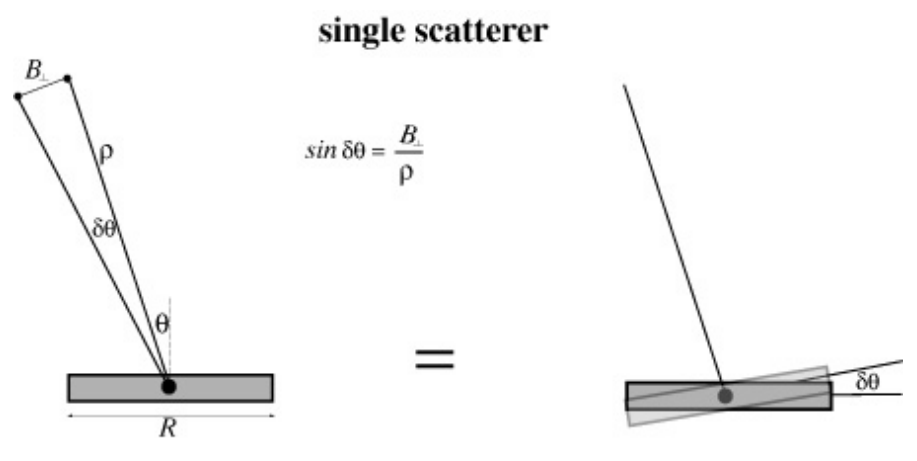

multiple scatterers

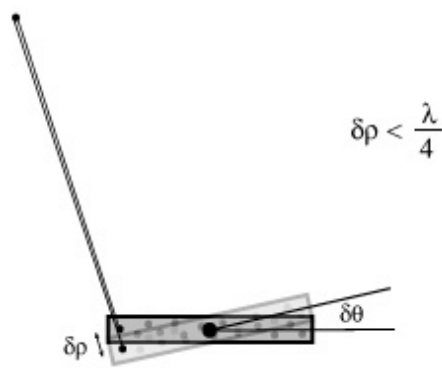

Figure C4. Diagrams showing the effects of changing viewing angle $\delta \theta$ for a resolution cell of length $R$. (upper) point scatterer has no phase change with viewing angle. (lower) has multiple scatterers where the vector sum of the phase will vary with viewing angle in a random way causing decorrelation.

\section{Phase due to topography}

One can use the approximate formula (C9) for the phase due to earth curvature to also calculate the topographic phase. The actual radius of the earth (land), $r$, is usually greater than the radius of the spheroid $r_{e}$ and this difference is geometric elevation. The phase due to the actual topography can be expanded in a Taylor Series about $r_{e}$. 
$\phi(r)=\phi\left(r_{e}\right)+\left.\frac{\partial \phi}{\partial r}\right|_{r_{e}}\left(r-r_{e}\right)+\left.\frac{1 \partial^{2} \phi}{2 \partial r^{2}}\right|_{r_{e}}\left(r-r_{e}\right)^{2}+\cdots$

Using equations $\mathrm{C} 9$ and $\mathrm{C} 12$ one can calculate the first two derivatives. It turns out that the second derivative is about $\left(r-r_{e}\right) / r$ times the first derivative (i.e., $2.7 / 6371$ for our area) so we only need to keep the first two terms in the series. The first term is $\mathrm{C} 12$ while the second term is

$$
\frac{\partial \phi}{\partial r}\left(r_{e}\right)=\frac{-4 \pi r_{e}}{\lambda \rho b} \frac{B \cos \left(\theta_{e}-\alpha\right)}{\sin \theta_{e}}
$$

where $\theta_{e}$ is the look angle to the spheroid $(\mathrm{C} 12)$. The mapping of total unwrapped phase into elevation as a function of range is

$$
\left(r-r_{e}\right)=\frac{-\lambda \rho b}{4 \pi r_{e}} \frac{\sin \theta_{e}}{B \cos \left(\theta_{e}-\alpha\right)}\left(\phi-\phi_{e}\right)
$$

One should remember that the unwrapped interferogram does not provide the complete phase difference $\phi-\phi_{\mathrm{e}}$ since there is an unknown constant of integration. While this formula is useful for demonstrating that the magnitude of the topographic phase correction is proportional to the perpendicular baseline, the formula is only approximate. The more exact approach, discussed next, is used in the GMTSAR code.

\section{Altitude of ambiguity}

The fringe rate due to topography can be reformulated to provide the error in the elevation model that will produce one fringe $(2 \pi)$ error in the interferogram. Again we'll use a flat-earth geometry so $b / r_{e}=1$. From equation C20 we find the altitude of ambiguity is 
$h_{a}=\frac{\lambda \rho \sin \theta_{e}}{2 B_{\perp}}$

For the case of ERS with a perpendicular baseline of $100 \mathrm{~m}$, this altitude of ambiguity is about $90 \mathrm{~m}$. For change detection, a higher number is better.

Phase due to earth curvature and topography - exact formula

The above text describes the standard approximations that are in most InSAR packages such as ROI_PAC and Gamma. However there is a second order term in the derivation that can become important when the baseline is long. We start the derivation with equation (C7) where we have expanded the left side and cancelled the $\rho^{2}$ terms.

$$
2 \rho \delta \rho+\delta \rho^{2}=B^{2}-2 \rho B \sin (\theta-\alpha)
$$

This can be rewritten as

$$
\delta \rho=-B \sin (\theta-\alpha)+\frac{1}{2 \rho}\left(B^{2}-\delta \rho^{2}\right)
$$

Now in the approximate derivation in the sections above we found a good approximation to the range change is given by $\delta \rho=-B \sin (\theta-\alpha)$. If we use this as a guess for the right hand side of equation $(\mathrm{C} 23)$ we find a second order approximation to the range change is

$$
\delta \rho=-B \sin (\theta-\alpha)+\frac{B^{2}}{2 \rho}\left[1-\sin ^{2}(\theta-\alpha)\right]
$$

or

$\delta \rho=-B \sin (\theta-\alpha)+\frac{B^{2}}{2 \rho} \cos ^{2}(\theta-\alpha)$

Note that we could iterate on this substitution to come up with a more accurate correction. The error introduced by neglecting the second order term is proportional to 
the perpendicular baseline squared and it is inversely proportional to range. Next we estimate the magnitude of the second order term in relation to the first order term

$$
\text { ratio }=\frac{B \cos ^{2}(\theta-\alpha)}{2 \rho \sin (\theta-\alpha)}
$$

Note that this ratio is wavelength independent. Setting $\alpha=0$ and using a nominal look angle of $30^{\circ}$, the ratio is

$$
\text { ratio }=\frac{3 B}{4 \rho}
$$

For a nominal range of $750 \mathrm{~km}$ and a baseline of $100 \mathrm{~m}$ the ratio is $10^{-4}$ and for a baseline of $2000 \mathrm{~m}$, which is typical for an L-band interferogram, the ratio is $2 \times 10^{-3}$. We show below that this corresponds to a $0.6 \mathrm{~m}$ phase ramp across an image so this second order term should not be neglected. Moreover the phase ramp from the second order term always has the same sign even when the sign of the perpendicular baseline changes. Therefore software that performs baseline re-estimation in an effort to reduce phase ramps will have a biased baseline estimate that will consistently amplify the topographic phase correction. For a large stack of interferograms, this could appear as a systematic elevation-dependent error.

The phase correction due to earth shape depends on the look angle as a function of range. In the main section of this document we discussed an algorithm for mapping a topographic grid from longitude, latitude, and topography above the ellipsoid to range, azimuth, and topography $t(\rho, a)$ above a local spherical shape for the earth. Using this information we can combine the earth curvature correction and the topographic phase correction into a single correction. The algorithm is:

1) Read a row of data from the reference and repeat SLC files so every pixel in the reference image can be assigned a range $\rho$ and azimuth $a$. 
2) Using the precise orbital information compute the radius of the spacecraft orbit for the reference image $b(a)$, the length of the baseline $B(a)$, and the orientation of the baseline $\alpha(a)$.

3) Interpolate the topography to each range pixel and compute the look angle using the following formula.

$$
\theta=\cos ^{-1}\left\{\frac{\left(b^{2}+\rho^{2}-\left(r_{e}+t(\rho, a)\right)^{2}\right)}{2 \rho b}\right\}
$$

4) Now that we have the look angle for each range pixel, we can calculate the phase correction to be applied to the repeat image.

$$
\phi=-\frac{4 \pi B}{\lambda} \sin (\theta-\alpha)+\frac{2 \pi B^{2}}{\lambda \rho} \cos ^{2}(\theta-\alpha)
$$

5) Multiply the repeat image by the complex phase and form the interferogram. Note this phase correction should be applied before any multilook averaging or filtering.

$$
R(\mathbf{x})+i I(\mathbf{x})=C_{2} C_{1}^{*} e^{i \phi}
$$

6) Extract the interferometric phase that is now corrected for both the earth curvature and the topographic phase.

$$
\left(\phi_{2}-\phi_{1}\right)=\tan ^{-1}\left(\frac{I}{R}\right)
$$

An example of a $1.95 \mathrm{~km}$ long baseline interferogram that was constructed both with and without the second order phase correction is shown in Figure C5. The interferogram with the approximate phase correction has a ramp across the image as well a small phase 
variation due to topography. Note there is $3200 \mathrm{~m}$ of relief within this interferogram and for this baseline the altitude of ambiguity is $27.4 \mathrm{~m}$ so there are 120 fringes in the full interferogram (Figure C5 (a)) that need to be removed. The residual fringes (b) could be residual topographic phase but also could be tropospheric delay or orbital error.

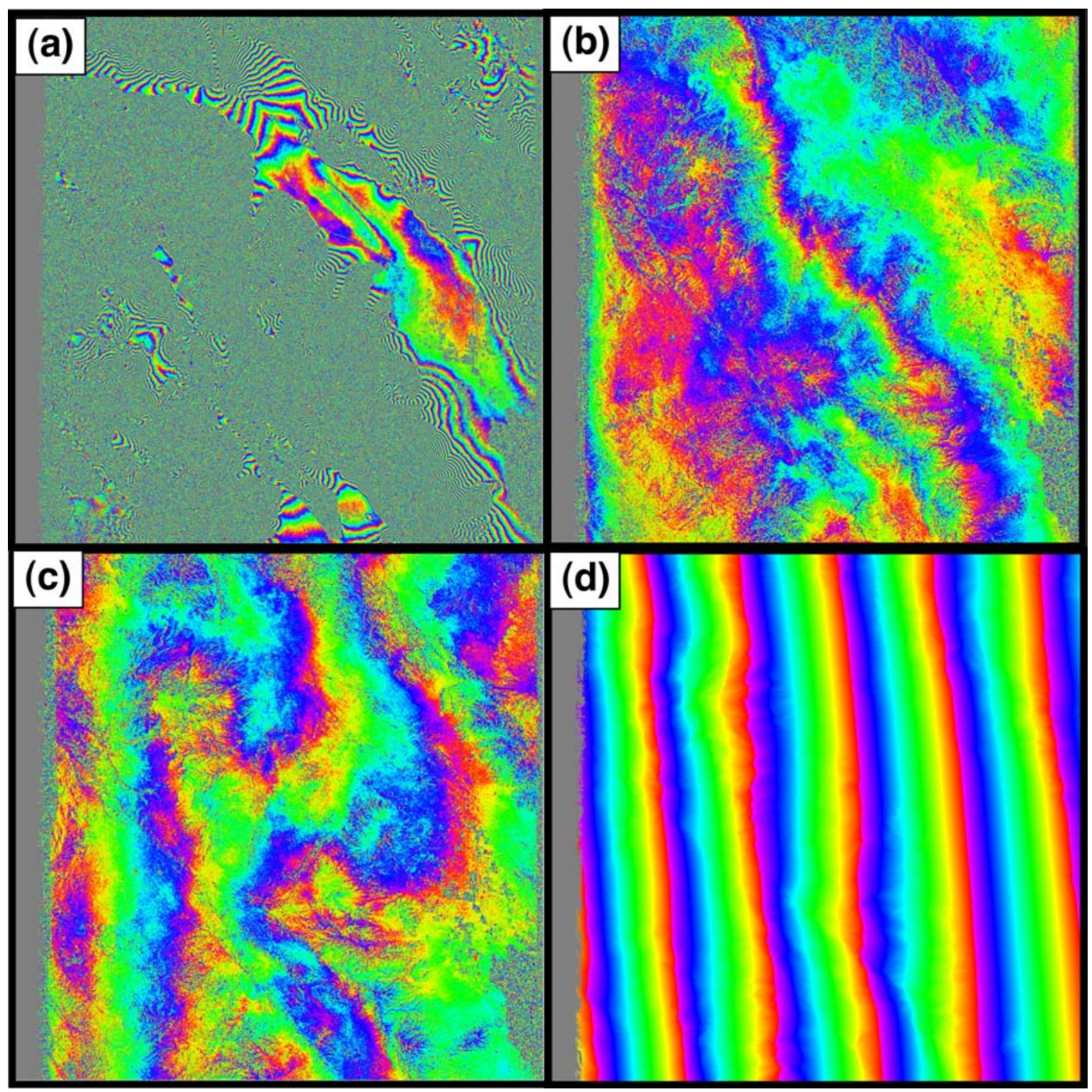

Figure C5. (a) A $1.95 \mathrm{~km}$ baseline interferogram with no topography correction has 120 fringes that need to be accurately removed. (b) Same interferogram corrected using the exact formulas (C26) and (C27). (c) Same interferogram corrected using the approximate formulas (C12) and (C14). (d) difference between exact and approximate corrections corresponds to a $0.6 \mathrm{~m}$ ramp. The interferogram is marked as the blue baseline shown in Figure 6 (T213, F0660, O 02220 to 02891) 


\section{References}

Joughin, I., M. Winebrenner, R. Fahnestock, R. Kwok, and W. Krabill (1996), Measurement of ice sheet topography using satellite-radar interferometry, J. Glaciology, 42, 10-22.

Rosen, P. A., S. Hensley, H. A. Zebker, F. H. Webb, and E. Fielding (1996), Surface deformation and coherence measurements of Kilauea Volcano, Hawaii from SIR-C radar interferometry, J. Geophys. Res., 101(E10), 23109-23125.

Sandwell, D. T., and E. J. Price (1998), Phase gradient approach to stacking interferograms, $J$. Geophys. Res., 103(B12), 30183-30204.

\section{Problems}

1) Develop a formula similar to (C12) for the incidence angle as a function of local earth radius $r_{e}$, the range to the sphere $\rho$, and the height of the spacecraft above the center of the Earth $b$.

2) Make an interferogram from two SLC images using Matlab. Online you will find 2 binary SAR image files, *.SLC, and 2 ascii header files, *.PRM (download at ftp://topex.ucsd.edu/pub/class/rs/LAB8 ). The ftp site also contains some matlab code to read the data and get started (lab8start.m). This is an ERS-1 to ERS-2 tandem pair from the Salton Sea, perpendicular baseline $58 \mathrm{~m}$. The *.PRM files have been included for your information only and will not be of much use for the problems below. This lab illustrates SAR interferometry. First look at the phase of the reference image. It will look like random noise. Then look at the phase of the repeat image. It will also look like noise. Finally examine the phase difference. This will show interferometric fringes across the image related to the curvature of the Earth.

a) Make the best-looking amplitude image that you can using images 1 and 2. This could involve low-pass filtering and perhaps averaging the two images. I can't provide a recipe for doing this but note that the pixels are $16 \mathrm{~m}$ in the range direction and only 4 $\mathrm{m}$ in the azimuth direction (top-to-bottom) so the filter should be taller than it is wide by about 4 times. Remember these are complex numbers so would one construct 
amplitude then filter or filter the real and imaginary parts and then make amplitude? When and how should the images be averaged?

b) Make the interferogram and filter it to make the best-looking phase map. Should one filter before or after interferogram formation? Should one filter the real and imaginary components or filter the phase?

c) Do the best that you can to remove the phase ramp across the image. How do you estimate the phase ramp and what is the best way to remove it?

d) Try to unwrap the phase using Matlab?

3) Derive equation (C13). What happens when the change in phase with increasing range exceeds $2 \pi$ radians per range resolution cell?

4) Give two reasons why the critical baseline for ALOS is more than 10 times longer than for ERS.

5) What happens to the phase of an interferogram in areas where the slope of the topography facing the radar exceeds the incidence angle of the radar?

6) Derive equation (C26). What is the correct zero level for the topography in this equation? 
Appendix D ScanSAR processing and interferometry [Tong et al., 2010]

The systematic observation strategy of ALOS PALSAR provides strip-mode SAR imagery along every ascending orbital track and ScanSAR imagery along every third descending orbital track. Therefore to obtain a second look direction of PALSAR interferometry sometimes requires processing ScanSAR to ScanSAR mode interferograms. The method is based on the proposal by Bamler and Eineder [1996] that, with proper pre-processing, standard strip-mode software can be used to construct phasepreserving SAR images and ultimately interferograms. The main advantage of using the standard strip-mode approach, instead of the traditional SPECAN approach [Sack et al., 1985], is that existing and well tested, strip-mode InSAR processing software can be used. The main disadvantage of this approach is that the zero-padding between the bursts wastes considerable disk space and computer time. Nevertheless the two approaches should provide equivalent results.

Our strip-mode preprocessor for ALOS was developed and tested using data along a descending orbital track (T538) over Southern California. This track contains two permanently installed radar corner reflectors that are used by JAXA to provide radiometric and geometric calibration for the lifetime of the mission. JAXA has collect PALSAR data over these reflectors in a variety of modes on both ascending and descending tracks. We have extended the ALOS preprocessing software to first perform ScanSAR to FBD mode interferometry and second to perform the more challenging ScanSAR to ScanSAR interferometry where a significant burst overlap is needed. Indeed both luck and accuracy are required to achieve a full swath-width ScanSAR to ScanSAR interferogram. The ScanSAR acquisition geometry and parameters for PALSAR are provided in Tables D1 and D2. The 5 subswaths cover an area $\sim 350 \mathrm{~km}$ wide. Following Balmer and Einder [1996] the ScanSAR data are zero-padded to construct swath type data. The original WB1 file contains the bursts of all 5 sub-swaths as consecutive rows. The preprocessor separates the data into 5 separate files where missing lines between the bursts are filled with zeros. Zeros are also added to the end of each echo to match the length of a standard FBD data file. In addition, the first 12 lines of each burst are zero-padded because these data are incorrect [Masanobu Shimada, 
personal communication, 2008]. Since the sub-swaths are processed independently and their amplitude and interferometric phase are recombined in the latitude-longitude coordinate system, an accurate geometric model, precise orbit, and consistent set of processing parameters is required to achieve a seamless recombination. The preprocessing code is rather complex because the pulse repetition frequency (PRF) of the PALSAR echoes varies in three ways. First as shown in Table D2, each sub-swath has its own PRF optimized to reduce crosstalk. Second, the PRF can change along the satellite track on any one of the sub-swaths. A PRF change on any sub-swath changes the time interval and number of zero-pad lines needed on all the other sub swaths. Finally, when considering reference and repeat images for interferometry, the PRF's on a matching subswaths can be different causing the burst alignment to change along the swath as we find in the example below. In addition each sub-swath has its own near range which, varies along the track so rows must be shifted to align the near range to the common prescribed value. Any small error in the preprocessing could result in a poorly focused image and/or low interferometric coherence.

Table D1 Pattern of bursts for the 5 sub swaths of PALSAR in WB1 mode. Sub swath 4 corresponds to the nominal FBD and FBS strip-mode imagery.

\begin{tabular}{|c|c|c|c|c|}
\hline SW1 & SW2 & SW3 & SW4 & SW5 \\
\hline & & & & \\
\hline & & & & \\
\hline & & & & \\
\hline & & & & \\
\hline & & & & \\
\hline & & & & \\
\hline & & & & \\
\hline & & & & \\
\hline
\end{tabular}


Table D2 Nominal radar parameters for each sub swath. The number of echoes in a burst, nburst, is the only fixed parameter.

\begin{tabular}{|c|c|c|c|c|c|}
\hline & SW1 & SW2 & SW3 & SW4 & SW5 \\
\hline near range (m) & 730097 & 770120 & 806544 & 848515 & 878195 \\
\hline PRF $(\mathrm{Hz})$ & 1692 & 2370 & 1715 & 2160 & 1916 \\
\hline nburst & 247 & 356 & 274 & 355 & 327 \\
\hline$\Delta t(\mathrm{~s})$ & 0.146 & 0.150 & 0.160 & 0.164 & 0.171 \\
\hline nsamples & 4976 & 4720 & 5376 & 4432 & 4688 \\
\hline off nadir (deg) & 20.1 & 26.1 & 30.6 & 34.1 & 36.5 \\
\hline
\end{tabular}

The first step in the software development was to focus the raw sub-swath data to form seamless amplitude imagery. We found that the best image quality (i.e. minimal scalloping) was achieved by setting the length of the synthetic aperture to be exactly 6 bursts. This corresponds to setting the time $(\sim 4.7 \mathrm{~s})$ and along-track distance of synthetic aperture to be exactly the same for each sub swath. We have found that by processing the SAR image to zero Doppler, the position of the radar corner reflectors in the image matches the position predicted from the orbit (zero range rate) to within 1 pixel in range and 4 pixels in azimuth. This good match provided confidence that the code is geometrically accurate.

The second step in the software development was to construct ScanSAR to swath mode interferometry. This combination should always provide interference fringes because there is $100 \%$ overlap between the sparse bursts of the ScanSAR and the complete coverage of the swath [Ortiz and Zebker, 2007]. The ALOS track 538 over Los Angeles has two FBD acquisitions and 3 ScanSAR WB1 acquisitions. The swath of the FBD data (34.3 incidence angle) overlaps with sub-swath 4 of the WB1 data. We experimented with three interferometric mode combinations, FBD to FBD, FBD to ScanSAR, and ScanSAR to ScanSAR. The FBD to FBD interferogram had a $10 \mathrm{~m}$ baseline and 46 day timespan resulting in excellent overall coherence of 0.67 . The FBD to ScanSAR interferogram had a somewhat longer baseline $121 \mathrm{~m}$ and 181 day timespan resulting in a lower average coherence of 0.38 .

The third step in the software development was to construct ScanSAR to ScanSAR interferometry. The major issue here is that there is only a $20 \%$ chance of having alongtrack alignment of the bursts between reference and repeat orbits. There is a question of how much overlap of the bursts is needed to obtain interpretable interference fringes. We 
were very fortunate that two of the ScanSAR images along track 538 have significant burst overlap (up to $78 \%$ ) and a moderate baseline of $450 \mathrm{~m}$. An interesting aspect of this interferometric pair is that they have different PRF. This produces a gradual change in burst alignment along the track from $78 \%$ at the start of the acquisition to $0 \%$ overlap after 18 bursts. The phase and coherence of sub-swath 4 of this interferogram are shown in Figure D1. Phase recovery and coherence are best at the top of the swath and gradually worsen toward the bottom. A plot of row-averaged coherence versus burst overlap fraction (Figure D1c) illustrates that a maximum coherence of about 0.35 occurs where the burst overlap is greater than $50 \%$. The coherences diminishes to below 0.2 at a burst overlap of $18 \%$. The results show that good interferometric results can be achieved when the burst overlap is greater than about $50 \%$.

Assuming there is no control on the burst alignment of reference and repeat images there is a 1 in 5 chance of this occurring from any two ScanSAR images. We used probability theory to show that 5 repeat SAR images are needed to have a $90 \%$ chance of half burst overlap for at least one pair (Table D3). One successful alignment with half burst is called an event, of which the probability is $20 \%$. We know these events are independent but not disjoint with each other. The probability for multiple SAR images can be generalized with calculation on probability of the union.

Table D3 The probability analysis on getting half burst alignment.

\begin{tabular}{|c|c|c|}
\hline Number of SAR images & $\begin{array}{c}\text { Number of interferometry } \\
\text { pairs }\end{array}$ & $\begin{array}{c}\text { Probability of getting at } \\
\text { least one pair with half } \\
\text { burst alignment }\end{array}$ \\
\hline 2 & 1 & 0.2 \\
\hline 3 & 3 & 0.488 \\
\hline 4 & 6 & 0.7379 \\
\hline 5 & 10 & 0.8926 \\
\hline 6 & 15 & 0.9648 \\
\hline 7 & 21 & 0.9908 \\
\hline 8 & 28 & 0.9981 \\
\hline
\end{tabular}



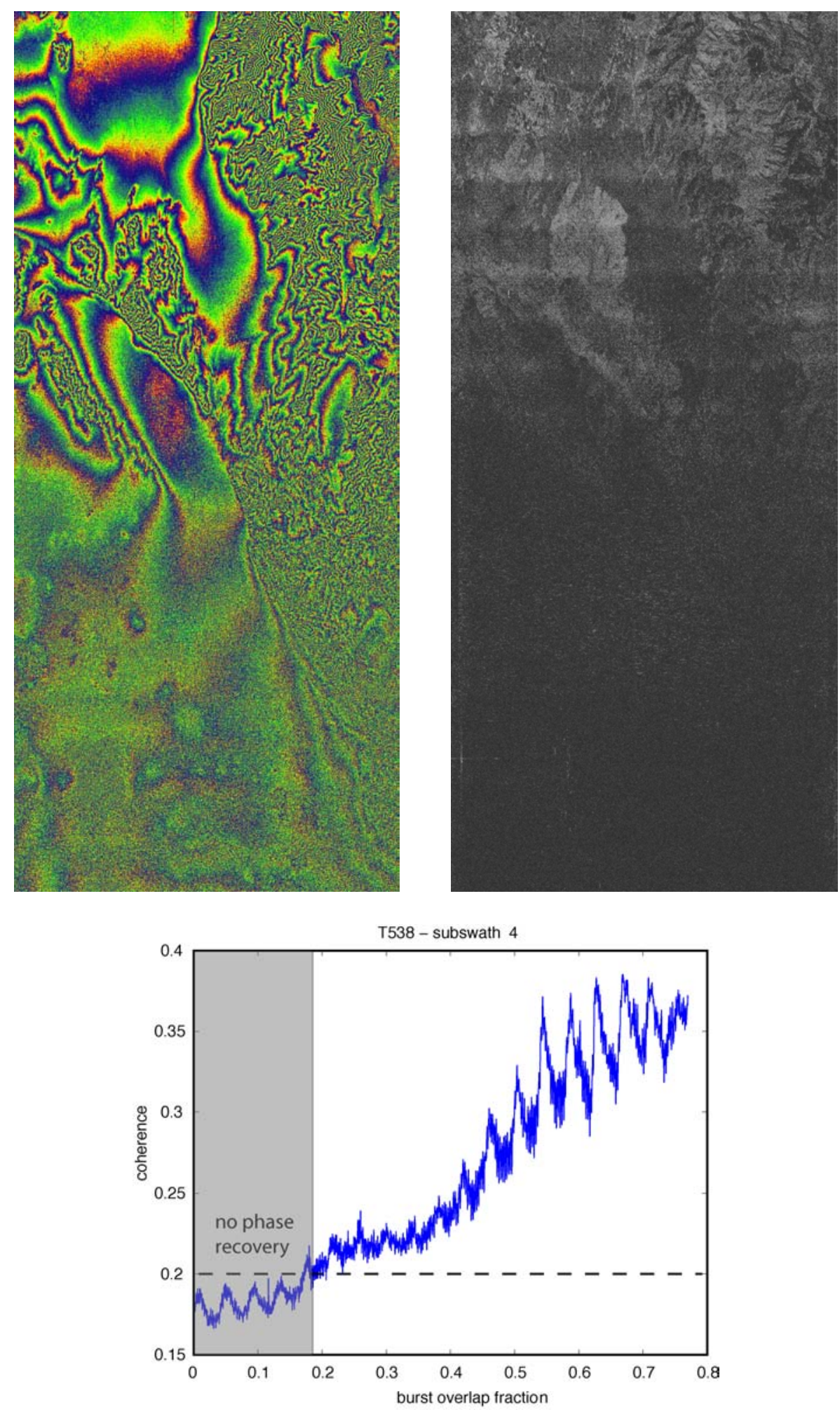

Figure D1 ScanSAR to ScanSAR interferogram for subswath 4 of track 538 across the Los Angeles basin. The perpendicular baseline is $450 \mathrm{~m}$ and the time interval is 92 days. No topographic phase has been removed. (a) interferometric phase is high at top of swath where burst overlap is large and lower toward the bottom. (b) coherence also decreases from top to bottom as burst overlap decreases. (c) row-averaged coherence versus burst overlap. 


\section{References}

Bamler, R., and M. Eineder (1996), ScanSAR processing using standard high precision SAR algorithms, IEEE Trans. Geosci. Remote Sens., 34(1), 212-218, doi:10.1109/36.481905.

Bertran-Ortiz, A., and H.A. Zebker (2007), ScanSAR-to-Stripmap Mode Interferometry Processing Using ENVISAT/ASAR Data, IEEE Trans. Geosci. Rem. Sensing, Vol. 45, No. 11, pp. 3468-3480.

Sack, M., and I. G. Cumming (1985), Application of efficient linear fm matched filtering algorithms to synthetic aperture radar processing, IEEE Proc. Part F Radar Signal Processing, 132(1), 45-57.

Tong, X., D. T. Sandwell, and Y. Fialko (2010), Coseismic slip model of the 2008 Wenchuan earthquake derived from joint inversion of interferometric synthetic aperture radar, GPS, and field data, J. Geophys. Res., 115, B04314, doi:10.1029/2009JB006625.

\section{Problems}

1) ScanSAR is a technique to construct a very wide swath radar image by emitting a burst of $\sim 300$ radar pulses on a narrow subswath and systematically cycling the near range of each burst to illuminate 5 parallel subswaths ultimately forming a wide swath. Why is it not possible to simply illuminate the wide swath with continuous wide pulses? Hint, see equation (A12).

2) How can a standard strip-mode SAR processor be used to focus ScanSAR data? What are the advantages and disadvantages of this approach?

3) Phase coherence for regular swath-mode interferometry in only possible when the baseline between the reference and repeat images is less than the critical baseline and there is significant overlap in the Doppler spectra of the reference and repeat images. What additional attribute is needed to achieve phase coherence for ScanSAR interferometry?

4) How many different interferometric combinations are possible for 10 SAR images? for 100 SAR images? for $N$ SAR images? 
Appendix E Geolocation accuracy for Pinon corner reflectors

Precise orbital information can be used to map every point on the surface of the earth (lon, lat, topography) into the range and azimuth coordinates of the radar. If the SAR image is focused at zero Doppler then the range coordinate of the mapping is simply the minimum range and the azimuth coordinate is the time of the minimum range. If the radar image was focused at a Doppler centroid other than zero, then equations 4 and 5 of the main document are the two corrections needed to transform from geographic to radar coordinates when the image is focussed at a Doppler other than zero.

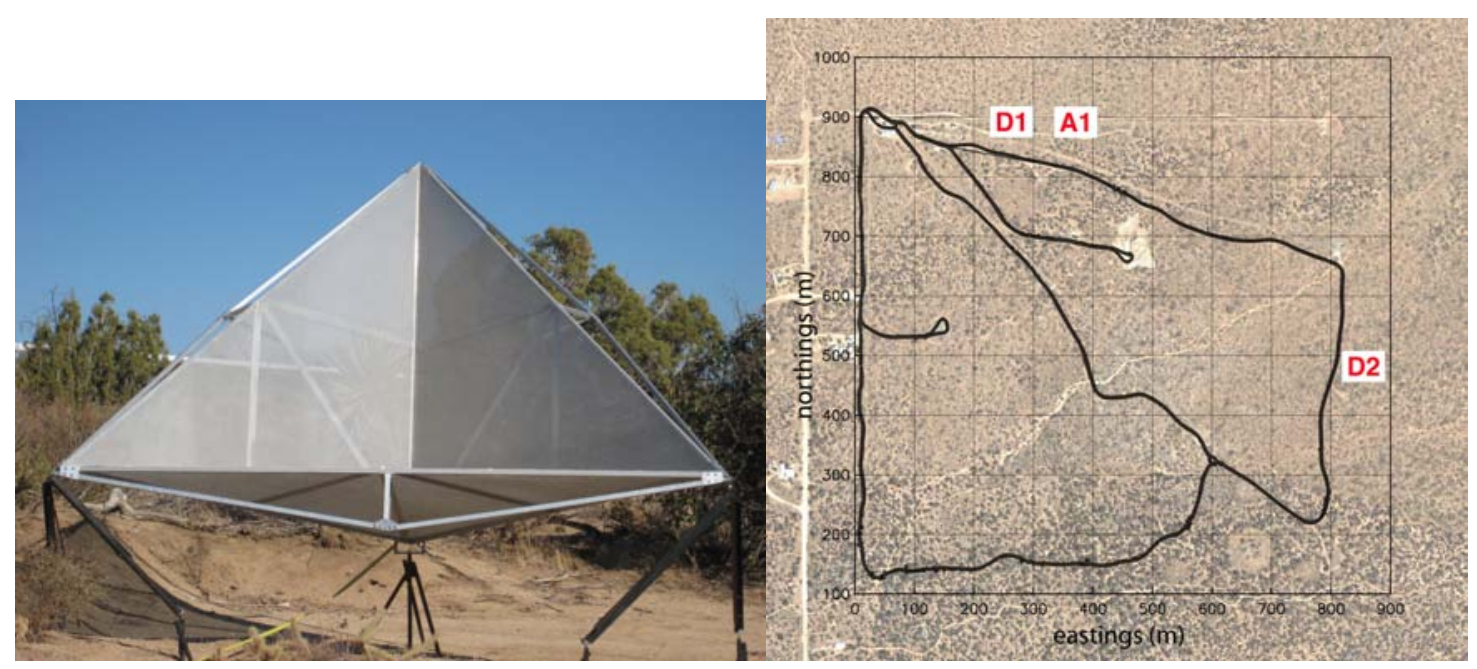

Figure E1. (left) one of the $2.4 \mathrm{~m}$ radar corner reflectors at Pinon Flat Observatory installed in 1998. (right) Satellite image of the Pinon Flat Observatory. This arid region at an elevation of $1200 \mathrm{~m}$ is relatively flat with a surface of decomposed granite sparsely covered by bush and grass. Three radar corner reflectors are oriented to reflect energy from ascending (A1 - ALOS) and descending (D1 and D2 - ERS2 and ENVISAT) passes.

Table E1. Coordinates of Radar Reflectors

\begin{tabular}{|l|l|l|l|l|l|}
\hline & \multicolumn{3}{|l|}{ position } & orientation \\
\hline & lat & lon & height & elevation & azimuth \\
\hline A1 & 33.612246 & -116.456768 & 1258.990 & $39^{\circ}$ & $257.5^{\circ}$ \\
\hline D1 & 33.612253 & -116.457893 & 1257.544 & $39^{\circ}$ & $102.5^{\circ}$ \\
\hline D2 & 33.607373 & -116.451836 & 1254.537 & $39^{\circ}$ & $102.5^{\circ}$ \\
\hline
\end{tabular}

Latitude and longitude in decimal degrees and elevation in meters relative to the WGS-84 coordinate system and ellipsoid. The survey point is the apex (lowest corner) of each reflector.

We tested the combined accuracy of the geometric model, the satellite orbits, and the range and Dopper measurements, using 3 radar corner reflectors installed at Pinon Flat 
Observatory (Figure E1 and Table E1). The positions of the corner reflectors are accurately measured using GPS. The orbits for ERS2, ENVISAT, and ALOS are determined to an accuracy of better than $30 \mathrm{~cm}$ and perhaps as good as $5 \mathrm{~cm}$. The near range and timing of the satellite are provided with the data and should have sub-meter accuracy. Using this information one can predict the range and azimuth pixel where the bright radar reflection should appear in the image. We then focus each SAR image and locate the position of the radar reflectors as bright spots. This is done by eye to an accuracy of about 1 pixel in range and about 2 pixels in azimuth. The difference between the image-based coordinate and the orbital-based coordinate provides an estimate of the overall accuracy of the system.

We performed this analysis on all ERS2 data collected after the radar reflectors were installed having Doppler centroid less than 1/2 the PRF (36 points), as well as all available ENVISAT (10 points), and ALOS data (27 points). The results for ERS2, ENVISAT, and ALOS are provided in Tables E2, E3, and E4, respectively. In the case of ERS2, the mean difference and rms deviation in range is -1.8 and 0.3 pixels respectively while the mean and rms differences in azimuth are 3.7 and 2.6 pixels respectively. The mean differences are statistically significant and this bias should eventually be included in the orbital software. ENVISAT has much larger mean differences in range and azimuth of 8.4 and 4, respectively but the rms differences are small ( 0.2 and 2.2 respectively) so these biases have been included in the software. Finally the ALOS mean differences are relatively small in both range $(-1.2+/-0.6)$ and azimuth $(0.6+/-1.1)$ so no biases have been included in the software. Overall we find that after correcting for a range bias in ENVISAT, the range and azimuth coordinates based only on the orbital information are accurate to 1 pixel in range and 2 pixels in azimuth consistent with the uncertainties in picking the bright locations in the images. Based on this analysis we conclude that ground control is not needed for InSAR processing and orbital-based image alignment should be accurate to better than about 15 $\mathrm{m}$ in range and $10 \mathrm{~m}$ in azimuth. 
Table E2. Comparison between ERS2 image and orbit for Pinon corner reflectors D1 and D2 (right).

\begin{tabular}{|l|r|r|r|}
\hline NAME & Doppler & Ri-Ro & Ai-Ao \\
\hline e2_22025_2925 & 58.3 & -1.7 & 3.0 \\
\hline e2_22025_2925 & 58.3 & -1.9 & 2.9 \\
\hline e2_22526_2925 & 81.2 & -1.5 & 5.2 \\
\hline e2_22526_2925 & 81.2 & -2.3 & 5.1 \\
\hline e2_23027_2925 & 17.1 & -0.9 & 7.2 \\
\hline e2_23027_2925 & 17.1 & -2.0 & 7.2 \\
\hline e2_23528_2925 & 78.3 & -1.7 & -0.1 \\
\hline e2_23528_2925 & 78.3 & -1.9 & -0.1 \\
\hline e2_24029_2925 & 75.4 & -2.2 & 7.6 \\
\hline e2_24029_2925 & 75.4 & -1.9 & 7.6 \\
\hline e2_24530_2925 & 141.4 & -1.8 & 2.6 \\
\hline e2_24530_2925 & 141.4 & -1.6 & 2.6 \\
\hline e2_25532_2925 & 575.5 & -1.5 & 6.1 \\
\hline e2_25532_2925 & 575.5 & -1.8 & 6.0 \\
\hline e2_26033_2925 & 42.4 & -2.1 & 3.3 \\
\hline e2_26033_2925 & 42.4 & -1.9 & 4.8 \\
\hline e2_27035_2925 & -286.5 & -1.9 & 3.5 \\
\hline e2_27035_2925 & -286.5 & -1.8 & 3.6 \\
\hline e2_27536_2925 & -295.8 & -1.9 & 7.1 \\
\hline e2_27536_2925 & -295.8 & -2.0 & 7.2 \\
\hline e2_28037_2925 & -75.7 & -1.7 & 4.3 \\
\hline e2_28037_2925 & -75.7 & -1.5 & 4.3 \\
\hline e2_28538_2925 & -1.7 & -1.7 & 2.2 \\
\hline e2_28538_2925 & -1.7 & -2.0 & 2.2 \\
\hline e2_29039_2925 & -210.2 & -1.8 & 3.8 \\
\hline e2_29039_2925 & -210.2 & -1.6 & 3.3 \\
\hline e2_33047_2925 & -625.3 & -2.1 & -3.6 \\
\hline e2_33047_2925 & -625.3 & -1.8 & -3.5 \\
\hline e2_55592_2925 & 530.8 & -1.9 & 3.6 \\
\hline e2_55592_2925 & 530.8 & -1.9 & 3.5 \\
\hline e2_58598_2925 & -203.8 & -2.3 & 2.4 \\
\hline e2_58598_2925 & -203.8 & -2.0 & 1.9 \\
\hline e2_59099_2925 & -95.8 & -1.2 & 3.3 \\
\hline e2_59099_2925 & -95.8 & -2.0 & 3.3 \\
\hline e2_61103_2925 & -83.9 & -1.7 & 4.2 \\
\hline e2_61103_2925 & -83.9 & -1.4 & 4.2 \\
\hline mean & & -1.8 & 3.7 \\
\hline rms & & 0.3 & 2.6 \\
\hline
\end{tabular}

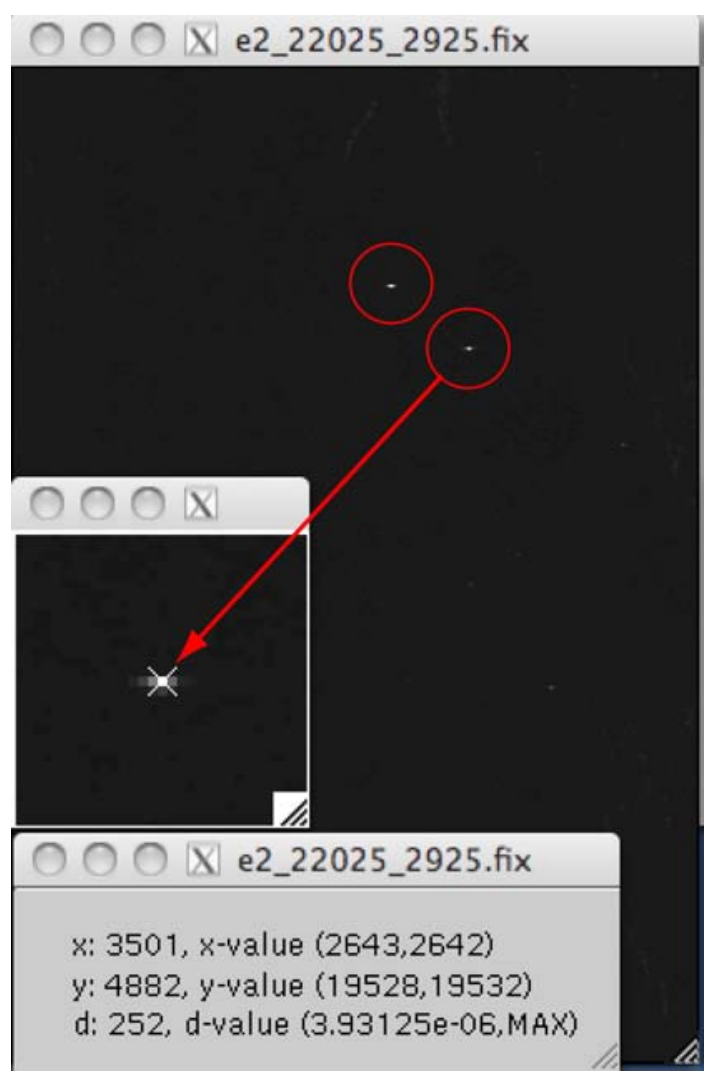


Table E3. Comparison between ENVISAT image and orbit for Pinon corner reflectors D1 and D2 (below).

\begin{tabular}{|l|r|r|r|}
\hline NAME & Doppler & \multicolumn{1}{c|}{ Ri-Ro } & \multicolumn{1}{c|}{ Ai-Ao } \\
\hline ENV1_2_127_2925_07195 & 150.8 & -0.1 & 2.7 \\
\hline ENV1_2_127_2925_07195 & 150.8 & 0.1 & 0.7 \\
\hline ENV1_2_127_2925_12706 & 93.2 & 0.2 & -1.9 \\
\hline ENV1_2_127_2925_12706 & 93.2 & -0.2 & -3.9 \\
\hline ENV1_2_127_2925_13207 & 93.6 & 0.1 & 1.3 \\
\hline ENV1_2_127_2925_13207 & 93.6 & 0.2 & 2.3 \\
\hline ENV1_2_127_2925_38758 & 114.4 & 0.1 & 2.5 \\
\hline ENV1_2_127_2925_38758 & 114.4 & -0.2 & 0.4 \\
\hline ENV1_2_127_2925_44770 & 114.1 & -0.3 & 2.3 \\
\hline ENV1_2_127_2925_44770 & 114.1 & -0.2 & 2.3 \\
\hline mean & & $\mathbf{0 . 0}$ & $\mathbf{0 . 9}$ \\
\hline rms & & $\mathbf{0 . 2}$ & $\mathbf{2 . 2}$ \\
\hline
\end{tabular}

Note a bias of 8.4 in range and 4 in azimuth was added to the orbital range, azimuth co-ordinates.

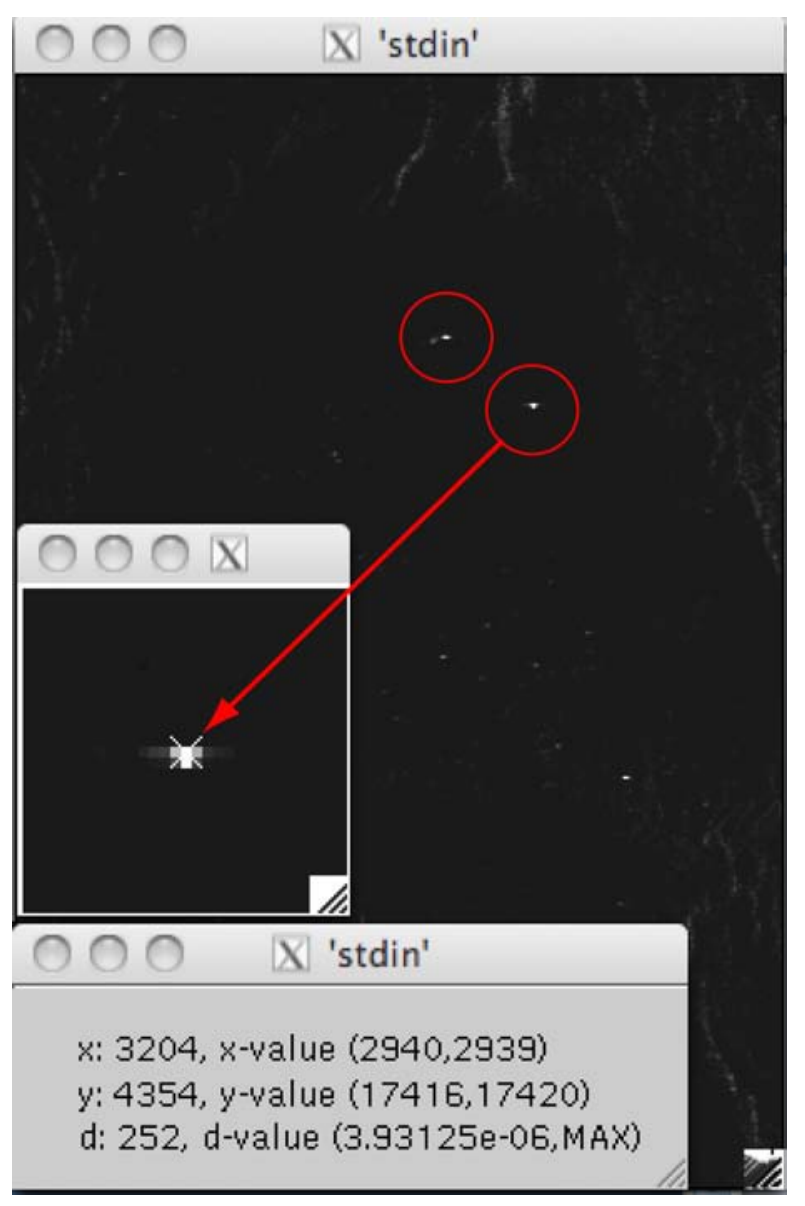


Table E4. Comparison between ALOS image and orbit for Pinon corner reflectors D1 and D2 (right).

\begin{tabular}{|l|r|r|}
\hline NAME & Ri-Ro & \multicolumn{1}{c|}{ Ai-Ao } \\
\hline ALPSRP022200660 & -2.0 & 0.0 \\
\hline ALPSRP028910660 & -2.1 & 0.0 \\
\hline ALPSRP035620660 & -0.1 & 0.8 \\
\hline ALPSRP042330660 & -2.3 & 2.2 \\
\hline ALPSRP049040660 & -1.7 & 0.2 \\
\hline ALPSRP055750660 & -1.9 & 0.8 \\
\hline ALPSRP062460660 & -1.7 & 1.0 \\
\hline ALPSRP075880660 & -0.7 & 2.0 \\
\hline ALPSRP082590660 & -0.6 & 0.0 \\
\hline ALPSRP089300660 & -0.6 & -1.0 \\
\hline ALPSRP096010660 & -1.0 & 1.0 \\
\hline ALPSRP109430660 & -0.9 & 2.0 \\
\hline ALPSRP116140660 & -0.5 & 0.0 \\
\hline ALPSRP122850660 & -0.7 & 2.0 \\
\hline ALPSRP129560660 & -1.1 & 2.0 \\
\hline ALPSRP136270660 & -1.1 & 0.0 \\
\hline ALPSRP142980660 & -1.5 & 0.0 \\
\hline ALPSRP149690660 & -1.7 & -2.0 \\
\hline ALPSRP156400660 & -1.1 & -1.0 \\
\hline ALPSRP163110660 & -0.9 & 0.0 \\
\hline ALPSRP183240660 & -1.3 & 1.0 \\
\hline ALPSRP189950660 & -1.1 & 0.0 \\
\hline ALPSRP196660660 & -1.1 & 0.0 \\
\hline ALPSRP210080660 & -2.5 & 1.0 \\
\hline ALPSRP223500660 & -1.8 & 1.0 \\
\hline ALPSRP230210660 & -0.3 & 2.0 \\
\hline ALPSRP236920660 & -1.0 & 0.0 \\
\hline mean & -1.2 & 0.6 \\
\hline rms & 0.6 & 1.1 \\
\hline
\end{tabular}

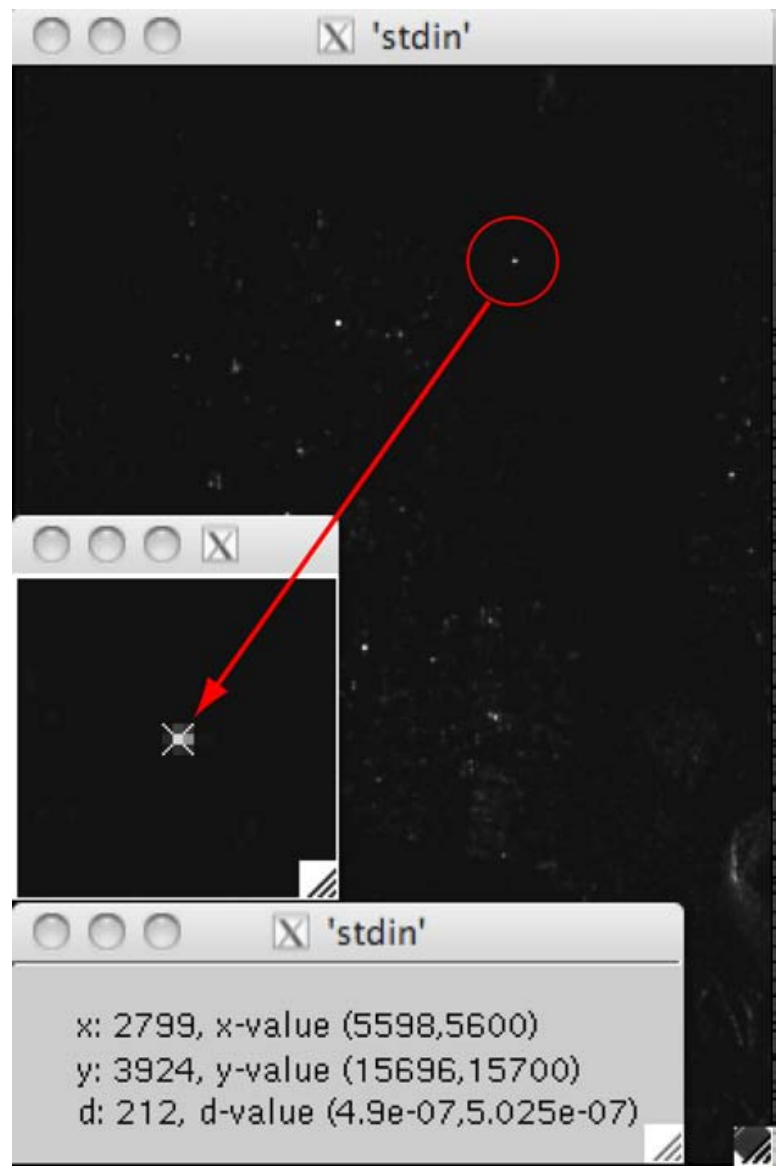


Appendix F - Installation of GMTSAR

GMTSAR has been tested on:

- Mac OS X with powerpc processor

- Mac OS X with i386 processor

- Mac OS X with x86-64 processor

- Linux (Ubuntu) with i686 processor

- Linux with x86-64 processor

- Sun sparc 4

(It should run on any computer with UNIX and a C compiler. The byte order of the machine is irrelevant.)

PREREQUISITES

- C-compiler or Developer tool (Mac)

- TC shell or C shell - do not use bash

A csh script is used to setup environment variables.

Usually your computer will have tcsh or csh installed.

Linux users can use apt-get to install csh or tcsh.

http://www .cyberciti.biz/faq/howto-install-csh-shell-on-linux/

- More than 1.5 GB of memory. The code will run with less memory but could be extremely slow due to paging of memory in esarp.

- GMT and NetCDF (versions GMT4.5.1 and netcdf 3.6.3 or higher)

If you already have the latest GMT and NetCDF working on your computer, ignore STEP 1 (below).

STEP 0. GET GMTSAR SOFTWARE AND SAMPLE DATA

Copy the following two files from the GMTSAR web site http://topex.ucsd.edu/gmtsar

GMTSAR.tar

baja_ALOS.tar.gz (Baja earthquake InSAR data)

(Note this site also has a page to make custom digital elevation models (DEM) to use when processing interferograms for new areas.)

STEP 1. INSTALL GMT AND NETCDF

Go to http://www.soest.hawaii.edu/gmt/ and install GMT and NETCDF.

Alternatively you can use Fink to install GMT and NetCDF. If you use fink you must install gmtshlibs and netcdf-shlibs so the include files and library files will be available. Check to see if GMT is in your path by typing grdinfo or any GMT command. Also you must have the files libgmt.a and libnetcdf.a on your computer. 


\section{STEP 2. UNPACK AND INSTALL GMTSAR}

Put the GMTSAR tar file onto a directory (e.g., /usr/local) and unpack it using tar xfv... Read the README file in GMTSAR/ and follow its instructions to install it.

You will need to modify the environment variables in the gmtsar_config (i.e. GMTHOME, GMTSAR, etc). Note that there are commands in the gmtsar_config where you select the desired cfftld.c code to use. After installation these lines can be commented out. A common error is that the gmtsar_config is not sourced properly. Make sure the command "source /usr/local/GMTSAR/gmtsar_config" is in your .cshrc file. Start a new c-shell to make sure the gmtsar_config is executed and the paths are correct. Type the commands gmtsar.csh, esarp, and grdinfo to make sure things are working.

\section{STEP 3. UNPACK AND PROCESS BAJA EARTHQUAKE DATA}

Put the baja_ALOS.tar.gz onto a proper folder and unpack it with gunzip and tar.

Follow the README.txt in baja_ALOS/

Processing this interferogram usually takes 20-50 min.

FEEDBACK - BAD or GOOD is essential for making improvements

David Sandwell (dsandwell@ucsd.edu)

Rob Mellors (rmellors@geology.sdsu.edu)

Xiaopeng Tong (xitong@ucsd.edu)

Meng Wei (mwei@ucsd.edu)

Paul Wessel (p.wessel@mac.com) 